\title{
Second order parabolic systems, optimal regularity, and singular sets of solutions
}

\section{Systèmes paraboliques de la deuxième ordre, regularité optimale, et des ensembles singuliers des solutions}

\author{
Frank Duzaar $^{\mathrm{a}, *}$, Giuseppe Mingione ${ }^{\mathrm{b}}$ \\ ${ }^{\mathrm{a}}$ Mathematisches Institut der Friedrich-Alexander-Universität, zu Nürnberg-Erlangen, Bismarckstr. 1 1/2, 91054 Erlangen, Germany \\ b Dipartimento di Matematica, Università di Parma, Via D’Azeglio 85/A, 43100 Parma, Italy
}

Received 14 September 2004; accepted 28 October 2004

Available online 10 May 2005

\begin{abstract}
We present a new, complete approach to the partial regularity of solutions to non-linear, second order parabolic systems of the form

$$
u_{t}-\operatorname{div} A(x, t, u, D u)=0 .
$$

In the first part we introduce the $A$-caloric approximation lemma, a parabolic analogue of the harmonic approximation lemma of De Giorgi [Sem. Scuola Normale Superiore Pisa (1960-1961); Lectures in Math., ETH Zürich, Birkhäuser, Basel, 1996] in the version of Simon. This allows to prove optimal partial regularity results for solutions in an elementary way, under minimal and natural assumptions. In the second part we provide estimates for the parabolic Hausdorff dimension of the singular sets of solutions; the proof makes use of parabolic fractional Sobolev spaces.

(C) 2005 L'Association Publications de l'Institut Henri Poincaré. Published by Elsevier B.V. All rights reserved
\end{abstract}

\section{Résumé}

Nous présentons une nouvelle approche complète auprès de la regularité partielle des solutions des systèmes paraboliques, non-linéaires, de la deuxième ordre de la forme

$$
u_{t}-\operatorname{div} A(x, t, u, D u)=0 .
$$

Dans une première partie nous introduisons le lemme d'approximation $A$-calorique, un analogue parabolique du lemme d'approximation harmonique de De Giorgi [Sem. Scuola Normale Superiore Pisa (1960-1961); Lectures in Math., ETH Zürich,

\footnotetext{
* Corresponding author.

E-mail addresses: duzaar@mi.uni-erlangen.de (F. Duzaar), giuseppe.mingione@unipr.it (G. Mingione).
} 
Birkhäuser, Basel, 1996] d'après la version Simon. Cela permet de prouver des résultats optimales de la regularité partielle pour des solutions d'une façon elementaire sous des hypothèses minimales et naturelles. Dans la deuxième partie nous donnons des estimations des ensembles singuliers des solutions pour la dimension parabolique de Hausdorff; la preuve se sert des espaces paraboliques fractionnaires de Sobolev.

(C) 2005 L'Association Publications de l'Institut Henri Poincaré. Published by Elsevier B.V. All rights reserved

Keywords: Partial regularity; Parabolic systems; Singular sets

MSC: 35K55; 35D10

\section{Introduction and results}

In this paper we are concerned with the study of regularity properties of solutions to non-linear, second-order, parabolic systems of the type

$$
u_{t}-\operatorname{div} A(x, t, u, D u)=0, \quad(x, t) \in \Omega \times(-T, 0) \equiv Q_{T},
$$

where $\Omega \subset \mathbb{R}^{n}$ is a bounded domain and $T>0$; for precise notation we refer to the next section.

Our aim is twofold. First we want to explain a new method to prove partial regularity of solutions, that will avoid to assume additional structural assumptions on the system and a priori additional regularity on the solutions. With this new method it is no longer necessary to use various involved tools as Reverse-Hölder inequalities and (parabolic) Gehring's lemma. The method is based on an approximation result that we called the " $A$-caloric approximation lemma", which is explained in Section 3, below. This is the parabolic analogue of the classical harmonic approximation lemma of De Giorgi [7,41] and allows to approximate functions with solutions to parabolic systems with constant coefficients in the same way as the classical harmonic approximation lemma does with harmonic functions. More precisely, in the case of the classical heat system we have (with $B \subset \mathbb{R}^{n}$ denoting the unit ball and $Q:=B \times(-1,0))$

Lemma 1.1 (caloric approximation lemma). For every $\varepsilon>0$ there exists $\delta(n, \varepsilon) \in(0,1)$ with the following property: if $u \in L^{2}\left(-1,0 ; W^{1,2}\left(B, \mathbb{R}^{N}\right)\right)$ with $\int_{Q}\left(|u|^{2}+|D u|^{2}\right) \mathrm{d} z \leqslant 1$ is approximatively caloric in the sense that

$$
\left|\int_{Q}\left(u \varphi_{t}-D u \cdot D \varphi\right) \mathrm{d} z\right| \leqslant \delta \sup _{Q}|D \varphi| \quad \text { for all } \varphi \in C_{0}^{\infty}\left(Q, \mathbb{R}^{N}\right)
$$

then there exists a caloric function $h \in L^{2}\left(-1,0 ; W^{1,2}\left(B, \mathbb{R}^{N}\right)\right)$, i.e. $h_{t}-\Delta h=0$ in $Q$, such that

$$
\int_{Q}\left(|h|^{2}+|D h|^{2}\right) \mathrm{d} z \leqslant 1 \text { and } \int_{Q}|u-h|^{2} \mathrm{~d} z \leqslant \varepsilon .
$$

This lemma and its variant in Section 3 allow to prove partial regularity properties of solutions to non-linear parabolic systems by linearization arguments (see Sections 6 and 7) in a particular efficient and elementary way. In the elliptic setting the possibility of using the harmonic approximation lemma and its non-isotropic variant, the $A$-harmonic approximation lemma, was already exploited both in the setting of Geometric Measure Theory to prove optimal regularity results for minimizing currents to elliptic integrands by Duzaar and Steffen [15] and to prove regularity results for non-linear elliptic systems by Duzaar and Grotowski [11]. In the last case such a method allowed to get an elementary proof of the known regularity results for elliptic systems. These techniques find their origins in Simon's approach, via De Giorgi's harmonic approximation, to Allard's regularity theorem and to the regularity of harmonic maps [40,41]. Later this has been generalized to the degenerate elliptic problems by the authors $[12,13]$. 
In the present paper the use of Lemma 1.1, combined with new ad hoc arguments, allow to prove optimal regularity results for solutions that was not possible to obtain before; it is actually our second aim to provide a complete study of regularity properties of weak solutions: optimal partial regularity exponents and estimates for the dimension of the singular sets. Indeed, the first regularity result of the paper is the following:

Theorem 1.2. Let $u \in L^{2}\left(-T, 0 ; W^{1,2}\left(\Omega, \mathbb{R}^{N}\right)\right)$ be a weak solution to the system (1.1) under the assumptions (2.1)-(2.3) and (2.5) and denote by $Q_{0}$ the set of regular points of $u$ in $Q_{T}$ :

$$
Q_{0}:=\left\{z \in Q_{T}: D u \in C^{\beta, \beta / 2}\left(A, \mathbb{R}^{n N}\right), A\left(\subset Q_{T}\right) \text { is a neighborhood of } z\right\} .
$$

Then $Q_{0}$ is an open subset with full measure and therefore

$$
D u \in C^{\beta, \beta / 2}\left(Q_{0}, \mathbb{R}^{n N}\right), \quad\left|Q_{T} \backslash Q_{0}\right|=0 .
$$

See Section 8 for the proof; of course by $|S|$ we denote the usual Lebesgue measure of a set $S \subset \mathbb{R}^{n+1}$; moreover, $C^{\beta, \beta / 2}(A)$ is the space of functions which are Hölder continuous with exponent $\beta$ with respect to space variable $x$ and with exponent $\beta / 2$ with respect to the time variable $t$; in other words they are Hölder continuous with exponent $\beta$ in the parabolic metric in $\mathbb{R}^{n+1}$ given by

$$
\operatorname{dist}_{p}\left((x, t),\left(x_{0}, t_{0}\right)\right):=\sqrt{\left|x-x_{0}\right|^{2}+\left|t-t_{0}\right|}, \quad x, x_{0} \in \mathbb{R}^{n}, t, t_{0} \in \mathbb{R} .
$$

As far as we are aware, Theorem 1.2 was not known under the general, minimal assumptions considered here. Partial regularity of solutions has been proven for quasi-linear systems [44,17,18,2,25], for non-linear systems with special structure [32] or in low dimensions [37,22,23,34,36], and for non-linear systems only assuming that solutions were a priori more regular [47,29] i.e. bounded or even Hölder continuous; everywhere regularity is possible only under very special (diagonal type) structures, as for instance in the case of the $p$-Laplacian system [9,33], otherwise it fails in general, as shown by counterexamples [45,42,21] and already in the case of elliptic systems $[8,20,46]$. On the other hand, recently, non-a-priori regular solutions have been considered, but again assuming more regularity of $A$ with respect to the "coefficients" $(x, t)$ and, in particular, no dependence on the variable $u[38,1]$; the methods of this last paper are suited for systems with growth conditions more general than the one treated here, but again, they are not suitable to treat the low regularity assumptions we consider. In any case, the optimal regularity stating that the Hölder exponent of the spatial gradient is exactly the same one of the coefficients was never achieved, even under extra assumptions, due to the different techniques available before this paper. We stress here the fact that the use of the "A-caloric approximation lemma", proved in Section 3, allows to get a completely elementary proof of Theorem 1.2, without the use of Reverse-Hölder inequalities; this will be an important point in a forthcoming paper [14], where together with K. Steffen we are going to treat systems with super-linear growth. For such systems higher integrability of solutions has been recently proved by Kinnunen and Lewis [24], and later refined by Misawa [32], but their proof does not yield a Reverse-Hölder inequality since the scaling for parabolic systems of the type in (1.1) with non-linear growth is non-isotropic; therefore the application to partial regularity seems to be not immediately possible, while a further variant of our method will allow to deal with such systems too.

Theorem 1.2 immediately poses a natural problem. Let us call the set of non-regular points "the singular set" of the solution $u$ :

$$
\Sigma:=Q_{T} \backslash Q_{0} .
$$

The natural question is now: how large can $\Sigma$ be? This question can be answered considering the so called parabolic Hausdorff measure in $\mathbb{R}^{n+1}$, that is the canonical Hausdorff measure constructed in $\mathbb{R}^{n+1}$ with respect to the parabolic metric from (1.2). Here we shall consider its cylindrical variant, that leads to slightly stronger estimates. To be precise, let us denote, $x_{0} \in \mathbb{R}^{n}$ and $t_{0} \in \mathbb{R}$

$$
B\left(x_{0}, R\right):=\left\{x \in \mathbb{R}^{n}:\left|x-x_{0}\right|<R\right\}, \quad Q\left(\left(x_{0}, t_{0}\right), R\right):=B\left(x_{0}, R\right) \times\left(t_{0}-R^{2}, t_{0}\right) .
$$


Then we define for $s \in[0, n+2]$ and $F \subset \mathbb{R}^{n+1}$

$$
\mathcal{P}_{s}^{\delta}(F):=\inf \left\{\sum_{i=1}^{\infty} R_{i}^{s}: F \subset \bigcup_{i=1}^{\infty} Q\left(\left(x_{i}, t_{i}\right), R_{i}\right), R_{i} \leqslant \delta\right\}, \quad \mathcal{P}_{S}(F):=\sup _{\delta>0} \mathcal{P}_{s}^{\delta}(F) .
$$

The parabolic Hausdorff dimension is then usually defined according to

$$
\operatorname{dim}_{\mathcal{P}}(F):=\inf \left\{s>0: \mathcal{P}_{S}(F)=0\right\}=\sup \left\{s>0: \mathcal{P}_{S}(F)=\infty\right\} .
$$

Let us observe that due to the stretching in the time direction of the cubes, the limit dimension is $n+2: \operatorname{dim}_{\mathcal{P}}(F) \leqslant$ $n+2$ for every $F \subset \mathbb{R}^{n+1}$, while $\mathcal{P}_{n+2}$ is comparable to the Lebesgue measure in $\mathbb{R}^{n+1}$.

Once again, estimates for the singular set $\Sigma$ have been obtained in very particular situations and when the system in (1.1) shows a simpler structure: $u_{t}-\operatorname{div} A(D u)=0$. In this case it is possible to prove that $\operatorname{dim}_{\mathcal{P}}(\Sigma) \leqslant n[5]$. Actually the problem of proving Hausdorff dimension estimates for systems including Hölder coefficients remained open for a long time already in the elliptic case: $\operatorname{div} A(x, u, D u)=0$ (see the open problems in [16], page 191). It has been finally settled in [31], where it is shown, among other things, that the Hausdorff dimension of solutions to general non-linear elliptic systems in $\mathbb{R}^{n}$ is always strictly less than $n$. Here we shall derive the parabolic analogue of this result, using a difference quotient technique in the setting of parabolic fractional Sobolev spaces; in any case we treat systems with uniformly continuous coefficients, which is a quite standard assumption for partial regularity. Our first result in this direction is the following theorem, whose proof will be presented in Section 9:

Theorem 1.3. Let $u \in L^{2}\left(-T, 0 ; W^{1,2}\left(\Omega, \mathbb{R}^{N}\right)\right)$ be a weak solution to the system (1.1) under the assumptions (2.1)-(2.3) and (2.9) and denote by $\Sigma$ the singular set of $u$. Then there exists a number $\delta \equiv \delta(\beta, L / \lambda)>0$ such that

$$
\operatorname{dim}_{\mathcal{P}}(\Sigma) \leqslant n+2-\delta .
$$

The dependence of $\delta$ upon the regularity of the coefficients $\beta$ and the ellipticity ratio $L / \lambda$ is critical in the sense that

$$
\lim _{\beta \rightarrow 0} \delta=0 \text { and } \lim _{L / \lambda \rightarrow \infty} \delta=0 .
$$

The presence of the small - but quantifiable - number $\delta$ (see Remark 9.6 below), rather than a more consistent quantity, is due to the fact that the vector field $A$ explicitly depends on the function $u(x, t)$, which is a priori only measurable; this yields a strong lack of smoothness for the function

$$
(x, t) \mapsto A(x, t, u(x, t), \cdot),
$$

which prevents the singular set reduction. Indeed, when no dependence on $u$ takes place, the previous result can be substantially improved in the following, which extends previous elliptic results [30]:

Theorem 1.4. Let $u \in L^{2}\left(-T, 0 ; W^{1,2}\left(\Omega, \mathbb{R}^{N}\right)\right)$ be a weak solution to the system

$$
u_{t}-\operatorname{div} A(x, t, D u)=0, \quad(x, t) \in \Omega \times(-T, 0) \equiv Q_{T},
$$

under the assumptions (2.1)-(2.3) and (2.9) and denote by $\Sigma$ the singular set of $u$. Then there exists a number $\delta \equiv \delta(\beta, L / \lambda)>0$ such that

$$
\operatorname{dim}_{\mathcal{P}}(\Sigma) \leqslant n+2-2 \beta-\delta .
$$

The last result, whose proof is in Section 10 , shows that, independently of the ellipticity ratio $L / \lambda$, the singular set dimension depends in a sensitive way on the regularity of the coefficients when considering systems of the type in (1.4). Note that the bound (1.5) is in some sense natural: in the differentiable case $\beta=1$, we find $\operatorname{dim}_{\mathcal{P}}(\Sigma)<n$ 
which agrees (and actually improves) some known estimates for the case $u_{t}-\operatorname{div} A(D u)=0$ [5]. We remark that Theorems 1.3 and 1.4 are the only results where we shall use in a crucial way the a priori higher integrability properties of solutions via the parabolic Gehring's lemma [24].

Finally we point out that most of the previous results, and in particular Theorem 1.2, take place for more general, non-homogeneous systems of the type

$$
u_{t}-\operatorname{div} A(x, t, u, D u)=B(x, t, u, D u), \quad(x, t) \in \Omega \times(-T, 0) \equiv Q_{T},
$$

where the vector field $b$ exhibits critical growth conditions

$$
|B(x, t, u, D u)| \leqslant \tilde{L}\left(1+|D u|^{2}\right),
$$

and the solution is assumed to be bounded with $\|u\|_{\infty}$ satisfying a suitable smallness assumption of the type

$$
2 \tilde{L}\|u\|_{\infty}<L / v \text {. }
$$

We shall not carry out the proofs here, which in many points are even simpler since the solution is assumed to be bounded.

\section{Assumptions and notations}

In the following $\Omega$ will denote a bounded domain in $\mathbb{R}^{n}$, and $Q_{T}$ will denote the parabolic cylinder $\Omega \times(-T, 0)$ where $T>0$. Here we specify the exact assumptions we are going to consider on the parabolic systems. We shall distinguish the assumptions for partial regularity, Theorem 1.2, from the ones for the singular set estimates, Theorems 1.3, 1.4.

Assumptions for Theorem 1.2. We shall consider a vector field $A: Q_{T} \times \mathbb{R}^{N} \times \mathbb{R}^{n N} \rightarrow \mathbb{R}^{n N}$. If $z=(x, t), u \in \mathbb{R}^{N}$ and $p \in \mathbb{R}^{n N}$ we shall denote the coefficients by $A(z, u, p)=A(x, t, u, p)$. We assume that the functions

$$
(z, u, p) \mapsto A(z, u, p), \quad(z, u, p) \mapsto \frac{\partial A}{\partial p}(z, u, p)
$$

are continuous in $Q_{T} \times \mathbb{R}^{N} \times \mathbb{R}^{n N}$ and that the following growth and ellipticity conditions are satisfied:

$$
\begin{aligned}
& |A(z, u, p)| \leqslant L(1+|p|), \\
& \left|\frac{\partial A}{\partial p}(z, u, p)\right| \leqslant L, \\
& \frac{\partial A}{\partial p}(z, u, p) \tilde{p} \cdot \tilde{p} \geqslant \lambda|\tilde{p}|^{2},
\end{aligned}
$$

for all $z \in Q_{T}, u \in \mathbb{R}^{n}$ and $p, \tilde{p} \in \mathbb{R}^{n N}$ where $\lambda>0$ and $1 \leqslant L<\infty$; actually, up to enlarging the constant $L$, (2.1) is a consequence of (2.2); we reported both of them for future convenience. Now we shall specify the regularity assumptions on $A(x, t, u, p)$ with respect to the "coefficients" $(z, u)$; we shall assume that the function

$$
(z, u) \mapsto \frac{A(z, u, p)}{1+|p|}
$$

is Hölder continuous with respect to the parabolic metric (1.2) with Hölder exponent $\beta \in(0,1)$, but not necessarily uniformly Hölder continuous; namely we shall assume that

$$
\left|A(z, u, p)-A\left(z_{0}, u_{0}, p\right)\right| \leqslant L \theta\left(|u|+\left|u_{0}\right|,\left|x-x_{0}\right|+\sqrt{\left|t-t_{0}\right|}+\left|u-u_{0}\right|\right)(1+|p|)
$$

for any $z=(x, t)$ and $z_{0}=\left(x_{0}, t_{0}\right)$ in $Q_{T}, u$ and $u_{0}$ in $\mathbb{R}^{n}$ and for all $p \in \mathbb{R}^{n N}$ where

$$
\theta(y, s):=\min \left\{1, \widetilde{K}(y) s^{\beta}\right\}
$$


and $\widetilde{K}:[0, \infty) \mapsto[1, \infty)$ is a given non-decreasing function. Note that $\theta$ is concave in the second argument. This is the standard way to prescribe (non-uniform) Hölder continuity of the function in (2.4). We find it a bit difficult to handle, therefore, in many points of the paper, we shall use

$$
\left|A(z, u, p)-A\left(z_{0}, u_{0}, p\right)\right| \leqslant K(|u|)\left(\left|x-x_{0}\right|+\sqrt{\left|t-t_{0}\right|}+\left|u-u_{0}\right|\right)^{\beta}(1+|p|)
$$

valid for any $z=(x, t)$ and $z_{0}=\left(x_{0}, t_{0}\right)$ in $Q_{T}, u$ and $u_{0}$ in $\mathbb{R}^{n}$ and for all $p \in \mathbb{R}^{n N}$ where $\beta \in(0,1)$ and $K:[0, \infty) \mapsto[L, \infty)$ is a given non-decreasing function. We note that (2.6) is weaker than (2.5).

Finally we remark a trivial consequence of the continuity of $\partial A / \partial p$; this implies the existence of a function $\omega:[0, \infty) \times[0, \infty) \rightarrow[0, \infty)$ with $\omega(t, 0)=0$ for all $t$ such that $t \mapsto \omega(t, s)$ is nondecreasing for fixed $s, s \mapsto$ $\omega(t, s)^{2}$ is concave and nondecreasing for fixed $t$, and such that

$$
\left|\frac{\partial A}{\partial p}(x, t, u, p)-\frac{\partial A}{\partial p}\left(x_{0}, t_{0}, u_{0}, p_{0}\right)\right| \leqslant \omega\left(M,\left|x-x_{0}\right|^{2}+\left|t-t_{0}\right|+\left|u-u_{0}\right|^{2}+\left|p-p_{0}\right|^{2}\right)
$$

for any $z=(x, t)$ and $z_{0}=\left(x_{0}, t_{0}\right)$ in $Q_{T}$, any $u, u_{0}$ in $\mathbb{R}^{n}$ and $p, p_{0} \in \mathbb{R}^{n N}$ whenever $|u|+|p|+\left|u-u_{0}\right|+$ $\left|p-p_{0}\right| \leqslant M$.

Remark 2.1. In the case of systems of the type (1.4) both (2.5) and (2.6) must be replaced by

$$
\left|A(z, p)-A\left(z_{0}, p\right)\right| \leqslant L\left(\left|x-x_{0}\right|+\sqrt{\left|t-t_{0}\right|}\right)^{\beta}(1+|p|),
$$

while, in order to apply (2.7), we just need to require that $|p|+\left|p-p_{0}\right| \leqslant M$.

Additional assumptions for Theorems 1.3 and 1.4. In order to prove Theorems 1.3 and 1.4 we shall be forced to consider systems with uniformly Hölder continuous coefficients: we shall consider the following reinforcement of (2.6):

$$
\left|A(z, u, p)-A\left(z_{0}, u_{0}, p\right)\right| \leqslant L \omega\left(\left|x-x_{0}\right|+\sqrt{\left|t-t_{0}\right|}+\left|u-u_{0}\right|\right)(1+|p|)
$$

where $\omega:[0, \infty) \mapsto[0,1]$ is a continuous concave function such that

$$
\omega(s) \leqslant s^{\beta}, \quad s>0 .
$$

Finally we recall that a weak solution to the system (1.1) is a function $u \in L^{2}\left(-T, 0 ; W^{1,2}\left(\Omega, \mathbb{R}^{N}\right)\right)$ such that

$$
\int_{Q_{T}}\left(u \varphi_{t}-A(z, u, D u) D \varphi\right) \mathrm{d} z=0, \quad \text { for all } \varphi \in C_{0}^{\infty}\left(Q_{T}, \mathbb{R}^{N}\right) .
$$

\section{Preliminaries}

If $v$ is an integrable function in $Q\left(z_{0}, \varrho\right) \equiv Q_{\varrho}\left(z_{0}\right)=B_{\varrho}\left(x_{0}\right) \times\left(t_{0}-\varrho^{2}, t_{0}\right), z_{0}=\left(x_{0}, t_{0}\right)$, we will denote its average by

$$
(v)_{z_{0}, \varrho}:=\int_{Q_{\varrho}\left(z_{0}\right)} v \mathrm{~d} z:=\frac{1}{\alpha_{n} \varrho^{n+2}} \int_{Q_{\varrho}\left(z_{0}\right)} v \mathrm{~d} z
$$

where $\alpha_{n}$ denotes the volume of the unit ball in $\mathbb{R}^{n}$. We remark that in the following, when not crucial, the "center" of the cylinder will be often unspecified e.g. $Q_{\varrho}\left(z_{0}\right) \equiv Q_{\varrho}$; the same convention will be adopted for balls in $\mathbb{R}^{n}$ thereby denoting $B\left(x_{0}, \varrho\right) \equiv B_{\varrho}\left(x_{0}\right)$. Finally in the rest of the paper the symbol $c$ will denote a positive, finite constant that may vary from line to line; the relevant dependencies will be specified. 
For $u \in L^{2}\left(Q_{\varrho}\left(z_{0}\right), \mathbb{R}^{N}\right)$ we denote by $\ell_{z_{0}, \varrho}$ the unique affine function (in space) $\ell(z)=\ell(x)$ minimizing

$$
\ell \mapsto \int_{Q_{\varrho}\left(z_{0}\right)}|u-\ell|^{2} \mathrm{~d} z
$$

amongst all affine functions $a(z)=a(x)$ which are independent of $t$. To get an explicit formula for $\ell_{z_{0}, \varrho}$ we note that such a unique minimum point exists and takes the form

$$
\ell_{z_{0}, \varrho}(x)=\xi_{z_{0}, \varrho}+v_{z_{0}, \varrho}\left(x-x_{0}\right),
$$

where $v_{z_{0}, \varrho} \in \mathbb{R}^{n N}$. A straightforward computation yields that

$$
f_{Q_{\varrho}\left(z_{0}\right)} u \cdot a(x) \mathrm{d} z=f_{Q_{\varrho}\left(z_{0}\right)} \ell_{z_{0}, \varrho}(x) \cdot a(x) \mathrm{d} z
$$

for any affine function $a(x)=\xi+v\left(x-x_{0}\right)$ with $\xi \in \mathbb{R}^{N}$ and $v \in \mathbb{R}^{n N}$. This implies in particular that

$$
\xi_{z_{0}, \varrho}=f_{Q_{\varrho}\left(z_{0}\right)} u \mathrm{~d} z=(u)_{z_{0}, \varrho} \quad \text { and } \quad \nu_{z_{0}, \varrho}=\frac{n+2}{\varrho^{2}} f_{Q_{\varrho}\left(z_{0}\right)} u \otimes\left(x-x_{0}\right) \mathrm{d} z .
$$

For convenience of the reader we recall from [26] the following:

Lemma 3.1. Let $u \in L^{2}\left(Q_{\varrho}\left(z_{0}\right), \mathbb{R}^{N}\right), 0<\vartheta<1$, and $\ell_{z_{0}, \varrho}$ respectively $\ell_{z_{0}, \vartheta \varrho}$ the unique affine functions minimizing $\ell \mapsto f_{Q_{\varrho}\left(z_{0}\right)}|u-\ell|^{2} \mathrm{~d} z$ respectively $\ell \mapsto f_{Q_{\vartheta_{\varrho}}\left(z_{0}\right)}|u-\ell|^{2} \mathrm{~d} z$. Then there holds

$$
\left|v_{z_{0}, \vartheta \varrho}-v_{z_{0}, \varrho}\right|^{2} \leqslant \frac{n(n+2)}{(\vartheta \varrho)^{2}} f_{Q_{\vartheta \varrho}\left(z_{0}\right)}\left|u-(u)_{z_{0}, \varrho}-v_{z_{0}, \varrho}\left(x-x_{0}\right)\right|^{2} \mathrm{~d} z .
$$

Moreover, if $D u \in L^{2}\left(Q_{\varrho}\left(z_{0}\right), \mathbb{R}^{n N}\right)$ we have

$$
\left|v_{z_{0}, \varrho}-(D u)_{z_{0}, \varrho}\right|^{2} \leqslant \frac{n(n+2)}{\varrho^{2}} f_{Q_{\varrho}\left(z_{0}\right)}\left|u-(u)_{z_{0}, \varrho}-(D u)_{z_{0}, \varrho}\left(x-x_{0}\right)\right|^{2} \mathrm{~d} z .
$$

The following lemma is a (parabolic variant) of a well known measure theoretical result; the proof can be obtained along the lines of [19], Chapter 3 and [30], Section 4.

Lemma 3.2. Let $\mu: \mathcal{B}_{1} \times \mathcal{B}_{n} \rightarrow \mathbb{R}^{+}$be a bounded, increasing set-function (here $\mathcal{B}_{1}, \mathcal{B}_{n}$ denote the families of Borel subsets of $(-T, 0)$ and $\Omega$, respectively), which also satisfies

$$
\sum_{i \in \mathbb{N}} \mu\left(Q_{R_{i}}\left(z_{i}\right)\right) \leqslant \mu(\Omega \times(-T, 0)),
$$

whenever $\left\{Q_{R_{i}}\left(z_{i}\right)\right\}_{i \in \mathbb{N}}$ is a family of pairwise disjoint parabolic cylinders in $\Omega \times(-T, 0)$. If we let

$$
A:=\left\{z_{0} \in \Omega \times(-T, 0): \liminf _{\varrho \downarrow 0} \varrho^{-s} \mu\left(Q_{\varrho}\left(z_{0}\right)\right)>0\right\}, \quad 0<s \leqslant n+2,
$$

then

$$
\operatorname{dim}_{\mathcal{P}}(A) \leqslant s .
$$


Now we recall the definition of the parabolic fractional Sobolev spaces for which we refer to [27]. We shall say that a function $u \in L^{2}\left(Q_{T}, \mathbb{R}^{k}\right)$ belongs to the fractional Sobolev space $W^{\alpha, \theta ; 2}\left(Q_{T}, \mathbb{R}^{k}\right), \alpha, \theta \in(0,1), k \in \mathbb{N}$, provided

$$
\iint_{-T}^{0} \int_{\Omega} \frac{|u(x, t)-u(y, t)|^{2}}{|x-y|^{n+2 \alpha}} \mathrm{d} x \mathrm{~d} y \mathrm{~d} t+\iint_{\Omega} \int_{-T}^{0} \int_{-T}^{0} \frac{|u(x, t)-u(x, s)|^{2}}{|t-s|^{1+2 \theta}} \mathrm{d} t \mathrm{~d} s \mathrm{~d} x=:[u]_{\alpha, \theta ; Q_{T}}<\infty .
$$

The local variant $W_{\text {loc }}^{\alpha, \theta ; 2}\left(Q_{T}, \mathbb{R}^{k}\right)$ can be defined in the usual way; it is also possible to define spaces $W^{\alpha, \theta ; 2}\left(Q_{T}, \mathbb{R}^{k}\right)$ for higher values of $\alpha$ and $\theta$; for this we refer to [27]. The following Poincaré type inequality can then be obtained in a standard way (for instance imitating the proof of Proposition 3.1 from [10]):

$$
\int_{Q_{\varrho}\left(z_{0}\right)}\left|u(z)-(u)_{z_{0}, \varrho}\right|^{2} \mathrm{~d} z \leqslant c(n) \varrho^{2 \beta}[u]_{\beta, \beta / 2 ; Q_{\varrho}\left(z_{0}\right)}, \quad \beta \in(0,1),
$$

for any function $u \in W^{\beta, \beta / 2 ; 2}\left(Q_{\varrho}, \mathbb{R}^{k}\right)$.

Proposition 3.3. Let $u \in W_{\text {loc }}^{\beta, \beta / 2 ; 2}\left(Q_{T}, \mathbb{R}^{k}\right)$ for $\beta>0$ and let

$$
\begin{aligned}
& A:=\left\{z_{0} \in Q_{T}: \liminf _{\varrho \downarrow 0} \underset{Q_{\varrho}\left(z_{0}\right)}{f}\left|u-(u)_{z_{0}, \varrho}\right|^{2} \mathrm{~d} z>0\right\}, \\
& B:=\left\{z_{0} \in Q_{T}: \underset{\varrho \downarrow 0}{\limsup }\left|(u)_{z_{0}, \varrho}\right|=\infty\right\} .
\end{aligned}
$$

Then

$$
\operatorname{dim}_{\mathcal{P}}(A) \leqslant n+2-2 \beta, \quad \operatorname{dim}_{\mathcal{P}}(B) \leqslant n+2-2 \beta .
$$

The proof can be obtained along the lines of the analogue, classical results for standard fractional Sobolev functions (see for instance [10,30] for elementary proofs). We briefly sketch the main arguments, confining ourselves to the estimate for $A$. By a standard localization argument [30] we may suppose without loss of generality that $u \in W^{\beta, \beta / 2 ; 2}\left(Q_{T}, \mathbb{R}^{k}\right)$; then by (3.1) we have that $A \subset S$ where

$$
S:=\left\{z_{0} \in Q_{T}: \liminf _{\varrho \downarrow 0} \varrho^{-(n+2)+2 \beta}[u]_{\beta, \beta / 2 ; Q_{\varrho}\left(z_{0}\right)}>0\right\} .
$$

Therefore, applying Lemma 3.2 we have $\operatorname{dim}_{\mathcal{P}}(A) \leqslant \operatorname{dim}_{\mathcal{P}}(S) \leqslant n+2-2 \beta$; we observe that the application of Lemma 3.2 is possible since the set function

$$
\mu: I \times \omega \rightarrow \iint_{I} \int_{\omega} \frac{|u(x, t)-u(y, t)|^{2}}{|x-y|^{n+2 \beta}} \mathrm{d} x \mathrm{~d} y \mathrm{~d} t+\iint_{\omega} \int_{I} \frac{|u(x, t)-u(x, s)|^{2}}{|t-s|^{1+\beta}} \mathrm{d} t \mathrm{~d} s \mathrm{~d} x,
$$

with $I \subset(-T, 0)$ and $\omega \subset \Omega$, Borel sets, clearly meets all the requirements of Lemma 3.2.

Finally we conclude with the parabolic version of the well known relation between Nikolski spaces and Fractional Sobolev spaces; the proof can be obtained by a straightforward adaptation of the standard elliptic result $[30,10]$.

Proposition 3.4. Let $u \in L^{2}\left(Q_{T}, \mathbb{R}^{k}\right)$. Suppose that

$$
\int_{\widetilde{Q}}|u(x, t+h)-u(x, t)|^{2} \mathrm{~d} x \mathrm{~d} t \leqslant c_{1}|h|^{2 \theta}, \quad \theta \in(0,1),
$$


where $\widetilde{Q}:=\widetilde{\Omega} \times(-T+d,-d)$ and $\widetilde{\Omega} \Subset \Omega$, for every $h \in \mathbb{R}$ such that $|h| \leqslant \min \{d, A\}$, where $d \in(0, T / 8), A>0$ are positive constants. Then there exists a constant $\tilde{c}_{1} \equiv \tilde{c}_{1}\left(\theta, \gamma, d, A, c_{1},\|u\|_{L^{2}\left(Q_{T}\right)}\right)>0$ such that

$$
\int_{\widetilde{\Omega}} \int_{-T+d}^{-d} \int_{-T+d}^{-d} \frac{|u(x, t)-u(x, s)|^{2}}{|t-s|^{1+2 \gamma}} \mathrm{d} t \mathrm{~d} s \mathrm{~d} x \leqslant \tilde{c}_{1}, \quad \forall \gamma \in(0, \theta) .
$$

Suppose that

$$
\int_{\widetilde{Q}}\left|u\left(x+h e_{s}, t\right)-u(x, t)\right|^{2} \mathrm{~d} x \mathrm{~d} t \leqslant c_{2}|h|^{2 \theta}, \quad \theta \in(0,1),
$$

for every $|h| \leqslant \min \{\operatorname{dist}(\widetilde{\Omega}, \partial \Omega), A\}, \forall s \in\{1, \ldots, n\}$, where $\left\{e_{s}\right\}_{1 \leqslant s \leqslant n}$ is the standard basis of $\mathbb{R}^{n}$. Then for every $\mathcal{O} \Subset \widetilde{\Omega}$ there exists a constant $\tilde{c}_{2} \equiv \tilde{c}_{2}\left(n, \theta, \gamma, A, c_{2}, \operatorname{dist}(\widetilde{\Omega}, \partial \Omega), \operatorname{dist}(\mathcal{O}, \partial \widetilde{\Omega}),\|u\|_{L^{2}\left(Q_{T}\right)}\right)>0$ such that

$$
\int_{-T+d}^{-d} \int_{\mathcal{O}} \int_{\mathcal{O}} \frac{|u(x, t)-u(y, t)|^{2}}{|x-y|^{n+2 \gamma}} \mathrm{d} x \mathrm{~d} y \mathrm{~d} t \leqslant \tilde{c}_{2}, \quad \forall \gamma \in(0, \theta) .
$$

\section{A-caloric approximation}

We recall that a strongly elliptic bilinear form $A$ on $\mathbb{R}^{n N}$ with ellipticity constant $\lambda>0$ and upper bound $\Lambda>0$ means that

$$
\lambda|\tilde{p}|^{2} \leqslant A(\tilde{p}, \tilde{p}), \quad A(p, \tilde{p}) \leqslant \Lambda|p||\tilde{p}| \quad \forall p, \tilde{p} \in \mathbb{R}^{n N} .
$$

We shall say that a function $h \in L^{2}\left(-1,0 ; W^{1,2}\left(B_{\varrho}, \mathbb{R}^{N}\right)\right)$ is $A$-caloric on $Q_{\varrho}$ if it satisfies

$$
\int_{Q_{\varrho}}\left(h \varphi_{t}-A(D h, D \varphi)\right) \mathrm{d} z=0 \quad \text { for all } \varphi \in C_{0}^{\infty}\left(Q_{\varrho}, \mathbb{R}^{N}\right) .
$$

Obviously, when $A(\tilde{p}, \tilde{p}) \equiv|\tilde{p}|^{2}$ for every $\tilde{p} \in \mathbb{R}^{n N}$, then an $A$-caloric function is just a caloric function

$$
h_{t}-\Delta h=0 \text {, }
$$

and therefore Lemma 1.1 is just a particular case of the following:

Lemma 4.1 (A-caloric approximation lemma). There exists a positive function $\delta(n, N, \lambda, \Lambda, \varepsilon) \leqslant 1$ with the following property: Whenever $A$ is a bilinear form on $\mathbb{R}^{n N}$ which is strongly elliptic with ellipticity constant $\lambda>0$ and upper bound $\Lambda, \varepsilon$ is a positive number, and $u \in L^{2}\left(-1,0 ; W^{1,2}\left(B, \mathbb{R}^{N}\right)\right)$ with

$$
\int_{Q}\left(|u|^{2}+|D u|^{2}\right) \mathrm{d} z \leqslant 1
$$

is approximatively A-caloric in the sense that

$$
\left|\int_{Q}\left(u \varphi_{t}-A(D u, D \varphi)\right) \mathrm{d} z\right| \leqslant \delta \sup _{Q}|D \varphi| \text { for all } \varphi \in C_{0}^{\infty}\left(Q, \mathbb{R}^{N}\right)
$$

then there exists an A-caloric function $h$ such that

$$
\int_{Q}\left(|h|^{2}+|D h|^{2}\right) \mathrm{d} z \leqslant 1 \text { and } \int_{Q}|u-h|^{2} \mathrm{~d} z \leqslant \varepsilon .
$$


Proof. Were the assertion false, we could find $\varepsilon>0$, a sequence $\left(A_{k}\right)$ of bilinear forms on $\mathbb{R}^{n N}$, with uniform ellipticity bound $\lambda>0$ and uniform upper bound $\Lambda$, and a sequence of functions $\left(v_{k}\right)_{k \in \mathbb{N}}$ with $v_{k} \in$ $L^{2}\left(-1,0 ; W^{1,2}\left(B, \mathbb{R}^{N}\right)\right)$, such that

$$
\int_{Q}\left(\left|v_{k}\right|^{2}+\left|D v_{k}\right|^{2}\right) \mathrm{d} z \leqslant 1
$$

and

$$
\left|\int_{Q}\left(v_{k} \varphi_{t}-A_{k}\left(D v_{k}, D \varphi\right)\right) \mathrm{d} z\right| \leqslant \frac{1}{k} \sup _{Q}|D \varphi|
$$

for all $\varphi \in C_{0}^{1}\left(B, \mathbb{R}^{N}\right)$ and $k \in \mathbb{N}$, but

$$
\int_{Q}\left|v_{k}-h\right|^{2} \mathrm{~d} z>\varepsilon \quad \text { for all } h \in \mathcal{H}_{k},
$$

where here

$$
\mathcal{H}_{k}=\left\{f \in L^{2}\left(-1,0 ; W^{1,2}\left(B, \mathbb{R}^{N}\right)\right): f \text { is an } A_{k} \text {-caloric function on } Q, \int_{Q}\left(|f|^{2}+|D f|^{2}\right) \mathrm{d} z \leqslant 1\right\} .
$$

Passing to a subsequence (also labeled with $k$ ) we obtain the existence of $v \in L^{2}\left(-1,0 ; W^{1,2}\left(B, \mathbb{R}^{N}\right)\right.$ ) and $A$ such that there holds

$$
\begin{cases}v_{k} \rightarrow v & \text { weakly in } L^{2}\left(Q, \mathbb{R}^{N}\right) \\ D v_{k} \rightarrow D v & \text { weakly in } L^{2}\left(Q, \mathbb{R}^{n N}\right) \\ A_{k} \rightarrow A & \text { as bilinear forms on } \mathbb{R}^{n N}\end{cases}
$$

Using the lower semicontinuity of $v \mapsto \int_{Q}\left(|v|^{2}+|D v|^{2}\right) \mathrm{d} z$ with respect to weak convergence in $L^{2}(-1,0$; $\left.W^{1,2}\left(B, \mathbb{R}^{N}\right)\right)$ we obtain

$$
\int_{Q}\left(|v|^{2}+|D v|^{2} \mathrm{~d} z\right) \leqslant 1 .
$$

Moreover, for $\varphi \in C_{0}^{\infty}\left(Q, \mathbb{R}^{N}\right)$ we have

$$
\begin{aligned}
& \int_{Q}\left(v \varphi_{t}-A(D v, D \varphi)\right) \mathrm{d} z \\
& \quad=\int_{Q}\left(\left(v-v_{k}\right) \varphi_{t}-A\left(D v-D v_{k}, D \varphi\right)\right) \mathrm{d} z-\int_{Q}\left(A-A_{k}\right)\left(D v_{k}, D \varphi\right) \mathrm{d} z+\int_{Q}\left(v_{k} \varphi_{t}-A_{k}\left(D v_{k}, D \varphi\right)\right) \mathrm{d} z .
\end{aligned}
$$

Passing to the limit $k \rightarrow \infty$ we see that the first term of the right-hand side converges to 0 due to (4.4); the same holds for the second term in view of the uniform bound of $D v_{k}$ in $L^{2}\left(Q, \mathbb{R}^{n N}\right)$ (see (4.1)) and the convergence of the $A_{k}$ 's; the third term vanishes in the limit $k \rightarrow \infty$ via (4.2). This shows that the weak limit $v$ is an $A$-caloric function on $Q$, i.e.

$$
\int_{Q}\left(v \varphi_{t}-A(D v, D \varphi)\right) \mathrm{d} z=0, \quad \forall \varphi \in C_{0}^{\infty}\left(Q, \mathbb{R}^{N}\right) .
$$


Now, in order to get compactness in $L^{2}\left(Q, \mathbb{R}^{N}\right)$, i.e. $v_{k} \rightarrow v$ in $L^{2}\left(Q, \mathbb{R}^{N}\right)$, we estimate the time derivatives of $v_{k}$. For this we let $\varphi \in C_{0}^{\infty}\left(Q, \mathbb{R}^{N}\right)$ and compute $(z=(x, t)$ and $Q=B \times(-1,0))$ :

$$
\begin{aligned}
\left|\int_{Q} v_{k} \varphi_{t} \mathrm{~d} z\right| & \leqslant\left|\int_{-1}^{0} \int_{B} A_{k}\left(D v_{k}, D \varphi\right) \mathrm{d} x \mathrm{~d} t\right|+\frac{1}{k} \sup _{-1 \leqslant t \leqslant 0}\|D \varphi(\cdot, t)\|_{L^{\infty}(B)} \\
& \leqslant\left|A_{k}\right| \int_{-1}^{0}\left\|D v_{k}(\cdot, t)\right\|_{L^{2}(B)}\|D \varphi(\cdot, t)\|_{L^{2}(B)} \mathrm{d} t+\frac{1}{k} \sup _{-1 \leqslant t \leqslant 0}\|D \varphi(\cdot, t)\|_{L^{\infty}(B)} \\
& \leqslant\left|A_{k}\right|\left(\int_{-1}^{0}\left\|D v_{k}(\cdot, t)\right\|_{L^{2}(B)}^{2} \mathrm{~d} t\right)^{1 / 2}\left(\int_{-1}^{0}\|D \varphi(\cdot, t)\|_{L^{2}(B)}^{2} \mathrm{~d} t\right)^{1 / 2}+\frac{1}{k} \sup _{-1 \leqslant t \leqslant 0}\|D \varphi(\cdot, t)\|_{L^{\infty}(B)} \\
& \leqslant\left|A_{k}\right| \int_{-1}^{0}\|D \varphi(\cdot, t)\|_{L^{2}(B)}^{2} \mathrm{~d} t+\frac{1}{k} \sup _{-1 \leqslant t \leqslant 0}\|D \varphi\|_{L^{\infty}(B)} .
\end{aligned}
$$

Here we have used in turn (4.2), the Cauchy-Schwartz inequality and (4.1). Now, for $-1<s_{1}<s_{2}<0$ and $\varepsilon>0$ small enough we choose

$$
\zeta_{\varepsilon}(t)= \begin{cases}0, & \text { for }-1 \leqslant t \leqslant s_{1}-\varepsilon, \\ \frac{1}{\varepsilon}\left(t-s_{1}+\varepsilon\right) & \text { for } s_{1}-\varepsilon \leqslant t \leqslant s_{1}, \\ 1 & \text { for } s_{1} \leqslant t \leqslant s_{2}, \\ -\frac{1}{\varepsilon}\left(t-s_{2}-\varepsilon\right) & \text { for } s_{2} \leqslant t \leqslant s_{2}+\varepsilon, \\ 0 & \text { for } s_{2}+\varepsilon \leqslant t \leqslant 1,\end{cases}
$$

and let $\varphi(x, t)=\zeta_{\varepsilon}(t) \psi(x)$ for $\psi \in C_{0}^{\infty}\left(B, \mathbb{R}^{N}\right)$. Testing (4.6) with $\varphi$ we obtain

$$
\begin{aligned}
& \left|\int_{B}\left(\frac{1}{\varepsilon} \int_{s_{1}-\varepsilon}^{s_{1}} v_{k}(x, t) \mathrm{d} t-\frac{1}{\varepsilon} \int_{s_{2}}^{s_{2}+\varepsilon} v_{k}(x, t) \mathrm{d} t\right) \cdot \psi(x) \mathrm{d} x\right| \\
& \quad \leqslant\left|A_{k}\right|\left(\int_{-1}^{0} \zeta_{\varepsilon}(t)^{2} \mathrm{~d} t\right)^{1 / 2}\|D \psi\|_{L^{2}(B)}+\frac{1}{k}\|D \psi\|_{L^{\infty}(B)} \sup _{-1 \leqslant t \leqslant 0} \zeta_{\varepsilon}(t) \\
& \quad \leqslant\left(\left|A_{k}\right| \sqrt{s_{2}-s_{1}+2 \varepsilon}+\frac{1}{k}\right)\|D \psi\|_{L^{\infty}(B)} .
\end{aligned}
$$

By Sobolev-embedding

$$
\|D \psi\|_{L^{\infty}(B)} \leqslant c(n, \ell)\|\psi\|_{W_{0}^{\ell, 2}(B)}, \quad \ell>\frac{n+2}{2},
$$

we see that

$$
\begin{aligned}
& \left|\int_{B}\left(\frac{1}{\varepsilon} \int_{s_{1}-\varepsilon}^{s_{1}} v_{k}(x, t) \mathrm{d} t-\frac{1}{\varepsilon} \int_{s_{2}}^{s_{2}+\varepsilon} v_{k}(x, t) \mathrm{d} t\right) \cdot \psi(x) \mathrm{d} x\right| \\
& \quad \leqslant c(n, \ell)\left(\left|A_{k}\right| \sqrt{s_{2}-s_{1}+2 \varepsilon}+\frac{1}{k}\right)\|\psi\|_{W_{0}^{\ell, 2}(B)} .
\end{aligned}
$$


Passing to the limit $\varepsilon \downarrow 0$ we obtain for a.e. $-1<s_{1}<s_{2}<0$

$$
\left|\int_{B}\left(v_{k}\left(\cdot, s_{2}\right)-v_{k}\left(\cdot, s_{1}\right)\right) \cdot \psi \mathrm{d} x\right| \leqslant c(n, \ell)\left(\left|A_{k}\right| \sqrt{s_{2}-s_{1}}+\frac{1}{k}\right)\|\psi\|_{W_{0}^{\ell, 2}(B)}
$$

for any $\psi \in C_{0}^{\infty}\left(B, \mathbb{R}^{N}\right)$. By density of $C_{0}^{\infty}\left(B, \mathbb{R}^{N}\right)$ in $W_{0}^{\ell, 2}\left(B, \mathbb{R}^{N}\right)$ the last inequality is also valid for any $\psi \in W_{0}^{\ell, 2}\left(B, \mathbb{R}^{N}\right)$. Taking the supremum over all $\psi \in W_{0}^{\ell, 2}\left(B, \mathbb{R}^{N}\right)$ with $\|\psi\|_{W_{0}^{\ell, 2}(B)} \leqslant 1$ we infer

$$
\left\|v_{k}\left(\cdot, s_{2}\right)-v_{k}\left(\cdot, s_{1}\right)\right\|_{W^{-\ell, 2}\left(B, \mathbb{R}^{N}\right)} \leqslant c(\ell, n)\left(\left|A_{k}\right| \sqrt{s_{2}-s_{1}}+\frac{1}{k}\right) .
$$

Interpolating $L^{2}\left(B, \mathbb{R}^{N}\right)$ between $W^{1,2}\left(B, \mathbb{R}^{N}\right)$ and $W^{-\ell, 2}\left(B, \mathbb{R}^{N}\right)$ it follows for $\mu>0$ that

$$
\begin{aligned}
& \int_{-1}^{-h}\left\|v_{k}(\cdot, t+h)-v_{k}(\cdot, t)\right\|_{L^{2}(B)}^{2} \mathrm{~d} t \\
& \leqslant \mu \int_{-1}^{-h}\left\|v_{k}(\cdot, t+h)-v_{k}(\cdot, t)\right\|_{W^{1,2}(B)}^{2} \mathrm{~d} t+c(\mu) \int_{-1}^{-h}\left\|v_{k}(\cdot, t+h)-v_{k}(\cdot, t)\right\|_{W^{-\ell, 2}(B)}^{2} \mathrm{~d} t \\
& \leqslant 4 \mu \int_{-1}^{0}\left\|v_{k}(\cdot, t)\right\|_{W^{1,2}(B)}^{2} \mathrm{~d} t+c(\mu) c^{2}\left(\left|A_{k}\right| \sqrt{h}+\frac{1}{k}\right)^{2} \leqslant 4 \mu+2 c(\mu) c^{2}\left(\left|A_{k}\right|^{2} h+\frac{1}{k^{2}}\right) .
\end{aligned}
$$

Here, we have used in the first line the interpolation inequality

$$
\|w\|_{L^{2}(B)}^{2} \leqslant \mu\|w\|_{W^{1,2}(B)}^{2}+c(\mu)\|w\|_{W^{-\ell, 2}(B)}^{2}
$$

valid for $w \in W^{1,2}\left(B, \mathbb{R}^{N}\right)$. Moreover, in the second-last line we have used the bound (4.7) for $\| v_{k}(\cdot, t+h)-$ $v_{k}(\cdot, t) \|_{W^{-\ell, 2(B)}}$ from above and the bound (4.1), i.e. $\int_{Q}\left(\left|v_{k}\right|^{2}+\left|D v_{k}\right|^{2}\right) \mathrm{d} z \leqslant 1$.

We are now in the position to show that

$$
\lim _{h \downarrow 0} \int_{-1}^{-h}\left\|v_{k}(\cdot, t+h)-v_{k}(\cdot, t)\right\|_{L^{2}(B)}^{2} \mathrm{~d} t=0 \quad \text { uniformly in } k .
$$

In order to do this we recall that $A_{k} \rightarrow A$ as $k \rightarrow \infty$ so that $\sup _{k \geqslant 1}\left|A_{k}\right| \leqslant a<\infty$. Using this in (4.8) we obtain

$$
\int_{-1}^{-h}\left\|v_{k}(\cdot, t+h)-v_{k}(\cdot, t)\right\|_{L^{2}(B)}^{2} \mathrm{~d} t \leqslant 4 \mu+2 c(\mu) c^{2}\left(a^{2} h+\frac{1}{k^{2}}\right) .
$$

For given $\theta>0$ we choose $\mu=\frac{\theta}{12}$. This fixes $\mu$ and also $c(\mu)=c\left(\frac{1}{12} \theta\right)$. Next we choose $k_{0} \in \mathbb{N}$ such that $\frac{2 c(\mu) c^{2}}{k^{2}}<\frac{\theta}{3}$ for any $k \geqslant k_{0}$. Then, for $k=1, \ldots, k_{0}-1$ we choose $h_{1}>0$ such that

$$
\int_{-1}^{-h}\left\|v_{k}(\cdot, t+h)-v_{k}(\cdot, t)\right\|_{L^{2}(B)}^{2} \mathrm{~d} t<\theta \quad \forall 0<h<h_{1}, k=1, \ldots, k_{0}-1 .
$$


Finally, we choose $h_{2}>0$ such that $2 c(\mu) c^{2} a^{2} h<\frac{\theta}{3}$ for any $0<h<h_{2}$. Then, for any $k \in \mathbb{N}$ and $0<h<h_{0}:=$ $\min \left(h_{1}, h_{2}\right)$ we have

$$
\int_{-1}^{-h}\left\|v_{k}(\cdot, t+h)-v_{k}(\cdot, t)\right\|_{L^{2}(B)}^{2} \mathrm{~d} t<\theta
$$

which proves (4.9).

Since the sequence $\left(v_{k}\right)_{k \in \mathbb{N}}$ is also bounded in $L^{2}\left(-1,0 ; W^{1,2}\left(B, \mathbb{R}^{N}\right)\right)$ we are able to apply Theorem 3 of [39] with the choice $X=W^{1,2}\left(B, \mathbb{R}^{N}\right), B=L^{2}\left(B, \mathbb{R}^{N}\right), F=\left(v_{k}\right)_{k \in \mathbb{N}}$ to obtain a subsequence $\left(v_{k}\right)_{k \in \mathbb{N}}$ (again labeled by $k$ ) such that

$$
v_{k} \rightarrow v \quad \text { strongly in } L^{2}\left(Q, \mathbb{R}^{N}\right)=L^{2}\left(-1,0 ; L^{2}\left(B, \mathbb{R}^{N}\right)\right) .
$$

To obtain the desired contradiction we denote by $w_{k}: Q \rightarrow \mathbb{R}^{N}$ a solution to the following initial-Dirichlet problem and possessing the properties below; its existence can be deduced from standard existence arguments [27,28].

$$
\begin{aligned}
& \left\{\begin{array}{l}
w_{k} \in C\left([-1,0] ; L^{2}\left(B, \mathbb{R}^{N}\right)\right) \cap L^{2}\left(-1,0 ; W_{0}^{1,2}\left(B, \mathbb{R}^{N}\right)\right), \\
\partial_{t} w_{k} \in L^{2}\left(-1,0 ; W^{-1,2}\left(B, \mathbb{R}^{N}\right)\right), \\
w_{k}(\cdot,-1)=0
\end{array}\right. \\
& \int_{Q}\left(w_{k} \varphi_{t}-A_{k}\left(D w_{k}, D \varphi\right)\right) \mathrm{d} z=\int_{Q}\left(A-A_{k}\right)(D v, D \varphi) \mathrm{d} z \quad \forall \varphi \in C_{0}^{\infty}\left(Q, \mathbb{R}^{N}\right) ; \\
& \left\{\begin{array}{c}
\frac{1}{2}\left\|w_{k}(\cdot, t)\right\|_{L^{2}(B)}^{2}+\int_{B \times(-1, t)} A_{k}\left(D w_{k}, D w_{k}\right) \mathrm{d} z \\
=\int_{B \times(-1, t)}\left(A_{k}-A\right)\left(D v, D w_{k}\right) \mathrm{d} z \quad \text { for a.e. } t \in(-1,0) .
\end{array}\right.
\end{aligned}
$$

Using the ellipticity of the bilinear forms $A_{k}$ we see that the second term of the left-hand side of (4.10) is bounded from below by $\lambda \int_{B \times(-1, t)}\left|D w_{k}\right|^{2} \mathrm{~d} z$. Moreover the right-hand side of (4.10) is estimated easily by the use of Cauchy-Schwarz inequality, the bound $\int_{Q}|D v|^{2} \mathrm{~d} z \leqslant 1$ from (4.5), and Young's inequality

$$
\begin{aligned}
\int_{B \times(-1, t)}\left(A_{k}-A\right)\left(D v, D w_{k}\right) \mathrm{d} z & \leqslant\left|A-A_{k}\right|\left(\int_{Q}|D v|^{2} \mathrm{~d} z\right)^{1 / 2}\left(\int_{B \times(-1, t)}\left|D w_{k}\right|^{2} \mathrm{~d} z\right)^{1 / 2} \\
& \leqslant \frac{2}{\lambda}\left|A-A_{k}\right|^{2}+\frac{\lambda}{2} \int_{B \times(-1, t)}\left|D w_{k}\right|^{2} \mathrm{~d} z .
\end{aligned}
$$

This implies in particular

$$
\frac{1}{2} \int_{B}\left|w_{k}(\cdot, t)\right|^{2} \mathrm{~d} x+\frac{\lambda}{2} \int_{B \times(-1, t)}\left|D w_{k}\right|^{2} \mathrm{~d} z \leqslant \frac{2}{\lambda}\left|A_{k}-A\right|^{2} \quad \text { for a.e. } t \in[-1,0] \text { and } k \in \mathbb{N} .
$$

Taking the supremum over $t \in(-1,0)$ we arrive at

$$
\sup _{t \in(-1,0)} \frac{1}{2} \int_{B}\left|w_{k}(\cdot, t)\right|^{2} \mathrm{~d} x+\frac{\lambda}{2} \int_{Q}\left|D w_{k}\right|^{2} \mathrm{~d} z \rightarrow 0 \quad \text { as } k \rightarrow \infty .
$$


Letting $g_{k}:=v-w_{k} \in L^{2}\left(-1,0, W^{1,2}\left(B, \mathbb{R}^{N}\right)\right)$ we easily see that $g_{k}$ agrees with $v$ on the parabolic boundary $\partial_{p} Q$ of $Q$ and satisfies

$$
\int_{Q} g_{k} \varphi_{t}-A_{k}\left(D g_{k}, D \varphi\right) \mathrm{d} z=0, \quad \forall \varphi \in C_{0}^{\infty}\left(Q, \mathbb{R}^{N}\right) .
$$

From (4.11) and the definition of $g_{k}$ we see that

$$
\int_{Q}\left(\left|g_{k}-v\right|^{2}+\left|D g_{k}-D v\right|^{2}\right) \mathrm{d} z \rightarrow 0 \quad \text { as } k \rightarrow \infty
$$

which implies in particular that

$$
\int_{Q}\left(\left|g_{k}\right|^{2}+\left|D g_{k}\right|^{2}\right) \mathrm{d} z \rightarrow \int_{Q}\left(|v|^{2}+|D v|^{2}\right) \mathrm{d} z \leqslant 1 \quad \text { as } k \rightarrow \infty .
$$

Letting

$$
b_{k}:=\max \left\{1, \int_{Q}\left(\left|g_{k}\right|^{2}+\left|D g_{k}\right|^{2}\right) \mathrm{d} z\right\}, \quad \tilde{g}_{k}:=\frac{g_{k}}{b_{k}}
$$

we see that $b_{k} \rightarrow 1$ and $\int_{Q}\left(\left|\tilde{g}_{k}\right|^{2}+\left|D \tilde{g}_{k}\right|^{2}\right) \mathrm{d} z \leqslant 1$ for any $k \in \mathbb{N}$. Note that $\tilde{g}_{k} \in \mathcal{H}_{k}$. Furthermore,

$$
\left(\int_{Q}\left|\tilde{g}_{k}-v\right|^{2} \mathrm{~d} z\right)^{1 / 2} \leqslant\left(\int_{Q}\left|g_{k}-v\right|^{2} \mathrm{~d} z\right)^{1 / 2}+\left(1-\frac{1}{b_{k}}\right)\left(\int_{Q}\left|g_{k}\right|^{2} \mathrm{~d} z\right)^{1 / 2} \rightarrow 0 \text { as } k \rightarrow \infty,
$$

which yields the desired contradiction to (4.3).

Remark 4.2. From (4.7) we infer

$$
\int_{-1}^{-h}\left\|v_{k}(\cdot, t+h)-v_{k}(\cdot, t)\right\|_{W^{-\ell, 2(B)}} \mathrm{d} t \leqslant c\left(a \sqrt{h}+\frac{1}{k}\right)
$$

which yields, reasoning as above

$$
\int_{-1}^{-h}\left\|v_{k}(\cdot, t+h)-v_{k}(\cdot, t)\right\|_{W^{-\ell, 2(B)}} \mathrm{d} t \rightarrow 0 \quad \text { uniformly in } k \in \mathbb{N} \text { as } h \downarrow 0 .
$$

Therefore we could have had directly applied Theorem 5 of [39] with the choice $X=W^{1,2}\left(B, \mathbb{R}^{N}\right), B=$ $L^{2}\left(B, \mathbb{R}^{N}\right), Y=W^{-\ell, 2}\left(B, \mathbb{R}^{N}\right), F=\left(v_{k}\right)_{k \in \mathbb{N}}, p=2$ to conclude that $\left(v_{k}\right)_{k \in \mathbb{N}}$ is relatively compact in $L^{2}\left(Q, \mathbb{R}^{N}\right)$ $=L^{2}\left(-1,0 ; L^{2}\left(B, \mathbb{R}^{N}\right)\right)$.

Lemma 4.3. There exists a positive function $\delta(n, N, \lambda, \Lambda, \varepsilon) \leqslant 1$ with the following property: Whenever $A$ is a bilinear form on $\mathbb{R}^{n N}$ which is strongly elliptic with ellipticity constant $\lambda>0$ and upper bound $\Lambda, \varepsilon$ is a positive number, and $u \in L^{2}\left(t_{0}-\varrho^{2}, t_{0} ; W^{1,2}\left(B_{\varrho}\left(x_{0}\right), \mathbb{R}^{N}\right)\right)$ with

$$
\varrho^{-2} f_{Q_{\varrho}\left(z_{0}\right)}|u|^{2} \mathrm{~d} z+f_{Q_{\varrho}\left(z_{0}\right)}|D u|^{2} \mathrm{~d} z \leqslant 1
$$


is approximatively A-caloric in the sense that

$$
\left|f_{Q_{\varrho}\left(z_{0}\right)}\left(u \varphi_{t}-A(D u, D \varphi)\right) \mathrm{d} z\right| \leqslant \delta \sup _{Q_{\varrho}\left(z_{0}\right)}|D \varphi| \text { for all } \varphi \in C_{0}^{\infty}\left(Q_{\varrho}\left(z_{0}\right), \mathbb{R}^{N}\right)
$$

then there exists $h \in L^{2}\left(t_{0}-\varrho^{2}, t_{0} ; W^{1,2}\left(B_{\varrho}\left(x_{0}\right), \mathbb{R}^{N}\right)\right)$ A-caloric on $Q_{\varrho}\left(z_{0}\right)$ such that

$$
\varrho^{-2} f_{Q_{\varrho}\left(z_{0}\right)}|h|^{2} \mathrm{~d} z+f_{Q_{\varrho}\left(z_{0}\right)}|D h|^{2} \mathrm{~d} z \leqslant 1 \text { and } \varrho^{-2} f_{Q_{\varrho}\left(z_{0}\right)}|u-h|^{2} \mathrm{~d} z \leqslant \varepsilon .
$$

Proof. For a general parabolic cylinder $Q\left(z_{0}, \varrho\right)=B_{\varrho}\left(x_{0}\right) \times\left(t_{0}-\varrho^{2}, t_{0}\right)$ we can apply Lemma 4.1 (in a suitable averaged version) to the rescaled function $v(x, t):=\varrho^{-1} u\left(x_{0}+\varrho x, t_{0}+\varrho^{2} t\right)$ defined on $Q$ to obtain the existence of an $A$-caloric $H: Q \rightarrow \mathbb{R}^{N}$ satisfying (4.12) on $Q_{\varrho} \equiv Q$. Rescaling via $h(z):=\varrho H\left(\varrho^{-1}\left(x-x_{0}\right), \varrho^{-2}\left(t-t_{0}\right)\right)$ yields the desired result.

\section{The Caccioppoli inequality}

From the definitions given in Section 2 we set

$$
H(s)=K(s)(1+s) .
$$

Now we are ready to derive the following Caccioppoli inequality which slightly differs from the usual ones in that it shows the correct dependence on $H(M)$, a fact that will be needed later.

Lemma 5.1. Let $u \in L^{2}\left(-T, 0 ; W^{1,2}\left(\Omega, \mathbb{R}^{N}\right)\right)$ be a weak solution to (1.1) under the assumptions (2.1)-(2.3). Then, for any $M>0$, any affine function $\ell(z) \equiv \ell(x)$ independent of $t$ and satisfying $\left|\ell\left(z_{0}\right)\right|+|D \ell| \leqslant M$, and any $Q_{\varrho}\left(z_{0}\right) \Subset Q_{T}$ with $\varrho \leqslant 1$ we have

$$
f_{Q_{\varrho} / 2\left(z_{0}\right)}|D u-D \ell|^{2} \mathrm{~d} z \leqslant c_{\operatorname{Cacc}}\left(f_{Q_{\varrho}\left(z_{0}\right)}\left|\frac{u-\ell}{\varrho}\right|^{2} \mathrm{~d} z+\varrho^{2 \beta}\right),
$$

where the constant $c_{\text {Cacc }}$ depends only on $\lambda, L$ and $H(M)$.

Proof. The following calculations will be a bit sloppy. To proceed in a rigorous way, one should use a smoothing procedure in time via a family of non-negative mollifying functions or via Steklov averages. Since this is a standard argument and yields only technical minor changes we shall proceed formally.

In (2.10) we take the test-function $\varphi=\eta^{2} \zeta^{2}(u-\ell)$, where $\eta \in C_{0}^{1}\left(B_{\varrho}\left(x_{0}\right)\right)$ is a cut-off function in space such that $0 \leqslant \eta \leqslant 1, \eta \equiv 1$ in $B_{\varrho / 2}\left(x_{0}\right)$ and $|D \eta| \leqslant 4 \varrho^{-1}$ while $\zeta \in C^{1}(\mathbb{R})$ is a cut-off function in time such that, with $0<\varepsilon<\varrho^{2} / 4$ being arbitrary

$$
\begin{cases}\zeta \equiv 1, & \text { on }\left(t_{0}-\frac{\varrho^{2}}{4}, t_{0}-\varepsilon^{2}\right), \\ \zeta \equiv 0, & \text { on }\left(-\infty, t_{0}-\varrho^{2}\right) \cup\left(t_{0}, \infty\right), \\ 0 \leqslant \zeta \leqslant 1, & \text { on } \mathbb{R}, \\ \zeta_{t} \leqslant 0, & \text { on }\left(t_{0}-\frac{\varrho^{2}}{4}, \infty\right), \\ \left|\zeta_{t}\right| \leqslant \frac{3}{\varrho^{2}}, & \text { on }\left(t_{0}-\varrho^{2}, t_{0}-\frac{\varrho^{2}}{4}\right) .\end{cases}
$$


Inserting $\varphi$ in (2.10) we obtain

$$
\begin{aligned}
& \int_{Q_{\varrho}\left(z_{0}\right)} A(z, u, D u) D(u-\ell) \zeta^{2} \eta^{2} \mathrm{~d} z \\
& \quad=-2 \int_{Q_{\varrho}\left(z_{0}\right)} A(z, u, D u) \zeta^{2} \eta \nabla \eta \otimes(u-\ell) \mathrm{d} z+\int_{Q_{\varrho}\left(z_{0}\right)} u \partial_{t} \varphi \mathrm{d} z .
\end{aligned}
$$

We further have

$$
-\int_{Q_{\varrho}\left(z_{0}\right)} A(z, u, D \ell) D(u-\ell) \zeta^{2} \eta^{2} \mathrm{~d} z=2 \int_{Q_{\varrho}\left(z_{0}\right)} A(z, u, D \ell) \zeta^{2} \eta \nabla \eta \otimes(u-\ell) \mathrm{d} z-\int_{Q_{\varrho}\left(z_{0}\right)} A(z, u, D \ell) D \varphi \mathrm{d} z
$$

and

$$
0=\int_{Q_{\varrho}\left(z_{0}\right)} A\left(z_{0}, \ell\left(z_{0}\right), D \ell\right) D \varphi \mathrm{d} z=0 .
$$

Adding the last and second-last equation to (5.3) and using also that $\ell_{t} \equiv 0$ we deduce

$$
\begin{aligned}
\int_{Q_{\varrho}\left(z_{0}\right)} & (A(z, u, D u)-A(z, u, D \ell)) D(u-\ell) \zeta^{2} \eta^{2} \mathrm{~d} z \\
= & -2 \int_{Q_{\varrho}\left(z_{0}\right)}(A(z, u, D u)-A(z, u, D \ell)) \zeta^{2} \eta \nabla \eta \otimes(u-\ell) \mathrm{d} z \\
& -\int_{Q_{\varrho}\left(z_{0}\right)}(A(z, u, D \ell)-A(z, \ell(z), D \ell)) D \varphi \mathrm{d} z \\
& -\int_{Q_{\varrho}\left(z_{0}\right)}\left(A(z, \ell(z), D \ell)-A\left(z_{0}, \ell\left(z_{0}\right), D \ell\right)\right) D \varphi \mathrm{d} z+\int_{Q_{\varrho}\left(z_{0}\right)}(u-\ell) \partial_{t} \varphi \mathrm{d} z \\
=: & I+I I+I I I+I V .
\end{aligned}
$$

Estimate for $I$ : The Lipschitz bound $|A(z, u, p)-A(z, u, \tilde{p})| \leqslant L|p-\tilde{p}|$ for $p, \tilde{p} \in \mathbb{R}^{n N}$ and an application of Young's inequality yield (for $0<\mu \leqslant 1$ to be chosen later)

$$
\begin{aligned}
|I| & \leqslant 2 L \int_{Q_{\varrho}\left(z_{0}\right)}|D u-D \ell| \zeta^{2} \eta|\nabla \eta||(u-\ell)| \mathrm{d} z \\
& \leqslant \mu \int_{Q_{\varrho}\left(z_{0}\right)}|D u-D \ell|^{2} \zeta^{2} \eta^{2} \mathrm{~d} z+\frac{c(L)}{\mu} \int_{Q_{\varrho}\left(z_{0}\right)} \zeta^{2}|\nabla \eta|^{2}|u-\ell|^{2} \mathrm{~d} z \\
& \leqslant \mu \int_{Q_{\varrho}\left(z_{0}\right)}|D u-D \ell|^{2} \zeta^{2} \eta^{2} \mathrm{~d} z+\frac{c(L)}{\mu} \int_{Q_{\varrho}\left(z_{0}\right)}\left|\frac{u-\ell}{\varrho}\right|^{2} \mathrm{~d} z .
\end{aligned}
$$

Estimate for $I V$ : Using the fact that $\zeta \equiv 0$ on $\left(-\infty, t_{0}-\varrho^{2}\right) \cup\left(t_{0}, \infty\right)$, the integral $I V$ can formally be rewritten in the form (we note that the smoothing procedure mentioned at the beginning of the proof justifies this formal computation) 


$$
\begin{aligned}
I V & =\int_{Q_{\varrho}\left(z_{0}\right)}(u-\ell) \partial_{t} \varphi \mathrm{d} z=\int_{Q_{\varrho}\left(z_{0}\right)}|u-\ell|^{2} \eta^{2} \partial_{t} \zeta^{2} \mathrm{~d} z+\frac{1}{2} \int_{Q_{\varrho}\left(z_{0}\right)} \zeta^{2} \eta^{2} \partial_{t}|u-\ell|^{2} \mathrm{~d} z \\
& =\frac{1}{2} \int_{Q_{\varrho}\left(z_{0}\right)}|u-\ell|^{2} \eta^{2} \partial_{t} \zeta^{2} \mathrm{~d} z=\int_{Q_{\varrho}\left(z_{0}\right)}|u-\ell|^{2} \eta^{2} \zeta \zeta_{t} \mathrm{~d} z .
\end{aligned}
$$

Taking into account that $\zeta \zeta_{t} \leqslant 0$ for $t>t_{0}-\varrho^{2} / 4$ and that $\left|\zeta_{t}\right| \leqslant 3 \varrho^{-2}$ we infer

$$
I V \leqslant 3 \int_{Q_{\varrho}\left(z_{0}\right)}\left|\frac{u-\ell}{\varrho}\right|^{2} \mathrm{~d} z .
$$

Estimate for II: Using the assumption imposed for the modulus of continuity of $(z, u) \mapsto A(z, u, p)$, i.e. (2.6), we see that

$$
|I I| \leqslant H(M) \int_{Q_{\varrho}\left(z_{0}\right)}|u-\ell|^{\beta}|D \varphi| \mathrm{d} z \leqslant I I_{1}+I I_{2}
$$

where

$$
\begin{aligned}
& I_{1}:=H(M) \int_{Q_{\varrho}\left(z_{0}\right)}|u-\ell|^{\beta} \zeta^{2} \eta^{2}|D(u-\ell)| \mathrm{d} z, \\
& I_{2}:=2 H(M) \int_{Q_{\varrho}\left(z_{0}\right)}|u-\ell|^{\beta} \zeta^{2} \eta|\nabla \eta||u-\ell| \mathrm{d} z .
\end{aligned}
$$

To estimate $I I_{1}$ we use Young's inequality twice

$$
\begin{aligned}
I I_{1} & \leqslant \mu \int_{Q_{\varrho}\left(z_{0}\right)} \eta^{2} \zeta^{2}|D(u-\ell)|^{2} \mathrm{~d} z+\frac{1}{\mu} H(M)^{2} \varrho^{2 \beta} \int_{Q_{\varrho}\left(z_{0}\right)}\left|\frac{u-\ell}{\varrho}\right|^{2 \beta} \mathrm{d} z \\
& \leqslant \mu \int_{Q_{\varrho}\left(z_{0}\right)} \eta^{2}|D(u-\ell)|^{2} \mathrm{~d} z+\frac{1}{\mu} H(M)^{2}\left(\left|Q_{\varrho}\left(z_{0}\right)\right| \varrho^{2 \beta /(1-\beta)}+\int_{Q_{\varrho}\left(z_{0}\right)}\left|\frac{u-\ell}{\varrho}\right|^{2} \mathrm{~d} z\right) .
\end{aligned}
$$

Arguing similarly we obtain

$$
I_{2} \leqslant 8 H(M)\left(\left|Q_{\varrho}\left(z_{0}\right)\right| \varrho^{2 \beta /(1-\beta)}+\int_{Q_{\varrho}\left(z_{0}\right)}\left|\frac{u-\ell}{\varrho}\right|^{2} \mathrm{~d} z\right) .
$$

Estimate for III: To estimate III we proceed as follows: Using (2.6) again we see that

$$
I I I \leqslant 2 H(M)^{1+\beta} \varrho^{\beta} \int_{Q_{\varrho}\left(z_{0}\right)}|D \varphi| \mathrm{d} z \leqslant I I I_{1}+I I I_{2}
$$

where

$$
\begin{aligned}
& I I I_{1}:=2 H(M)^{1+\beta} \varrho^{\beta} \int_{Q_{\varrho}\left(z_{0}\right)} \zeta^{2} \eta^{2}|D(u-\ell)| \mathrm{d} z, \\
& I I I_{2}:=4 H(M)^{1+\beta} \varrho^{\beta} \int_{Q_{\varrho}\left(z_{0}\right)} \zeta^{2} \eta|\nabla \eta||u-\ell| \mathrm{d} z .
\end{aligned}
$$


Now, using Young's inequality twice again, we have

$$
I I I_{1}+I I I_{2} \leqslant \mu \int_{Q_{\varrho}\left(z_{0}\right)} \eta^{2} \zeta^{2}|D(u-\ell)|^{2} \mathrm{~d} z+\frac{c}{\mu} H(M)^{2(1+\beta)}\left(\left|Q_{\varrho}\left(z_{0}\right)\right| \varrho^{2 \beta}+\int_{Q_{\varrho}\left(z_{0}\right)}\left|\frac{u-\ell}{\varrho}\right|^{2} \mathrm{~d} z\right) .
$$

Combining (5.5), (5.7)-(5.9), (5.6) with (5.4) and using the fact that $\varrho^{2 \beta /(1-\beta)} \leqslant \varrho^{2 \beta}$ for $0<\varrho \leqslant 1$ we arrive at

$$
\begin{aligned}
& \int_{Q_{\varrho}\left(z_{0}\right)}(A(z, u, D u)-A(z, u, D \ell)) D(u-\ell) \zeta^{2} \eta^{2} \mathrm{~d} z \\
& \quad \leqslant 3 \mu \int_{Q_{\varrho}\left(z_{0}\right)}|D u-D \ell|^{2} \zeta^{2} \eta^{2} \mathrm{~d} z+\frac{c(L, H(M))}{\mu}\left(\int_{Q_{\varrho}\left(z_{0}\right)}\left|\frac{u-\ell}{\varrho}\right|^{2} \mathrm{~d} z+\left|Q_{\varrho}\left(z_{0}\right)\right| \varrho^{2 \beta}\right) .
\end{aligned}
$$

We next estimate the integral on the left-hand side of the previous inequality using the ellipticity condition for the coefficients $A$, which in turns implies strict monotonicity, i.e. $(A(z, u, p)-A(z, u, \tilde{p}))(p-\tilde{p}) \geqslant \lambda|p-\tilde{p}|^{2}$ for any $p, \tilde{p} \in \mathbb{R}^{n N}$. We therefore obtain

$$
(\lambda-3 \mu) \int_{Q_{\varrho}\left(z_{0}\right)}|D u-D \ell|^{2} \eta^{2} \zeta^{2} \mathrm{~d} z \leqslant \frac{c(L, H(M))}{\mu}\left(\int_{Q_{\varrho}\left(z_{0}\right)}\left|\frac{u-\ell}{\varrho}\right|^{2} \mathrm{~d} z+\left|Q_{\varrho}\left(z_{0}\right)\right| \varrho^{2 \beta}\right) .
$$

Choosing $\mu$ small enough, i.e. $\mu=\min (1, \lambda / 6)$, and taking into account that $\zeta \equiv 1$ for $t \in\left[t_{0}-\varrho^{2} / 4, t_{0}-\varepsilon^{2}\right]$, that $\eta \equiv 1$ on $B_{\frac{\varrho}{2}}\left(x_{0}\right)$ we infer that

$$
\int_{t_{0}-\varrho^{2} / 4}^{t_{0}-\varepsilon^{2}} \int_{B_{\varrho / 2}\left(x_{0}\right)}|D u-D \ell|^{2} \mathrm{~d} z \leqslant c(\lambda, L, H(M))\left(\int_{Q_{\varrho}\left(z_{0}\right)}\left|\frac{u-\ell}{\varrho}\right|^{2} \mathrm{~d} z+\left|Q_{\varrho}\left(z_{0}\right)\right| \varrho^{2 \beta}\right) .
$$

Now, the desired Caccioppoli inequality follows by taking the limit $\varepsilon \rightarrow 0$.

Remark 5.2. Remark 2.1 and a careful inspection of the previous proof, reveal that in the case of systems of the type (1.4) the term II drops out while the estimate for III simplifies. Therefore the only condition we have to take on $\ell(z)$ is that $|D \ell| \leqslant M$.

\section{Linearization}

The next inequality will later allow us to apply the $A$-caloric approximation lemma.

Lemma 6.1. Let $u \in L^{2}\left(-T, 0 ; W^{1,2}\left(\Omega, \mathbb{R}^{N}\right)\right)$ be a weak solution to (1.1) under the assumptions (2.1)-(2.3) and (2.9). Then for any $M>0$ we have

$$
\begin{aligned}
& \left|\underset{Q_{\varrho}\left(z_{0}\right)}{f}\left((u-\ell) \varphi_{t}-\frac{\partial A}{\partial p}\left(z_{0}, \ell\left(z_{0}\right), D \ell\right)(D u-D \ell) D \varphi\right) \mathrm{d} z\right| \\
& \quad \leqslant c_{E u}\left(\omega\left(M+1, \Phi_{2}\right) \sqrt{\Phi_{2}}+\Phi_{2}+\Psi_{2}+\varrho^{\beta}\right) \sup _{Q_{\varrho}\left(z_{0}\right)}|D \varphi|,
\end{aligned}
$$

for any $Q_{\varrho}\left(z_{0}\right) \Subset Q_{T}$ and $\varphi \in C_{0}^{\infty}\left(Q_{\varrho}\left(z_{0}\right), \mathbb{R}^{N}\right)$ with $\varrho \leqslant 1$ and any affine function $\ell(z)=\ell(x)$ independent of time, satisfying $\left|\ell\left(z_{0}\right)\right|+|D \ell| \leqslant M$. Here $c_{E u} \equiv c(H(M), L)$ (the function $H$ is defined in (5.1)). We write 


$$
\begin{aligned}
& \Phi_{2} \equiv \Phi_{2}\left(z_{0}, \varrho, D \ell\right):=\int_{Q_{\varrho}\left(z_{0}\right)}|D u-D \ell|^{2} \mathrm{~d} z \\
& \Psi_{2} \equiv \Psi_{2}\left(z_{0}, \varrho, \ell\right):=f_{Q_{\varrho}\left(z_{0}\right)}\left|\frac{u-\ell}{\varrho}\right|^{2} \mathrm{~d} z .
\end{aligned}
$$

Proof. Without loss of generality we can assume that $\sup _{Q_{\varrho}\left(z_{0}\right)}|D \varphi| \leqslant 1$. Using (2.10), the fact that

$$
f_{Q_{\varrho}\left(z_{0}\right)} A\left(z_{0}, \ell\left(z_{0}\right), D \ell\right) D \varphi \mathrm{d} z=0,
$$

and $f_{Q_{\varrho}\left(z_{0}\right)} \ell \varphi_{t} \mathrm{~d} z=0$ we deduce

$$
f_{Q_{\varrho}\left(z_{0}\right)}\left((u-\ell) \varphi_{t}-\frac{\partial A}{\partial p}\left(z_{0}, \ell\left(z_{0}\right), D \ell\right)(D u-D \ell) D \varphi\right) \mathrm{d} z=I+I I+I I I,
$$

where we have abbreviated

$$
\begin{aligned}
I:= & \int_{Q_{\varrho}\left(z_{0}\right)}\left(A\left(z_{0}, \ell\left(z_{0}\right), D u\right)-\frac{\partial A}{\partial p}\left(z_{0}, \ell\left(z_{0}\right), D \ell\right)(D u-D \ell) D \varphi\right) \mathrm{d} \tau \mathrm{d} z \\
I I & :=f_{Q_{\varrho}\left(z_{0}\right)}(A(z, u, D u)-A(z, \ell, D u)) D \varphi \mathrm{d} z, \\
I I I & :=f_{Q_{\varrho}\left(z_{0}\right)}\left(A(z, \ell, D u)-A\left(z_{0}, \ell\left(z_{0}\right), D u\right)\right) D \varphi \mathrm{d} z .
\end{aligned}
$$

In turn we split the first integral as follows

$$
I=\frac{1}{\left|Q_{\varrho}\left(z_{0}\right)\right|} \int_{S_{1}}(\cdots) \mathrm{d} z+\frac{1}{\left|Q_{\varrho}\left(z_{0}\right)\right|} \int_{S_{2}}(\cdots) \mathrm{d} z=: I V+V
$$

and

$$
S_{1}:=Q_{\varrho}\left(z_{0}\right) \cap\{z:|D u-D \ell| \leqslant 1\}, \quad S_{2}:=Q_{\varrho}\left(z_{0}\right) \cap\{z:|D u-D \ell|>1\} .
$$

We proceed estimating the two resulting pieces. As for $I V$ we write

$$
I V=\frac{1}{\left|Q_{\varrho}\left(z_{0}\right)\right|} \int_{S_{1}}\left[\int_{0}^{1}\left[\frac{\partial A}{\partial p}\left(z_{0}, \ell\left(z_{0}\right), D \ell+\tau(D u-D \ell)\right)-\frac{\partial A}{\partial p}\left(z_{0}, \ell\left(z_{0}\right), D \ell\right)\right](D u-D \ell) D \varphi\right] \mathrm{d} \tau \mathrm{d} z .
$$

We estimate $I V$ using the modulus of continuity $\omega(\cdot, \cdot)$ for $(z, u, p) \mapsto \frac{\partial A}{\partial p}(z, u, p)$ from (2.7), the fact that $s \mapsto$ $\omega^{2}(t, s)$ is concave, and Jensen's inequality (note that, by assumption, on $S_{1}$ we have $\left|\ell\left(z_{0}\right)\right|+|D \ell|+|D u-D \ell| \leqslant$ $M+1)$ :

$$
|I V| \leqslant \int_{Q_{\varrho}\left(z_{0}\right)} \omega\left(M+1,|D u-D \ell|^{2}\right)|D u-D \ell| \mathrm{d} z
$$




$$
\begin{aligned}
& \leqslant\left(f_{Q_{\varrho}\left(z_{0}\right)} \omega^{2}\left(M+1,|D u-D \ell|^{2}\right) \mathrm{d} z\right)^{1 / 2}\left(f_{Q_{\varrho}\left(z_{0}\right)}|D u-D \ell|^{2} \mathrm{~d} z\right)^{1 / 2} \\
& \leqslant \omega\left(M+1, f_{Q_{\varrho}\left(z_{0}\right)}|D u-D \ell|^{2} \mathrm{~d} z\right)\left(f_{Q_{\varrho}\left(z_{0}\right)}|D u-D \ell|^{2} \mathrm{~d} z\right)^{1 / 2} .
\end{aligned}
$$

To estimate $V$ we preliminarily observe that, using Hölder's inequality

$$
\left|S_{2}\right| \leqslant \int_{S_{2}}|D u-D \ell| \mathrm{d} z \leqslant \sqrt{\left|S_{2}\right|} \sqrt{\left|Q_{\varrho}\left(z_{0}\right)\right|}\left(f_{Q_{\varrho}\left(z_{0}\right)}|D u-D \ell|^{2} \mathrm{~d} z\right)^{1 / 2},
$$

and therefore

$$
\frac{\sqrt{\left|S_{2}\right|}}{\sqrt{\left|Q_{\varrho}\left(z_{0}\right)\right|}} \leqslant\left(f_{Q_{\varrho}\left(z_{0}\right)}|D u-D \ell|^{2} \mathrm{~d} z\right)^{1 / 2} .
$$

Using (2.1), (2.2) and the previous inequality we then conclude the estimate of $V$ as follows

$$
\begin{aligned}
|V| & \leqslant \frac{2 L}{\left|Q_{\varrho}\left(z_{0}\right)\right|} \int_{S_{2}} 1+|D \ell|+|D u-D \ell| \mathrm{d} z \leqslant \frac{2 L}{\left|Q_{\varrho}\left(z_{0}\right)\right|} \int_{S_{2}} 1+M+|D u-D \ell| \mathrm{d} z \\
& \leqslant 2 L(M+1) \frac{\left|S_{2}\right|}{\left|Q_{\varrho}\left(z_{0}\right)\right|}+2 L \frac{\sqrt{\left|S_{2}\right|}}{\sqrt{\left|Q_{\varrho}\left(z_{0}\right)\right|}}\left(f_{Q_{\varrho}\left(z_{0}\right)}|D u-D \ell|^{2} \mathrm{~d} z\right)^{1 / 2} \\
& \leqslant 4 L(M+1) f_{Q_{\varrho}\left(z_{0}\right)}|D u-D \ell|^{2} \mathrm{~d} z .
\end{aligned}
$$

Combining the estimates found for $I V$ and $V$ we have

$$
|I| \leqslant \omega\left(M+1, \Phi_{2}\right) \sqrt{\Phi_{2}}+4 L(M+1) \Phi_{2} .
$$

For the remaining pieces, using the modulus of continuity $(z, u) \mapsto A(z, u, p)$ from (2.6), we deduce

$$
\begin{aligned}
|I I| & \leqslant K\left(\left|\ell\left(z_{0}\right)\right|+|D \ell|\right) f_{Q_{\varrho}\left(z_{0}\right)}|u-\ell|^{\beta}(1+|D \ell|+|D u-D \ell|) \mathrm{d} z \\
& \leqslant H(M) \varrho^{\beta} f_{Q_{\varrho}\left(z_{0}\right)}\left|\frac{u-\ell}{\varrho}\right|^{\beta} \mathrm{d} z+H(M) \varrho^{\beta} f_{Q_{\varrho}\left(z_{0}\right)}\left|\frac{u-\ell}{\varrho}\right|^{\beta}|D u-D \ell| \mathrm{d} z \\
& \leqslant 2 H(M)\left[f_{Q_{\varrho}\left(z_{0}\right)}|D u-D \ell|^{2} \mathrm{~d} z+f_{Q_{\varrho}\left(z_{0}\right)}\left|\frac{u-\ell}{\varrho}\right|^{2} \mathrm{~d} z+\varrho^{\beta}\right] .
\end{aligned}
$$

Here we have used that $1 \leqslant K(M) \leqslant H(M)$ and the assumption that $\varrho \leqslant 1$. Using again (2.6) and Young's inequality we estimate

$$
|I I I| \leqslant 2^{\beta} K\left(\left|\ell\left(z_{0}\right)\right|\right)(1+|D \ell|)^{\beta} \varrho^{\beta} f_{Q_{\varrho}\left(z_{0}\right)}(1+|D \ell|+|D u-D \ell|) \mathrm{d} z
$$




$$
\begin{aligned}
& \leqslant 2 H(M)^{2} \varrho^{\beta}+2 H(M)\left(\varrho^{2 \beta}+f_{Q_{\varrho}\left(z_{0}\right)}|D u-D \ell|^{2} \mathrm{~d} z\right) \\
& \leqslant 3 H(M)^{2}\left(\varrho^{\beta}+f_{Q_{\varrho}\left(z_{0}\right)}|D u-D \ell|^{2} \mathrm{~d} z\right) .
\end{aligned}
$$

Combining the estimates just found for $I, I I$ and $I I I$, we obtain

$$
\begin{aligned}
& \left|\underset{Q_{\varrho}\left(z_{0}\right)}{f}\left((u-\ell) \varphi_{t}-\frac{\partial A}{\partial p}\left(z_{0}, \ell\left(z_{0}\right), D \ell\right)(D u-D \ell) D \varphi\right) \mathrm{d} z\right| \\
& \quad \leqslant c(H(M), L)\left[\omega\left(M+1, \Phi_{2}\right) \sqrt{\Phi_{2}}+\Phi_{2}+\Psi_{2}+\varrho^{\beta}\right] .
\end{aligned}
$$

A simple scaling argument yields the result for general $\varphi$.

Remark 6.2. The same observations on the affine function $\ell(z)$ in Remark 5.2 apply to the previous proof; therefore, in the case of systems (1.4), once again we may assume only that $|D \ell| \leqslant M$.

The next lemma is a standard estimate for weak solutions to linear parabolic systems with constant coefficients [4], Lemma 5.1.

Lemma 6.3. Let $h \in L^{2}\left(t_{0}-\varrho^{2}, t_{0} ; W^{1,2}\left(B_{\varrho}\left(x_{0}\right), \mathbb{R}^{N}\right)\right)$ be a weak solution in $Q_{\varrho}\left(z_{0}\right)=B_{\varrho}\left(x_{0}\right) \times\left(t_{0}-\varrho^{2}, t_{0}\right)$ of the following linear parabolic system with constant coefficients:

$$
f_{Q_{\varrho}\left(z_{0}\right)}\left(h \varphi_{t}-A(D h, D \varphi)\right) \mathrm{d} z=0, \quad \forall \varphi \in C_{0}^{\infty}\left(Q_{\varrho}\left(z_{0}\right), \mathbb{R}^{N}\right),
$$

where the coefficients A satisfy

$$
A(p, p) \geqslant \lambda|p|^{2}, \quad A(p, \tilde{p}) \leqslant L|p \| \tilde{p}|,
$$

for any $p, \tilde{p} \in \mathbb{R}^{n N}$. Then $h$ is smooth in $Q_{\varrho}\left(z_{0}\right)$ and there exists a constant $c_{p a}=c_{p a}(n, N, L / \lambda) \geqslant 1$, such that

$$
\Psi\left(z_{0}, \theta \varrho\right) \leqslant c_{P a} \theta^{2} \Psi\left(z_{0}, \varrho\right) \quad \forall 0<\theta<1 .
$$

Here we write for $0<\sigma \leqslant \varrho$

$$
\Psi\left(z_{0}, \sigma\right)=\frac{1}{\sigma^{2}} f_{Q_{\sigma}\left(z_{0}\right)}\left|h-(h)_{z_{0}, \sigma}-(D h)_{z_{0}, \sigma}\left(x-x_{0}\right)\right|^{2} \mathrm{~d} z .
$$

\section{Regular points}

In this section we consider a weak solution $u$ of the nonlinear parabolic system (1.1) on a fixed sub-cylinder $Q_{\varrho}\left(z_{0}\right) \Subset Q_{T}$, under the assumptions described in Section 2. In the following we shall always consider $\varrho \leqslant 1$.

Let $M>1$ be given. We first want to apply Lemma 6.1 on $Q_{\varrho / 2}\left(z_{0}\right)$ to

$$
v:=u-\ell,
$$

where $\ell(z)=\ell(x)$ is an affine function independent of $t$ satisfying $\left|\ell\left(z_{0}\right)\right|+|D \ell| \leqslant M$. We observe that $\Psi_{2}$ has the following property:

$$
\Psi_{2}\left(z_{0}, \varrho / 2, \ell\right)=f_{Q_{\varrho / 2}\left(z_{0}\right)}\left|\frac{u-\ell}{\varrho / 2}\right|^{2} \mathrm{~d} z \leqslant 2^{n+4} f_{Q_{\varrho}\left(z_{0}\right)}\left|\frac{u-\ell}{\varrho}\right|^{2} \mathrm{~d} z=2^{n+4} \Psi_{2}\left(z_{0}, \varrho, \ell\right) .
$$


From Caccioppoli's inequality (5.2), we infer

$$
\Phi_{2}\left(z_{0}, \varrho / 2, D \ell\right) \leqslant c_{\operatorname{Cacc}}\left(f_{Q_{\varrho}\left(z_{0}\right)}\left|\frac{u-\ell}{\varrho}\right|^{2} \mathrm{~d} z+\varrho^{2 \beta}\right)=c_{\text {Cacc }}\left(\Psi_{2}\left(z_{0}, \varrho, \ell\right)+\varrho^{2 \beta}\right)=c_{\text {Cacc }} \widetilde{\Psi}_{2}\left(z_{0}, \varrho, \ell\right),
$$

where we have abbreviated

$$
\widetilde{\Psi}_{2} \equiv \widetilde{\Psi}_{2}\left(z_{0}, \varrho, \ell\right):=\Psi_{2}\left(z_{0}, \varrho, \ell\right)+\varrho^{2 \beta} .
$$

From Lemma 6.1 we therefore get for any $\varphi \in C_{0}^{\infty}\left(Q_{\varrho / 2}\left(z_{0}\right), \mathbb{R}^{N}\right)$ (note also that $\omega(M+1, c s) \leqslant c \omega(M+1, s)$ for $c \geqslant 1$, since $s \mapsto \omega(M+1, s)$ is concave)

$$
\begin{aligned}
& \left|f_{Q_{\varrho / 2}\left(z_{0}\right)}\left(v \varphi_{t}-\frac{\partial A}{\partial p}\left(z_{0}, \ell\left(z_{0}\right), D \ell\right) D v D \varphi\right) \mathrm{d} z\right| \\
& \quad \leqslant c_{1}\left[\omega\left(M+1, \widetilde{\Psi}_{2}\left(z_{0}, \varrho, \ell\right)\right) \sqrt{\widetilde{\Psi}_{2}\left(z_{0}, \varrho, \ell\right)}+\widetilde{\Psi}_{2}\left(z_{0}, \varrho, \ell\right)+\varrho^{\beta}\right] \sup _{Q_{\ell / 2}\left(z_{0}\right)}|D \varphi|,
\end{aligned}
$$

where $c_{1}=c_{1}(\lambda, L, \beta, H(M))$.

For given $\varepsilon>0$ (to be specified later) we let $\delta=\delta(n, N, \lambda, L, \varepsilon) \in(0,1]$ be the constant from Lemma 4.3. We define

$$
\gamma:=4 c_{1} \sqrt{\Psi_{2}\left(z_{0}, \varrho, \ell\right)+\delta^{-2} \varrho^{2 \beta}} \text { and } \quad w:=\gamma^{-1} v=\gamma^{-1}(u-\ell) .
$$

Then, from (7.3) we deduce that for all $\varphi \in C_{0}^{\infty}\left(Q_{\varrho / 2}\left(z_{0}\right), \mathbb{R}^{N}\right)$ there holds

$$
\begin{aligned}
& f_{Q_{\varrho / 2}\left(z_{0}\right)}\left(w \varphi_{t}-\frac{\partial A}{\partial p}\left(z_{0}, \ell\left(z_{0}\right), D \ell\right) D w D \varphi\right) \mathrm{d} z \\
& \quad \leqslant \frac{1}{4}\left[\omega\left(M+1, \widetilde{\Psi}_{2}\left(z_{0}, \varrho, \ell\right)\right)+\sqrt{\widetilde{\Psi}_{2}\left(z_{0}, \varrho, \ell\right)}+\delta\right] \sup _{Q_{\ell / 2}\left(z_{0}\right)}|D \varphi| \\
& \quad \leqslant\left[\omega^{2}\left(M+1, \widetilde{\Psi}_{2}\left(z_{0}, \varrho, \ell\right)\right)+\widetilde{\Psi}_{2}\left(z_{0}, \varrho, \ell\right)+\frac{1}{2} \delta^{2}\right]^{1 / 2} \sup _{Q_{\varrho / 2}\left(z_{0}\right)}|D \varphi| .
\end{aligned}
$$

Moreover, we estimate using Caccioppoli's inequality (7.2) and (7.1)

$$
\left(\frac{\varrho}{2}\right)^{-2} f_{Q_{\varrho / 2}\left(z_{0}\right)}|w|^{2} \mathrm{~d} z+f_{Q_{\varrho / 2}\left(z_{0}\right)}|D w|^{2} \mathrm{~d} z \leqslant \frac{2^{n+4}+c_{\mathrm{Cacc}}}{16 c_{1}^{2}} \leqslant 1,
$$

provided we have chosen $c_{1} \gg 1$ large enough. We further set

$$
\mathcal{A}(p, \tilde{p}):=\frac{\partial A}{\partial p}\left(z_{0}, \ell\left(z_{0}\right), D \ell\right)(p, \tilde{p}) \quad \forall p, \tilde{p} \in \mathbb{R}^{n N} .
$$

From (2.2) and (2.3) we see that the bilinear form $\mathcal{A}$ satisfies the following conditions:

$$
\lambda|\tilde{p}|^{2} \leqslant A(\tilde{p}, \tilde{p}), \quad A(p, \tilde{p}) \leqslant L|p \| \tilde{p}| \quad \forall p, \tilde{p} \in \mathbb{R}^{n N},
$$

i.e. the bilinear form $\mathcal{A}$ fulfills the assumptions of Lemma 4.3. Therefore (7.4) and (7.5) allow us to apply Lemma 4.3 to $w, \mathcal{A}$ on $Q_{\varrho / 2}\left(z_{0}\right)$. Assuming the smallness condition

$$
\omega^{2}\left(M+1, \widetilde{\Psi}_{2}\left(z_{0}, \varrho, \ell\right)\right)+\widetilde{\Psi}_{2}\left(z_{0}, \varrho, \ell\right) \leqslant \frac{1}{2} \delta^{2}
$$


the application of Lemma 4.3 yields the existence of $h \in L^{2}\left(t_{0}-\varrho^{2} / 4, t_{0} ; W^{1,2}\left(B_{\varrho / 2}\left(x_{0}\right), \mathbb{R}^{N}\right)\right.$ solving the $\mathcal{A}$-heat equation on $Q_{\varrho / 2}\left(z_{0}\right)$ and satisfying

and

$$
\left(\frac{\varrho}{2}\right)^{-2} f_{Q_{\varrho / 2}\left(z_{0}\right)}|h|^{2} \mathrm{~d} z+f_{Q_{\varrho / 2}\left(z_{0}\right)}|D h|^{2} \mathrm{~d} z \leqslant 1
$$

$$
\left(\frac{\varrho}{2}\right)^{-2} f_{Q_{\varrho} / 2\left(z_{0}\right)}|w-h|^{2} \mathrm{~d} z \leqslant \varepsilon
$$

From Lemma 6.3 we recall that $h$ satisfies for any $0<\theta \leqslant 1$ the a priori estimate (note that $c_{p a}=c_{p a}(n, N, \lambda, L)$ $\geqslant 1)$

$$
\begin{aligned}
& \left(\frac{\theta \varrho}{2}\right)^{-2} f_{Q_{\theta \varrho / 2}\left(z_{0}\right)}\left|h-(h)_{z_{0}, \theta \varrho / 2}-(D h)_{z_{0}, \theta \varrho / 2}\left(x-x_{0}\right)\right|^{2} \mathrm{~d} z \\
& \leqslant c_{p a} \theta^{2}\left(\frac{\varrho}{2}\right)^{-2} f_{Q_{\varrho / 2}\left(z_{0}\right)}\left|h-(h)_{z_{0}, \varrho / 2}-(D h)_{z_{0}, \varrho / 2}\left(x-x_{0}\right)\right|^{2} \mathrm{~d} z \\
& \leqslant 3 c_{p a} \theta^{2}\left[\left(\frac{\varrho}{2}\right)^{-2}\left(f_{Q_{\varrho / 2}\left(z_{0}\right)}|h|^{2} \mathrm{~d} z+\left|(h)_{z_{0}, \varrho / 2}\right|^{2}\right)+\left|(D h)_{z_{0}, \varrho / 2}\right|^{2}\right] \\
& \leqslant 6 c_{p a} \theta^{2}\left[\left(\frac{\varrho}{2}\right)^{-2} f_{Q_{\varrho / 2}\left(z_{0}\right)}|h|^{2} \mathrm{~d} z+f_{Q_{\varrho / 2}\left(z_{0}\right)}|D h|^{2} \mathrm{~d} z\right] \leqslant 6 c_{p a} \theta^{2} .
\end{aligned}
$$

Here we have used that $\left|(h)_{z_{0}, \varrho / 2}\right|^{2} \leqslant f_{Q_{\varrho / 2}\left(z_{0}\right)}|h|^{2} \mathrm{~d} z$ that $\left|(D h)_{z_{0}, \varrho / 2}\right|^{2} \leqslant f_{Q_{\varrho / 2}\left(z_{0}\right)}|D h|^{2} \mathrm{~d} z$ and (7.6). Combining previous estimate with (7.7) we deduce

$$
\begin{aligned}
& \left(\frac{\theta \varrho}{2}\right)^{-2} \underset{Q_{\theta \varrho / 2}\left(z_{0}\right)}{f}\left|w-(h)_{z_{0}, \theta \varrho / 2}-(D h)_{z_{0}, \theta \varrho / 2}\left(x-x_{0}\right)\right|^{2} \mathrm{~d} z \\
& \quad \leqslant 2\left(\frac{\theta \varrho}{2}\right)^{-2}\left[f_{Q_{\theta \varrho / 2}\left(z_{0}\right)}|w-h|^{2} \mathrm{~d} z+f_{Q_{\theta \varrho / 2}\left(z_{0}\right)}\left|h-(h)_{z_{0}, \theta \varrho / 2}-(D h)_{z_{0}, \theta \varrho / 2}\left(x-x_{0}\right)\right|^{2} \mathrm{~d} z\right] \\
& \quad \leqslant 2\left[\theta^{-n-4}\left(\frac{\varrho}{2}\right)^{-2} f_{Q_{\varrho / 2}\left(z_{0}\right)}|w-h|^{2} \mathrm{~d} z+6 c_{p a} \theta^{2}\right] \leqslant 12 c_{p a}\left(\theta^{-n-4} \varepsilon+\theta^{2}\right) .
\end{aligned}
$$

Rescaling back to $v$ via $w=\gamma^{-1} v=\gamma^{-1}(u-\ell)$ we arrive at

$$
\left(\frac{\theta \varrho}{2}\right)^{-2} f_{Q_{\theta \varrho /}\left(z_{0}\right)}\left|u-\ell-\gamma\left((h)_{z_{0}, \theta \varrho / 2}+(D h)_{z_{0}, \theta \varrho / 2}\left(x-x_{0}\right)\right)\right|^{2} \mathrm{~d} z \leqslant 12 c_{p a}\left(\theta^{-n-4} \varepsilon+\theta^{2}\right) \gamma^{2}
$$

Next we use the minimizing property of $\ell_{z_{0}, \theta \varrho / 2}$ and recall the definition of $\gamma$. Thereby we find

$$
\left(\frac{\theta \varrho}{2}\right)^{-2} f_{Q_{\theta \varrho / 2}\left(z_{0}\right)}\left|u-\ell_{z_{0}, \theta \varrho / 2}\right|^{2} \mathrm{~d} z \leqslant c_{2}\left(\theta^{-n-4} \varepsilon+\theta^{2}\right)\left(\Psi\left(z_{0}, \varrho, \ell\right)+\delta^{-2} \varrho^{2 \beta}\right),
$$

where $c_{2}=192 c_{p a} c_{1}^{2} \geqslant 1$ depends only on $n, N, \lambda, L, \beta, H(M)$. 
Now, we choose $\varepsilon=\theta^{n+6}$. Then (7.8) yields

$$
\Psi\left(z_{0}, \theta \varrho / 2, \ell_{z_{0}, \theta \varrho / 2}\right) \leqslant 2 c_{2} \theta^{2}\left(\Psi\left(z_{0}, \varrho, \ell\right)+\delta^{-2} \varrho^{2 \beta}\right) .
$$

Given $\beta<\alpha<1$ we choose $0<\theta<1$ such that

$$
2^{1+2 \alpha} c_{2} \theta^{2} \leqslant \theta^{2 \alpha},
$$

i.e. $\theta=\theta(n, N, \lambda, L, \alpha, \beta, H(M))$. This also fixes the constants $\varepsilon=\varepsilon(n, N, \lambda, L, \alpha, \beta, H(M))$ and $\delta=\delta(n, N, \lambda$, $L, \alpha, \beta, H(M)) \in(0,1]$. Thus we have shown

Lemma 7.1. Given $M>0$ and $\beta<\alpha<1$ there exist $\vartheta \in\left(0, \frac{1}{2}\right)$ and $\delta \in(0,1]$ depending only on $n, N, \lambda, L, \alpha, \beta$ and $H(M)$ such that if

$$
\omega^{2}\left(M+1, \widetilde{\Psi}_{2}\left(z_{0}, \varrho, \ell_{z_{0}, \varrho}\right)\right)+\widetilde{\Psi}_{2}\left(z_{0}, \varrho, \ell_{z_{0}, \varrho}\right) \leqslant \frac{1}{2} \delta^{2}
$$

on $Q_{\varrho}\left(z_{0}\right) \Subset Q_{T}$ for some $0<\varrho \leqslant 1$ and such if

$$
\left|\ell_{z_{0}, \varrho}\left(z_{0}\right)\right|+\left|D \ell_{z_{0}, \varrho}\right| \leqslant M
$$

then

$$
\widetilde{\Psi}_{2}\left(z_{0}, \vartheta \varrho, \ell_{z_{0}, \vartheta \varrho}\right) \leqslant \vartheta^{2 \alpha} \widetilde{\Psi}_{2}\left(z_{0}, \varrho, \ell_{z_{0}, \varrho}\right)+c_{3} \varrho^{2 \beta},
$$

where $c_{3}:=1+\delta^{-2}$.

Remark 7.2. Keeping into account the content of Remarks 5.2 and 6.2 we have that in the case of systems of the type (1.4) the condition in (7.9) can be relaxed to $\left|D \ell_{z_{0}, \varrho}\right| \leqslant M$.

We now want to iterate Lemma 7.1; in the following, for fixed $z_{0}$ we shall denote $\ell_{z_{0}, \varrho} \equiv \ell_{\varrho}$. For given $M>1$ (and $\underline{\beta}<\alpha<1$ ) we determine $\delta=\delta(2 M), \vartheta=\vartheta(2 M)$ and $c_{3}=c_{3}(2 M)$ according to Lemma 7.1. Then we can find $\widetilde{\Psi}_{0}(M)>0$ sufficiently small, such that

$$
\omega^{2}\left(2 M, 2 \widetilde{\Psi}_{0}(M)\right)+2 \widetilde{\Psi}_{0}(M) \leqslant \frac{1}{2} \delta^{2}
$$

and

$$
\widetilde{\Psi}_{0}(M) \leqslant \frac{1}{4(n+2)^{2}} M^{2} \vartheta^{n+4}\left(1-\vartheta^{\alpha}\right)^{2} .
$$

Given this we can also find $\varrho_{0}(M) \in(0,1]$ so small that, writing

$$
c_{4}(M):=\frac{c_{3}(2 M)}{\vartheta^{2 \beta}-\vartheta^{2 \alpha}},
$$

we have

$$
c_{4}(M) \varrho_{0}(M)^{2 \beta} \leqslant \min \left\{\delta^{2}, \widetilde{\Psi}_{0}(M), \frac{1}{4(n+2)^{2}} M^{2} \vartheta^{n+4}\left(1-\vartheta^{\beta}\right)^{2}\right\} .
$$

Now, suppose that the conditions

(i) $\left|\ell_{\varrho}\left(z_{0}\right)\right|+\left|D \ell_{\varrho}\right| \leqslant M$,

(ii) $\varrho \leqslant \varrho_{0}(M)$,

(iii) $\widetilde{\Psi}_{2}(\varrho) \leqslant \widetilde{\Psi}_{0}(M)$ 
are satisfied on $Q_{\varrho}\left(z_{0}\right) \Subset Q_{T}$. Then, for $j=1,2,3, \ldots$ we shall show

(I) $)_{j} \widetilde{\Psi}_{2}\left(\vartheta^{j} \varrho\right) \leqslant \vartheta^{2 \alpha j} \widetilde{\Psi}_{2}(\varrho)+c_{4}(M)\left(\vartheta^{j} \varrho\right)^{2 \beta}$,

(II) ${ }_{j}\left|\ell_{\vartheta^{j} \varrho}\left(z_{0}\right)\right|+\left|D \ell_{\vartheta^{j} \varrho}\right| \leqslant 2 M$.

Note first that (I) ${ }_{j}$ combined with (ii), (iii) and (7.12) yields

$\left(\mathbf{I}^{\prime}\right)_{j} \quad \widetilde{\Psi}_{2}\left(\vartheta^{j} \varrho\right) \leqslant 2 \widetilde{\Psi}_{0}(M)$.

We now proceed by induction. We first consider the case $j=1$. From (iii), (7.10) and the monotonicity of $\omega$ we infer

$$
\omega^{2}\left(M, \widetilde{\Psi}_{2}(\varrho)\right)+\widetilde{\Psi}_{2}(\varrho) \leqslant \omega^{2}\left(2 M, \widetilde{\Psi}_{0}(M)\right)+2 \widetilde{\Psi}_{0}(M) \leqslant \frac{1}{2} \delta^{2} .
$$

Moreover, we have $\varrho \leqslant \varrho_{0}(M) \leqslant 1$ and $\left|\ell_{\varrho}\left(z_{0}\right)\right|+\left|D \ell_{\varrho}\right| \leqslant M$. Therefore we can apply Lemma 7.1 to conclude that $(\mathbf{I})_{1}$ holds. Furthermore, using Lemma 3.1, (iii) and (7.11) we deduce

$$
\begin{aligned}
\left|\ell_{\vartheta \varrho}\left(z_{0}\right)\right|+\left|D \ell_{\vartheta \varrho}\right| & \leqslant\left|\ell_{\varrho}\left(z_{0}\right)\right|+\left|D \ell_{\varrho}\right|+\left|\ell_{\vartheta \varrho}\left(z_{0}\right)-\ell_{\varrho}\left(z_{0}\right)\right|+\left|D \ell_{\vartheta \varrho}-D \ell_{\varrho}\right| \\
& \leqslant M+\left|f_{Q_{\vartheta}\left(z_{0}\right)}\left(u-(u)_{\varrho}\right) \mathrm{d} z\right|+\left[\frac{n(n+2)}{(\vartheta \varrho)^{2}} f_{Q_{\vartheta \varrho}\left(z_{0}\right)}\left|u-\ell_{\varrho}\right|^{2} \mathrm{~d} z\right]^{1 / 2} \\
& \leqslant M+\left[f_{Q_{\vartheta}\left(z_{0}\right)}\left|u-\ell_{\varrho}\right|^{2} \mathrm{~d} z\right]^{1 / 2}+\left[\frac{n(n+2)}{(\vartheta \varrho)^{2}} f_{Q_{\vartheta \varrho}\left(z_{0}\right)}\left|u-\ell_{\varrho}\right|^{2} \mathrm{~d} z\right]^{1 / 2} \\
& \leqslant M+\left[\frac{\vartheta^{2} \varrho^{2}}{\vartheta^{n+4}} \widetilde{\Psi}_{2}(\varrho)\right]^{1 / 2}+\left[\frac{n(n+2)}{\vartheta^{n+4}} \widetilde{\Psi}_{2}(\varrho)\right]^{1 / 2} \leqslant M+\frac{1+\sqrt{n(n+2)}}{\sqrt{\vartheta^{n+4}}} \widetilde{\Psi}_{0}(M)^{1 / 2} \\
& \leqslant M+\frac{n+2}{\sqrt{\vartheta^{n+4}}} \widetilde{\Psi}_{0}(M)^{1 / 2} \leqslant 2 M,
\end{aligned}
$$

i.e. $(\mathbf{I I})_{1}$ holds. We now assume $(\mathbf{I})_{m}$ and $(\mathbf{I I})_{m}$ (and therefore $\left.\left(\mathbf{I}^{\prime}\right)_{m}\right)$ for $m=1, \ldots, j-1$. Then, $\left(\mathbf{I}^{\prime}\right)_{m},(\mathbf{I I})_{m}$ and (7.10) imply that we can apply Lemma 7.1 for $m=1, \ldots, j-1$. Recalling the definition of $c_{4}(M)$ we calculate

$$
\begin{aligned}
\widetilde{\Psi}_{2}\left(\vartheta^{j} \varrho\right) & \leqslant \vartheta^{2 j \alpha} \widetilde{\Psi}_{2}(\varrho)+c_{3}(2 M)\left(\vartheta^{j} \varrho\right)^{2 \beta} \vartheta^{-2 \beta} \sum_{m=0}^{j-1} \vartheta^{2(\alpha-\beta) m} \leqslant \vartheta^{2 j \alpha} \widetilde{\Psi}_{2}(\varrho)+\frac{c_{3}(2 M)}{\vartheta^{2 \beta}-\vartheta^{2 \alpha}}\left(\vartheta^{j} \varrho\right)^{2 \beta} \\
& =\vartheta^{2 j \alpha} \widetilde{\Psi}_{2}(\varrho)+c_{4}(M)\left(\vartheta^{j} \varrho\right)^{2 \beta}
\end{aligned}
$$

showing $(\mathbf{I})_{j}$. To show $(\mathbf{I I})_{j}$ we estimate

$$
\begin{aligned}
& \left|\ell_{\vartheta^{j} \varrho}\left(z_{0}\right)\right|+\left|D \ell_{\vartheta^{j} \varrho}\right| \\
& \leqslant\left|\ell_{\varrho}\left(z_{0}\right)\right|+\left|D \ell_{\varrho}\right|+\sum_{m=1}^{j}\left|\ell_{\vartheta^{m} \varrho}\left(z_{0}\right)-\ell_{\vartheta^{m-1} \varrho}\left(z_{0}\right)\right|+\sum_{m=1}^{j}\left|D \ell_{\vartheta^{m} \varrho}-D \ell_{\vartheta^{m-1} \varrho}\right| \\
& \leqslant M+\sum_{m=1}^{j}\left[f_{Q_{\vartheta^{m} \varrho}\left(z_{0}\right)}\left|u-\ell_{\vartheta^{m-1}}\right|^{2} \mathrm{~d} z\right]^{1 / 2}+\sum_{m=1}^{j}\left[\frac{n(n+2)}{\left(\vartheta^{m} \varrho\right)^{2}} f_{Q_{\vartheta^{m}}\left(z_{0}\right)}\left|u-\ell_{\vartheta^{m-1} \varrho}\right|^{2} \mathrm{~d} z\right]^{1 / 2} \\
& \leqslant M+\frac{1+\sqrt{n(n+2)}}{\sqrt{\vartheta^{n+4}}} \sum_{m=1}^{j} \Psi_{2}\left(\vartheta^{m-1} \varrho\right)^{1 / 2} \leqslant M+\frac{n+2}{\sqrt{\vartheta^{n+4}}} \sum_{m=0}^{j-1} \sqrt{\vartheta^{2 m \alpha} \widetilde{\Psi}_{2}(\varrho)+c_{4}(M)\left(\vartheta^{m} \varrho\right)^{2 \beta}}
\end{aligned}
$$




$$
\leqslant M+\frac{(n+2)}{\sqrt{\vartheta^{n+4}}}\left(\frac{\sqrt{\widetilde{\Psi}_{2}(\varrho)}}{1-\vartheta^{\alpha}}+\frac{\sqrt{c_{4}(M) \varrho^{2 \beta}}}{1-\vartheta^{\beta}}\right) \leqslant M+\frac{M}{2}+\frac{M}{2}=2 M .
$$

Here we have used in turn Lemma 3.1, the definition of $\Psi_{2}\left(\vartheta^{m-1} \varrho\right),(\mathbf{I})_{m}$ for $m=1, \ldots, j-1,(7.11)$ and (7.12).

The above reasoning proves the first assertion of the following

Lemma 7.3. For $M>1$ and $Q_{\varrho}\left(z_{0}\right) \Subset Q_{T}$, suppose that the conditions

(i) $\left|\ell_{\varrho}\left(z_{0}\right)\right|+\left|D \ell_{\varrho}\right| \leqslant M$,

(ii) $\varrho \leqslant \varrho_{0}(M)$,

(iii) $\widetilde{\Psi}_{2}(\varrho) \leqslant \widetilde{\Psi}_{0}(M)$,

are satisfied. Then for every $j \in \mathbb{N}$ we have

$$
\widetilde{\Psi}_{2}\left(\vartheta^{j} \varrho\right) \leqslant \vartheta^{2 \alpha j} \widetilde{\Psi}_{2}(\varrho)+c_{4}(M)\left(\vartheta^{j} \varrho\right)^{2 \beta} \text { and }\left|\ell_{\vartheta^{j} \varrho}\left(z_{0}\right)\right|+\left|D \ell_{\vartheta^{j} \varrho}\right| \leqslant 2 M .
$$

Moreover, the limit

$$
\Gamma_{z_{0}}:=\lim _{j \rightarrow \infty}(D u)_{z_{0}, \vartheta^{j} \varrho / 2}
$$

exists, and the estimate

$$
f_{Q_{r}\left(z_{0}\right)}\left|D u-\Gamma_{z_{0}}\right|^{2} \mathrm{~d} z \leqslant c\left[\left(\frac{r}{\varrho / 2}\right)^{2 \alpha} \Psi_{2}(\varrho)+r^{2 \beta}\right]
$$

is valid for $0<r \leqslant \varrho / 2$ for a constant $c=c(n, N, \lambda, \alpha, L, \beta, M)$.

Proof. Since $\left|D \ell_{\vartheta^{j}}{ }\right| \leqslant 2 M$ we are in a position to apply Lemma 5.1. We obtain

$$
\begin{aligned}
\Phi_{2}\left(\vartheta^{j} \varrho / 2,(D u)_{\vartheta^{j} \varrho / 2}\right) & \leqslant \Phi_{2}\left(\vartheta^{j} \varrho / 2,(D \ell)_{\vartheta^{j} \varrho}\right) \leqslant c_{\text {Cacc }}(2 M) \widetilde{\Psi}_{2}\left(\vartheta^{j} \varrho\right) \\
& \leqslant c_{\text {Cacc }}(2 M)\left(\vartheta^{2 \alpha j} \widetilde{\Psi}_{2}(\varrho)+c_{4}(M)\left(\vartheta^{j} \varrho\right)^{2 \beta}\right) .
\end{aligned}
$$

We now consider $0<r \leqslant \varrho / 2$. We fix $k \in \mathbb{N} \cup\{0\}$ with $\vartheta^{k+1} \varrho / 2<r \leqslant \vartheta^{k} \varrho / 2$. Then the previous estimate implies

$$
\begin{aligned}
\Phi_{2}\left(r,(D u)_{r}\right) & =\underset{Q_{r}\left(z_{0}\right)}{f}\left|D u-(D u)_{r}\right|^{2} \mathrm{~d} z \leqslant \vartheta^{-n-2} \underset{Q_{\vartheta^{k} \varrho / 2}\left(z_{0}\right)}{f}\left|D u-(D u)_{\vartheta^{k} \varrho / 2}\right|^{2} \mathrm{~d} z \\
& \leqslant \vartheta^{-n-2} c_{\mathrm{Cacc}}(2 M)\left[\vartheta^{2 \alpha k} \widetilde{\Psi}_{2}(\varrho)+c_{4}(M)\left(\vartheta^{k} \varrho\right)^{2 \beta}\right] \\
& \leqslant \vartheta^{-n-2} c_{\mathrm{Cacc}}(2 M)\left[\vartheta^{-2 \alpha}\left(\frac{r}{\varrho / 2}\right)^{2 \alpha} \widetilde{\Psi}_{2}(\varrho)+c_{4}(M) 2^{2 \beta} \vartheta^{-2 \beta} r^{2 \beta}\right] \\
& \leqslant 4 \vartheta^{-n-2-2 \alpha} c_{\mathrm{Cacc}}(2 M)\left[\left(\frac{r}{\varrho / 2}\right)^{2 \alpha} \Psi_{2}(\varrho)+\left(c_{4}(M)+1\right) r^{2 \beta}\right] \\
& \leqslant c_{\mathrm{dec}}(M)\left[\left(\frac{r}{\varrho / 2}\right)^{2 \alpha} \Psi_{2}(\varrho)+r^{2 \beta}\right] .
\end{aligned}
$$

Next, we show that $\left((D u)_{\vartheta^{j} \varrho / 2}\right)_{j \in \mathbb{N}}$ is a Cauchy sequence in $\mathbb{R}^{n N}$. For $k>j$ we deduce 


$$
\begin{aligned}
\left|(D u)_{\vartheta^{j} \varrho / 2}-(D u)_{\vartheta^{k} \varrho / 2}\right| & \leqslant \sum_{m=j+1}^{k}\left|(D u)_{\vartheta^{m} \varrho / 2}-(D u)_{\vartheta^{m-1} \varrho / 2}\right| \\
& \leqslant \sqrt{\vartheta^{-n-2}} \sum_{m=j}^{k-1}\left[f_{Q_{\vartheta^{m} \varrho / 2}\left(z_{0}\right)}\left|D u-(D u)_{\vartheta^{m} \varrho / 2}\right|^{2} \mathrm{~d} z\right]^{1 / 2} \\
& =\sqrt{\vartheta^{-n-2}} \sum_{m=j}^{k-1} \sqrt{\Phi_{2}\left(\vartheta^{m} \varrho / 2\right)} \\
& \leqslant \sqrt{\vartheta^{-n-2} c_{\operatorname{Cacc}}(2 M)} \sum_{m=j}^{k-1} \sqrt{\vartheta^{2 \alpha m} \widetilde{\Psi}_{2}(\varrho)+c_{4}(M)\left(\vartheta^{m} \varrho\right)^{2 \beta}} \\
& \leqslant \sqrt{\vartheta^{-n-2} c_{\operatorname{Cacc}}(2 M)}\left(\frac{\sqrt{\widetilde{\Psi}_{2}(\varrho)}}{1-\vartheta^{\alpha}} \vartheta^{\alpha j}+\frac{\sqrt{c_{4}(M) \varrho^{2 \beta}}}{1-\vartheta^{\beta}} \vartheta^{\beta j}\right) .
\end{aligned}
$$

This proves the claim. Therefore the limit $\Gamma_{z_{0}}=\lim _{j \rightarrow \infty}(D u)_{\vartheta^{j} \varrho / 2} \in \mathbb{R}^{n N}$ exists and from the previous estimate we infer (taking the limit $k \rightarrow \infty$ )

$$
\left|(D u)_{\vartheta^{j} \varrho / 2}-\Gamma_{z_{0}}\right| \leqslant c_{5}(M) \sqrt{\vartheta^{2 \alpha j} \widetilde{\Psi}_{2}(\varrho)+\left(\vartheta^{j} \varrho\right)^{2 \beta}} .
$$

Combining this with (7.13) we arrive at

$$
f_{Q_{\vartheta^{j} \varrho / 2}\left(z_{0}\right)}\left|D u-\Gamma_{z_{0}}\right|^{2} \mathrm{~d} z \leqslant 2 \Phi_{2}\left(\vartheta^{j} \varrho / 2\right)+2\left|(D u)_{\vartheta^{j} \varrho / 2}-\Gamma_{z_{0}}\right|^{2} \leqslant c(M)\left(\vartheta^{2 \alpha j} \widetilde{\Psi}_{2}(\varrho)+\left(\vartheta^{j} \varrho\right)^{2 \beta}\right) .
$$

For $0<r \leqslant \varrho / 2$ we find $k \in \mathbb{N} \cup\{0\}$ with $\vartheta^{k+1} \varrho / 2<r \leqslant \vartheta^{k} \varrho / 2$. Then the previous estimate implies

$$
\begin{aligned}
f_{Q_{r}\left(z_{0}\right)}\left|D u-\Gamma_{z_{0}}\right|^{2} \mathrm{~d} z & \leqslant \vartheta^{-n-2} \int_{Q_{\vartheta^{k}}\left(z_{0}\right)}\left|D u-\Gamma_{z_{0}}\right|^{2} \mathrm{~d} z \leqslant \vartheta^{-n-2} c(M)\left[\vartheta^{2 \alpha k} \widetilde{\Psi}_{2}(\varrho)+\left(\vartheta^{k} \varrho\right)^{2 \beta}\right] \\
& \leqslant c(M)\left[\left(\frac{r}{\varrho / 2}\right)^{2 \alpha} \Psi_{2}(\varrho)+r^{2 \beta}\right] .
\end{aligned}
$$

This proves the assertion of the lemma.

Remark 7.4. Using Remark 7.2, a careful reading of the proof above yields that condition (i) in Lemma 7.3 can be relaxed to $\left|D \ell_{\varrho}\right| \leqslant M$.

An immediate consequence of the previous lemma and of the isomorphism theorem of Campanato-Da Prato [6] is the first regularity result of the paper:

Theorem 7.5 (Description of regular points). Let $u \in L^{2}\left(-T, 0 ; W^{1,2}\left(\Omega, \mathbb{R}^{N}\right)\right)$ be a weak solution to the system (1.1) under the assumptions (2.1)-(2.3) and (2.6) and denote by $\Sigma$ the singular set of $u$ (as explained in Section 1). Then

$$
\Sigma \subset \Sigma_{0} \cup \Sigma_{2},
$$

where 


$$
\begin{aligned}
& \Sigma_{0}:=\left\{z_{0} \in Q_{T}: \liminf _{\varrho \downarrow 0} \varrho^{-2} \underset{Q_{\varrho}\left(z_{0}\right)}{f}\left|u-(u)_{z_{0}, \varrho}-(D u)_{z_{0}, \varrho}\left(x-x_{0}\right)\right|^{2} \mathrm{~d} z>0\right\}, \\
& \Sigma_{2}:=\left\{z_{0} \in Q_{T}: \limsup _{\varrho \downarrow 0}\left(\left|(u)_{z_{0}, \varrho}\right|+\left|(D u)_{z_{0}, \varrho}\right|\right)=\infty\right\} .
\end{aligned}
$$

Remark 7.6. Remark 7.4 gives that for systems of the type (1.4) the set $\Sigma_{2}$ above can be replaced by the smaller

$$
\widetilde{\Sigma}_{2}:=\left\{z_{0} \in Q_{T}: \underset{\varrho \downarrow 0}{\limsup }\left|(D u)_{z_{0}, \varrho}\right|=\infty\right\} .
$$

Now it happens that while $\left|\Sigma_{2}\right|=0$ and $\left|\widetilde{\Sigma}_{2}\right|=0$ by Lebesgue theory, we cannot a-priori assert the same about $\Sigma_{0}$, since the function $u$ is not assumed to be differentiable with respect to time. Therefore the previous results only characterize the regular points but is not a partial regularity result in the sense that it does not immediately imply that $|\Sigma|=0$. For this, we still need another section.

\section{Partial regularity}

In this section we finally prove Theorem 1.2 ; this is a consequence of the following:

Theorem 8.1 (Almost everywhere regularity). Let $u \in L^{2}\left(-T, 0 ; W^{1,2}\left(\Omega, \mathbb{R}^{N}\right)\right)$ be a weak solution to the system (1.1) under the assumptions (2.1)-(2.3) and (2.5) and denote by $\Sigma$ the singular set of $u$; then

$$
\Sigma \subset \Sigma_{1} \cup \Sigma_{2},
$$

where $\Sigma_{2}$ is as in Theorem 7.5 and

$$
\Sigma_{1}:=\left\{z_{0} \in Q_{T}: \liminf _{\varrho \downarrow 0} f_{Q_{\varrho}\left(z_{0}\right)}\left|D u-(D u)_{z_{0}, \varrho}\right|^{2} \mathrm{~d} z>0\right\} \cup\left\{z_{0} \in Q_{T}: \liminf _{\varrho \downarrow 0} f_{Q_{\varrho}\left(z_{0}\right)}\left|u-(u)_{z_{0}, \varrho}\right|^{2} \mathrm{~d} z>0\right\} .
$$

Proof. We start taking a point $z_{0} \equiv\left(x_{0}, t_{0}\right) \in Q_{T}$ such that

$$
\liminf _{\varrho \downarrow 0} f_{Q_{\varrho}\left(z_{0}\right)}\left|D u-(D u)_{z_{0}, \varrho}\right|^{2} \mathrm{~d} z=0, \quad \liminf _{\varrho \downarrow 0} f_{Q_{\varrho}\left(z_{0}\right)}\left|u-(u)_{z_{0}, \varrho}\right|^{2} \mathrm{~d} z=0
$$

and

$$
\sup _{\varrho>0}\left|(u)_{z_{0}, \varrho}\right|+\sup _{\varrho>0}\left|(D u)_{z_{0}, \varrho}\right| \leqslant M<\infty .
$$

The proof is complete if we show that such points are a regular points.

Step 1: a comparison estimate. The main goal here is to achieve the estimates (8.5), (8.6) below. The following argument can be justified by the use of Steklov-averages; we shall omit all the details, only proceeding formally. Consider the unique weak solution $v \in L^{2}\left(t_{0}-4 \varrho^{2}, t_{0} ; W^{1,2}\left(B_{2 \varrho}\left(x_{0}\right), \mathbb{R}^{N}\right)\right)$ of the initial boundary value problem

$$
\left\{\begin{array}{l}
\int_{Q_{2 \varrho}\left(z_{0}\right)}\left(v \varphi_{t}-A\left(z_{0},(u)_{z_{0}, 2 \varrho}, D v\right) D \varphi\right) \mathrm{d} z=0 \quad \forall \varphi \in C_{0}^{\infty}\left(Q_{2 \varrho}\left(z_{0}\right), \mathbb{R}^{N}\right), \\
v=u \quad \text { on } B_{2 \varrho}\left(x_{0}\right) \times\left\{t_{0}-4 \varrho^{2}\right\} \cap \partial B_{2 \varrho}\left(x_{0}\right) \times\left(t_{0}-4 \varrho^{2}, t_{0}\right) .
\end{array}\right.
$$


Then the difference $u-v$ satisfies

$$
\int_{Q_{2 \varrho}\left(z_{0}\right)}\left((u-v) \varphi_{t}-\left(A(z, u, D u)-A\left(z_{0},(u)_{z_{0}, 2 \varrho}, D v\right)\right) D \varphi\right) \mathrm{d} z=0,
$$

for every $\varphi \in C_{0}^{\infty}\left(Q_{2 \varrho}\left(z_{0}\right), \mathbb{R}^{N}\right)$. We now choose $\varphi:=\chi(t)(u-v)$ with $\chi \equiv 1$ for $(-\infty, s), \chi \equiv 0$ on $(s+\varepsilon, \infty)$, and $\chi(t)=(s+\varepsilon-t) / \varepsilon$ for $s \leqslant t \leqslant s+\varepsilon$, where $[s, s+\varepsilon] \in\left(t_{0}-4 \varrho^{2}, t_{0}\right)$. Then we have

$$
\begin{aligned}
& \frac{1}{2} \int_{Q_{2 \varrho}\left(z_{0}\right)} \partial_{t}\left(|u-v|^{2} \chi\right) \mathrm{d} z+\frac{1}{2} \int_{Q_{2 \varrho}\left(z_{0}\right)}|u-v|^{2} \partial_{t} \chi \mathrm{d} z \\
& \quad-\int_{Q_{2 \varrho}\left(z_{0}\right)}\left(A(z, u, D u)-A\left(z_{0},(u)_{z_{0}, 2 \varrho}, D v\right)\right)(D u-D v) \chi \mathrm{d} z=0 .
\end{aligned}
$$

Letting $\varepsilon \downarrow 0$ we easily obtain that for a.e. $s \in\left(t_{0}-4 \varrho^{2}, t_{0}\right)$

$$
\begin{aligned}
& \frac{1}{2}\|u(\cdot, s)-v(\cdot, s)\|_{L^{2}\left(B_{2 \varrho}\left(x_{0}\right)\right)}^{2}+\int_{B_{2 \varrho}\left(x_{0}\right) \times\left(t_{0}-4 \varrho^{2}, s\right)}\left(A\left(z_{0},(u)_{z_{0}, 2 \varrho}, D u\right)-A\left(z_{0},(u)_{z_{0}, 2 \varrho}, D v\right)\right) D(u-v) \mathrm{d} z \\
& \quad=\int_{B_{2 \varrho}\left(x_{0}\right) \times\left(t_{0}-4 \varrho^{2}, s\right)}\left(A\left(z_{0},(u)_{z_{0}, 2 \varrho}, D u\right)-A(z, u, D u)\right) D(u-v) \mathrm{d} z .
\end{aligned}
$$

The second term of the left-hand side of the previous equation can be estimated by the use of monotonicity, i.e. $\left(A\left(z_{0},(u)_{z_{0}, 2 \varrho}, D u\right)-A\left(z_{0},(u)_{z_{0}, 2 \varrho}, D v\right)\right) D(u-v) \geqslant \lambda|D u-D v|^{2}$. We therefore obtain

$$
\begin{aligned}
& \frac{1}{2}\|u(\cdot, t)-v(\cdot, t)\|_{L^{2}\left(B_{2 \varrho}\left(x_{0}\right)\right)}^{2}+\lambda \int_{B_{2 \varrho}\left(x_{0}\right) \times\left(t_{0}-4 \varrho^{2}, t\right)}|D u-D v|^{2} \mathrm{~d} z \\
& \quad \leqslant \int_{B_{2 \varrho}\left(x_{0}\right) \times\left(t_{0}-4 \varrho^{2}, s\right)}\left(A\left(z_{0},(u)_{z_{0}, 2 \varrho}, D u\right)-A(z, u, D u)\right) D(u-v) \mathrm{d} z=I .
\end{aligned}
$$

To estimate the right-hand side we use (2.5) which easily yields

$$
\left|A\left(z_{0},(u)_{z_{0}, 2 \varrho}, D u\right)-A(z, u, D u)\right| \leqslant L \theta\left(2\left|(u)_{z_{0}, 2 \varrho}\right|+\left|u-(u)_{z_{0}, 2 \varrho}\right|, 4 \varrho+\left|u-(u)_{z_{0}, 2 \varrho}\right|\right)(1+|D u|) .
$$

Using the previous estimate, Young's inequality and the fact that $\theta \leqslant 1$, we have

$$
\begin{aligned}
|I| \leqslant & \frac{\lambda}{2} \int_{B_{2 \varrho}\left(x_{0}\right) \times\left(t_{0}-4 \varrho^{2}, s\right)}|D u-D v|^{2} \mathrm{~d} z \\
& +\frac{2 L^{2}}{\lambda} \int_{Q_{2 \varrho}\left(z_{0}\right)} \theta\left(\left|2(u)_{z_{0}, 2 \varrho}\right|+\left|u-(u)_{z_{0}, 2 \varrho}\right|, 4 \varrho+\left|u-(u)_{z_{0}, 2 \varrho}\right|\right)\left(1+|D u|^{2}\right) \mathrm{d} z .
\end{aligned}
$$

Absorbing the term $\frac{\lambda}{2} \int_{B_{2 \varrho}\left(x_{0}\right) \times\left(t_{0}-4 \varrho^{2}, s\right)}|D u-D v|^{2} \mathrm{~d} z$ on the left-hand side we arrive at

$$
\begin{aligned}
& \frac{1}{2}\|u(\cdot, t)-v(\cdot, t)\|_{L^{2}\left(B_{2 \varrho}\left(x_{0}\right)\right)}^{2}+\frac{\lambda}{2} \int_{B_{2 \varrho}\left(x_{0}\right) \times\left(t_{0}-4 \varrho^{2}, t\right)}|D u-D v|^{2} \mathrm{~d} z \\
& \quad \leqslant \frac{4 L^{2}}{\lambda} \int_{Q_{2 \varrho}\left(z_{0}\right)} \theta\left(\left|2(u)_{z_{0}, 2 \varrho}\right|+\left|u-(u)_{z_{0}, 2 \varrho}\right|, 4 \varrho+\left|u-(u)_{z_{0}, 2 \varrho}\right|\right)\left(1+|D u|^{2}\right) \mathrm{d} z=: \frac{4 L^{2}}{\lambda} I I .
\end{aligned}
$$


We shall provide an estimate for $I I$. We denote

$$
\varepsilon_{\sigma}:=\int_{Q_{\sigma}\left(z_{0}\right)}\left|u-(u)_{z_{0}, \sigma}\right| \mathrm{d} z, \quad \sigma>0 .
$$

If we let $A_{t}^{\varrho} \equiv A_{t}:=\left\{z \in Q_{2 \varrho}\left(z_{0}\right):\left|u-(u)_{z_{0}, 2 \varrho}\right| \geqslant t\right\}$ then

$$
\left|A_{t}\right| \leqslant \frac{1}{t} \int_{Q_{2 \varrho}\left(z_{0}\right)}\left|u-(u)_{z_{0}, 2 \varrho}\right| \mathrm{d} z \leqslant \frac{\left|Q_{2 \varrho}\right|}{t} \varepsilon_{2 \varrho} .
$$

We now split $I I$

$$
I I=\int_{A_{t}}(\ldots) \mathrm{d} z+\int_{Q_{2 \varrho}\left(z_{0}\right) \backslash A_{t}}(\ldots) \mathrm{d} z=: I I I+I V,
$$

and estimate $I I I$ and $I V$. We have, using that $\theta \leqslant 1$, (8.4) and (8.2)

$$
\begin{aligned}
I I I & \leqslant \int_{A_{t}}\left(1+|D u|^{2}\right) \mathrm{d} z \leqslant 2 \int_{Q_{2 \varrho}\left(z_{0}\right)}\left|D u-(D u)_{z_{0}, 2 \varrho}\right|^{2} \mathrm{~d} z+\left(1+2\left|(D u)_{z_{0}, 2 \varrho}\right|^{2}\right)\left|A_{t}\right| \\
& \leqslant 2 \int_{Q_{2 \varrho}\left(z_{0}\right)}\left|D u-(D u)_{z_{0}, 2 \varrho}\right|^{2} \mathrm{~d} z+2\left(1+2\left|(D u)_{z_{0}, 2 \varrho}\right|^{2}\right) \frac{\left|Q_{2 \varrho}\right|}{t} \varepsilon_{2 \varrho} \\
& \leqslant 2 \int_{Q_{2 \varrho}\left(z_{0}\right)}\left|D u-(D u)_{z_{0}, 2 \varrho}\right|^{2} \mathrm{~d} z+2\left(1+2 M^{2}\right) \frac{\left|Q_{2 \varrho}\right|}{t} \varepsilon_{2 \varrho} .
\end{aligned}
$$

From the definition of $\theta$ (Section 2) we have

$$
I V \leqslant 4 K(2 M+t)(\varrho+t)^{\beta} \int_{Q_{2 \varrho}\left(z_{0}\right)}\left(1+|D u|^{2}\right) \mathrm{d} z .
$$

We now choose the parameter $t$ carefully, i.e.

$$
t:=\sqrt{\varepsilon_{2 \varrho}} .
$$

Connecting the previous estimates for $I I, I I I$ and $I V$ to (8.3), we easily have the estimate we were interested in; that is

$$
\begin{aligned}
& f_{Q_{2 \varrho}\left(z_{0}\right)}|D u-D v|^{2} \mathrm{~d} z+\sup _{t_{0}-4 \varrho^{2} \leqslant t \leqslant t_{0}} \varrho^{-2} \underset{B_{2 \varrho}\left(z_{0}\right)}{f}|u(x, t)-v(x, t)|^{2} \mathrm{~d} x \\
& \leqslant c(\lambda, L) K\left(2 M+\sqrt{\varepsilon_{2 \varrho}}\right)\left(\varrho+\sqrt{\varepsilon_{2 \varrho}}\right)^{\beta} f_{Q_{2 \varrho}\left(z_{0}\right)}\left(1+|D u|^{2}\right) \mathrm{d} z \\
& \quad+\quad f_{Q_{2 \varrho}\left(z_{0}\right)}\left|D u-(D u)_{z_{0}, 2 \varrho}\right|^{2} \mathrm{~d} z+\sqrt{\varepsilon_{2 \varrho}}\left(1+M^{2}\right) \\
& \leqslant c\left[K\left(2 M+\sqrt{\varepsilon_{2 \varrho}}\right)\left(\varrho+\sqrt{\varepsilon_{2 \varrho}}\right)^{\beta}+1\right] f_{Q_{2 \varrho}\left(z_{0}\right)}\left|D u-(D u)_{z_{0}, 2 \varrho}\right|^{2} \mathrm{~d} z \\
& \quad+c\left[K\left(2 M+\sqrt{\varepsilon_{2 \varrho}}\right)\left(\varrho+\sqrt{\varepsilon_{2 \varrho}}\right)^{\beta}+\sqrt{\varepsilon_{2 \varrho}}\right]\left(1+M^{2}\right)=: S(\varrho) .
\end{aligned}
$$


In particular, we see that

$$
\varrho^{-2} f_{Q_{2 \varrho}\left(z_{0}\right)}|u(z)-v(z)|^{2} \mathrm{~d} z \leqslant \sup _{t_{0}-4 \varrho^{2} \leqslant t \leqslant t_{0}} \varrho^{-2} f_{B_{2 \varrho}\left(z_{0}\right)}|u(x, t)-v(x, t)|^{2} \mathrm{~d} x \leqslant S(\varrho) .
$$

We observe that as a consequence of (8.1) and (8.2) we have that

$$
\liminf _{\varrho \downarrow 0} S(\varrho)=0 .
$$

Step 2: A Poincaré type inequality. Our aim is to derive (8.8). Let us define

$$
\tilde{v}:=v-(D v)_{z_{0}, 2 \varrho}\left(x-x_{0}\right) .
$$

Therefore $\tilde{v}$ solves

$$
\int_{Q_{2 \varrho}\left(z_{0}\right)}\left(\tilde{v} \varphi_{t}-\tilde{A}(D \tilde{v}) D \varphi\right) \mathrm{d} z=0 \quad \forall \varphi \in C_{0}^{\infty}\left(Q_{2 \varrho}\left(z_{0}\right), \mathbb{R}^{N}\right),
$$

where $\tilde{A}(p):=A\left(z_{0},(u)_{z_{0}, 2 \varrho},(D v)_{z_{0}, 2 \varrho}+p\right)$ for every $p \in \mathbb{R}^{n N}$. By (2.2) and (2.3) we have

$$
\left|\frac{\partial \tilde{A}}{\partial p}(p)\right| \leqslant L, \quad \frac{\partial \tilde{A}}{\partial p}(p)(\tilde{p}, \tilde{p}) \geqslant \lambda|\tilde{p}|^{2}, \quad \forall p, \tilde{p} \in \mathbb{R}^{n N} .
$$

The last properties allow us to apply the relevant regularity theory for systems without coefficients of the type $u_{t}-\operatorname{div} \tilde{A}(D u)=0$ : from [5], Theorem 3.1 we conclude that $\tilde{v} \in W^{1,2}\left(t_{0}-\varrho^{2}, t_{0} ; W^{1,2}\left(B_{\varrho}\left(x_{0}\right), \mathbb{R}^{N}\right)\right)$ and that

$$
\begin{aligned}
\varrho^{2} f_{Q_{\varrho}\left(z_{0}\right)}\left|\partial_{t} v\right|^{2} \mathrm{~d} z & =\varrho^{2} f_{Q_{\varrho}\left(z_{0}\right)}\left|\partial_{t} \tilde{v}\right|^{2} \mathrm{~d} z \leqslant c(\lambda, L) \underset{Q_{2 \varrho}\left(z_{0}\right)}{f}|D \tilde{v}|^{2} \mathrm{~d} z=c(\lambda, L) \underset{Q_{2 \varrho}\left(z_{0}\right)}{f}\left|D v-(D v)_{z_{0}, 2 \varrho}\right|^{2} \mathrm{~d} z \\
& \leqslant c(\lambda, L)\left[f_{Q_{2 \varrho}\left(z_{0}\right)}|D u-D v|^{2} \mathrm{~d} z+f_{Q_{2 \varrho}\left(z_{0}\right)}\left|D u-(D u)_{z_{0}, 2 \varrho}\right|^{2} \mathrm{~d} z\right] .
\end{aligned}
$$

In view of the previous estimate, using Poincaré's inequality for $v$ and (8.5) we find

$$
\begin{aligned}
& \varrho^{-2} f_{Q_{\varrho}\left(z_{0}\right)}\left|v-(v)_{z_{0}, \varrho}-(D v)_{z_{0}, \varrho}\left(x-x_{0}\right)\right|^{2} \mathrm{~d} z \leqslant c\left[\underset{Q_{\varrho}\left(z_{0}\right)}{f}\left|D v-(D v)_{z_{0}, \varrho}\right|^{2} \mathrm{~d} z+\varrho^{2} \underset{Q_{\varrho}\left(z_{0}\right)}{f}\left|\partial_{t} v\right|^{2} \mathrm{~d} z\right] \\
& \quad \leqslant c\left[f_{Q_{2 \varrho}\left(z_{0}\right)}|D u-D v|^{2} \mathrm{~d} z+f_{Q_{2 \varrho}\left(z_{0}\right)}\left|D u-(D u)_{z_{0}, 2 \varrho}\right|^{2} \mathrm{~d} z\right] \leqslant c\left[S(\varrho)+f_{Q_{2 \varrho}\left(z_{0}\right)}\left|D u-(D u)_{z_{0}, 2 \varrho}\right|^{2} \mathrm{~d} z\right],
\end{aligned}
$$

where $c=c(n, \lambda, L)$. Finally, by comparison, we get the Poincaré inequality for $u$ via (8.5) and the previous estimate

$$
\begin{aligned}
& \varrho^{-2} \underset{Q_{\varrho}(0)}{f}\left|u-(u)_{z_{0}, \varrho}-(D u)_{z_{0}, \varrho}\left(x-x_{0}\right)\right|^{2} \mathrm{~d} z \\
& \quad \leqslant c\left[\varrho^{-2} f_{Q_{\varrho}\left(z_{0}\right)}|u-v|^{2} \mathrm{~d} z+f_{Q_{\varrho}\left(z_{0}\right)}|D u-D v|^{2} \mathrm{~d} z+\varrho^{-2} f_{Q_{\varrho}\left(z_{0}\right)}\left|v-(v)_{z_{0}, \varrho}-(D v)_{z_{0}, \varrho}\left(x-x_{0}\right)\right|^{2} \mathrm{~d} z\right] \\
& \quad \leqslant c\left[S(\varrho)+f_{Q_{2 \varrho}\left(z_{0}\right)}\left|D u-(D u)_{z_{0}, 2 \varrho}\right|^{2} \mathrm{~d} z\right]
\end{aligned}
$$

for a constant $c=c(n, \lambda, L)$. 
Step 3: Conclusion. From the previous estimate and (8.7) the assertion readily follows. Indeed if $z_{0} \in Q_{T}$ satisfies (8.1) and (8.2) then we have

$$
\liminf _{\varrho \downarrow 0} \varrho^{-2} f_{Q_{\varrho}(0)}\left|u-(u)_{z_{0}, \varrho}-(D u)_{z_{0} \varrho}\left(x-x_{0}\right)\right|^{2} \mathrm{~d} z=0
$$

therefore $z_{0}$ is a regular point in view of Theorem 7.5.

Theorem 8.2 (Almost everywhere regularity for simpler systems). Let $u \in L^{2}\left(-T, 0 ; W^{1,2}\left(\Omega, \mathbb{R}^{N}\right)\right)$ be a weak solution to the system (1.4) under the assumptions (2.1)-(2.3) and (2.8) and denote by $\Sigma$ the singular set of $u$; then

$$
\Sigma \subset \widetilde{\Sigma}_{1} \cup \widetilde{\Sigma}_{2}
$$

where $\widetilde{\Sigma}_{2}$ is as in Remark 7.6 and

$$
\widetilde{\Sigma}_{1}:=\left\{z_{0} \in Q_{T}: \liminf _{\varrho \downarrow 0} f_{Q_{\varrho}\left(z_{0}\right)}\left|D u-(D u)_{z_{0}, \varrho}\right|^{2} \mathrm{~d} z>0\right\} .
$$

Proof. The proof is based on a simple re-reading of Theorem 8.1; indeed it suffices to start with a point $z_{0} \equiv$ $\left(x_{0}, t_{0}\right) \in Q_{T}$ such that

$$
\liminf _{\varrho \downarrow 0} f_{Q_{\varrho}\left(z_{0}\right)}\left|D u-(D u)_{z_{0}, \varrho}\right|^{2} \mathrm{~d} z=0 \quad \text { and } \quad \sup _{\varrho>0}\left|(D u)_{z_{0}, \varrho}\right| \leqslant M<\infty .
$$

Since the vector field $A$ does not depend on $u$ the estimates in Step 1 simplify, especially those for II. In particular, in the definition of $S(\varrho)$ in (8.5) we can take $\varepsilon_{\varrho} \equiv 0$, therefore (8.9) suffices to have $S(\varrho) \rightarrow 0$, which is the fundamental information to use the estimates (8.5) and (8.6). The remaining Steps 2 and 3 do not need any adjustment.

\section{Proof of Theorem 1.3}

For $f \in L^{1}\left(Q_{T}\right)$ and $0<h<T$ we recall the definition of Steklov averages: $f_{h}$ of $f$ are defined for all $-T<t<0$ by

$$
f_{h}(x, t):= \begin{cases}\frac{1}{h} \int_{t}^{t+h} f(x, s) \mathrm{d} s, & t \in(-T,-h), \\ 0, & t>-h,\end{cases}
$$

respectively

$$
f_{\bar{h}}(x, t):= \begin{cases}\frac{1}{h} \int_{t-h}^{t} f(x, s) \mathrm{d} s, & t \in(-T+h, 0), \\ 0, & t<-T+h .\end{cases}
$$

For the properties of the Steklov averages we refer for instance to $[9,27]$. The following relations will be particularly useful in the sequel: For a.e. $(x, t) \in \Omega \times(-T,-h)$ and for a.e. $(x, t) \in \Omega \times(-T+h, 0)$, we have

$$
\frac{\partial f_{h}}{\partial t}=\frac{1}{h}(f(x, t+h)-f(x, t)), \quad \frac{\partial f_{\bar{h}}}{\partial t}=\frac{1}{h}(f(x, t)-f(x, t-h)) .
$$


Finally, for $|h|>0$ and $t \in(-T-|h|,-|h|)$ we define

$$
\tau_{h}(f) \equiv \tau_{h} f \equiv\left(\tau_{h} f\right)(x, t):=f(x, t+h)-f(x, t) .
$$

The following lemma asserts a well-known property of the time derivative that we restate in the way it is required later.

Lemma 9.1 (Fractional time derivative of $u$ ). Let $u \in L^{2}\left(-T, 0 ; W^{1,2}\left(\Omega, \mathbb{R}^{N}\right)\right)$ be a weak solution of the nonlinear parabolic system (1.1) under the only assumption $(2.1),\left(t_{0}, t_{1}\right) \Subset(-T, 0)$, and $\eta \in C_{0}^{\infty}(\Omega)$ a cut-off function with spt $\eta \Subset \Omega$. Then, whenever $0<|h|<1 / 2 \min \left\{\left|t_{1}\right|, T-\left|t_{0}\right|, 1\right\}$ the following estimate holds:

$$
\int_{t_{0}}^{t_{1}} \int_{\Omega} \eta^{2}|u(x, t+h)-u(x, t)|^{2} \mathrm{~d} x \mathrm{~d} t \leqslant c\left(\|\eta\|_{L^{\infty}}^{2}+\|\nabla \eta\|_{L^{\infty}}^{2}\right)|h| \int_{Q_{T}}\left(1+|D u|^{2}\right) \mathrm{d} z,
$$

where $c=c(L)$.

Proof. For brevity we shall only give the proof for the case $h>0$, the other one being the same, using $u_{\bar{h}}$ instead of $u_{h}$. Using Steklov averages to formulate (1.1) we have that for a.e. $t \in\left(t_{0}, t_{1}\right)$ and $h$ as above

$$
\int_{\Omega}\left(\partial_{t}\left(u_{h}\right) \varphi+[A(x, t, u, D u)]_{h} D \varphi\right) \mathrm{d} z=0, \quad \forall \varphi \in W_{0}^{1,2}\left(\Omega, \mathbb{R}^{N}\right) .
$$

Using the fact that $h \partial_{t}\left(u_{h}\right)=\tau_{h} u$ we get

$$
\int_{\Omega}\left(\frac{\tau_{h} u}{h} \varphi+[A(x, t, u, D u)]_{h} D \varphi\right) \mathrm{d} z=0 .
$$

We use the test function $\varphi=\eta^{2} \tau_{h} u$ and integrate with respect to $t$ over the interval $\left(t_{0}, t_{1}\right)$, obtaining

$$
\int_{t_{0}} \int_{\Omega}^{t_{1}} \frac{\left|\tau_{h} u\right|^{2}}{h} \eta^{2} \mathrm{~d} x \mathrm{~d} t=-\int_{t_{0}}^{t_{1}} \int_{\Omega}[A(x, t, u, D u)]_{h}\left[2 \eta \nabla \eta \otimes \tau_{h} u+\eta^{2} D \tau_{h} u\right] \mathrm{d} x \mathrm{~d} t=: I+I I .
$$

The first term is estimated using (2.1) and Young's inequality

$$
\begin{aligned}
|I| & \leqslant \frac{1}{2} \int_{t_{0}}^{t_{1}} \int_{\Omega} \frac{\left|\tau_{h} u\right|^{2}}{h} \eta^{2} \mathrm{~d} x \mathrm{~d} t+h \int_{t_{0}} \int_{\Omega}\left|[A(x, t, u, D u)]_{h}\right|^{2}|\nabla \eta|^{2} \mathrm{~d} x \mathrm{~d} t \\
& \leqslant \frac{1}{2} \int_{t_{0}}^{t_{1}} \int_{\Omega} \frac{\left|\tau_{h} u\right|^{2}}{h} \eta^{2} \mathrm{~d} x \mathrm{~d} t+L^{2}\|\nabla \eta\|_{L^{\infty}}^{2} h \int_{t_{0}}^{t_{1}}\left[(1+|D u|)^{2}\right]_{h} \mathrm{~d} x \mathrm{~d} t \\
& \leqslant \frac{1}{2} \int_{t_{0}}^{t_{1}} \int_{\Omega} \frac{\left|\tau_{h} u\right|^{2}}{h} \eta^{2} \mathrm{~d} x \mathrm{~d} t+L^{2}\|\nabla \eta\|_{L^{\infty}}^{2} h \int_{t_{0}}^{t_{1}+h} \int_{\Omega}(1+|D u|)^{2} \mathrm{~d} x \mathrm{~d} t \\
& \leqslant \frac{1}{2} \int_{t_{0}}^{t_{1}} \int_{\Omega} \frac{\left|\tau_{h} u\right|^{2}}{h} \eta^{2} \mathrm{~d} x \mathrm{~d} t+L^{2}\|\nabla \eta\|_{L^{\infty}}^{2} h \int_{Q_{T}}(1+|D u|)^{2} \mathrm{~d} z .
\end{aligned}
$$


Here we have used in the second-last line the elementary estimate

$$
\int_{t_{0}}^{t_{1}} \int_{\Omega}\left|f_{h}\right|^{2} \mathrm{~d} x \mathrm{~d} t \leqslant \int_{t_{0}}^{t_{1}+h} \int_{\Omega}|f|^{2} \mathrm{~d} x \mathrm{~d} t
$$

where $f \in L^{2}\left(Q_{T}\right),\left(t_{0}, t_{1}\right) \Subset(-T, 0)$ and $0<h<-t_{1}$. The second term is estimated similarly

$$
\begin{aligned}
|I I| & \leqslant\left(\int_{t_{0}}^{t_{1}} \int_{\Omega} \eta^{2}\left|[a(x, t, u, D u)]_{h}\right|^{2} \mathrm{~d} x \mathrm{~d} t\right)^{1 / 2}\left(\int_{t_{0}}^{t_{1}} \int_{\Omega} \eta^{2}\left|D \tau_{h} u\right|^{2} \mathrm{~d} x \mathrm{~d} t\right)^{1 / 2} \\
& \leqslant 2 L\left(\int_{t_{0}}^{t_{1}} \int_{\Omega} \eta^{2}\left[(1+|D u|)^{2}\right]_{h} \mathrm{~d} x \mathrm{~d} t\right)^{1 / 2}\left(\int_{t_{0}}^{t_{1}+h} \int_{\Omega} \eta^{2}|D u|^{2} \mathrm{~d} x \mathrm{~d} t\right)^{1 / 2} \\
& \leqslant 2 L\|\eta\|_{L^{\infty}}^{2} \int_{t_{0}}^{t_{1}+h}(1+|D u|)^{2} \mathrm{~d} x \mathrm{~d} t \leqslant 2 L\|\eta\|_{L^{\infty}}^{2} \int_{Q_{T}}(1+|D u|)^{2} \mathrm{~d} z
\end{aligned}
$$

while in the second line we have used the elementary estimate

$$
\int_{t_{0}}^{t_{1}} \int_{\Omega}\left|\tau_{h} f\right|^{2} \mathrm{~d} x \mathrm{~d} t \leqslant 2 \int_{t_{0}}^{t_{1}+h} \int_{\Omega}|f|^{2} \mathrm{~d} x \mathrm{~d} t
$$

Combining the estimates for $I$ and $I I$ with (9.1) we finally conclude with

$$
\int_{t_{0}}^{t_{1}} \int_{\Omega} \frac{\left|\tau_{h} u\right|^{2}}{h} \eta^{2} \mathrm{~d} x \mathrm{~d} t \leqslant c(L)\left(\|\eta\|_{L^{\infty}}^{2}+\|\nabla \eta\|_{L^{\infty}}^{2}\right) h \int_{Q_{T}}(1+|D u|)^{2} \mathrm{~d} z .
$$

As a consequence of the previous lemma we have

Corollary 9.2. Let $u \in L^{2}\left(-T, 0 ; W^{1,2}\left(\Omega, \mathbb{R}^{N}\right)\right)$ be a weak solution of the nonlinear parabolic system (1.1) under the only assumption $(2.1),\left(t_{0}, t_{1}\right) \Subset(-T, 0)$, and $\widetilde{\Omega} \Subset \Omega$. Then, whenever $0<|h|<1 / 2 \min \left(\left|t_{1}\right|, T-\left|t_{0}\right|, 1\right)$ the following estimate holds:

$$
\int_{t_{0}}^{t_{1}} \int_{\widetilde{\Omega}}|u(x, t+h)-u(x, t)|^{2} \mathrm{~d} x \mathrm{~d} t \leqslant c|h| \int_{Q_{T}}\left(1+|D u|^{2}\right) \mathrm{d} z,
$$

where $c=c(L, \operatorname{dist}(\widetilde{\Omega}, \partial \Omega))$.

In the following we state a preliminary estimate for the space time derivative of a weak solution $u$ of the nonlinear parabolic system (1.1) which we are going to use as a starting point; to be more precise we will estimate $\left|\tau_{h} D u\right|$. Let us fix $-T<\tilde{t}<t_{1}<0$, arbitrarily. In the following we shall always take

$$
|h| \leqslant \frac{1}{1000} \min \left\{\left|t_{1}\right|, T+t_{0}\right\}, \quad|\lambda| \leqslant \frac{1}{1000} \min \left\{\left|t_{1}\right|, T+t_{0}\right\}
$$

and $t \in\left(t_{0}, t_{1}\right)$ where $t_{0}:=(-T+\tilde{t}) / 2<\tilde{t}$. We shall fix a cut-off function in time $\zeta \in C^{\infty}((-T, 0))$ such that

$$
\zeta(t)=0 \quad \text { on }\left(-T, t_{0}\right), \quad \zeta(t)=1 \quad \text { for } t>\tilde{t}, \quad \zeta^{\prime} \geqslant 0, \quad\left|\zeta^{\prime}\right| \leqslant \frac{4}{T+t_{0}} .
$$


Moreover we choose a cut-off function in space $\eta \in C_{0}^{\infty}(\Omega)$ such that $|\operatorname{spt} \eta|>0$. We start once again from the Steklov-average formulation of (1.1)

$$
\int_{\Omega}\left(\partial_{t}\left(u_{\lambda}\right) \varphi+[A(x, t, u, D u)]_{\lambda} D \varphi\right) \mathrm{d} z=0, \quad \forall \varphi \in W_{0}^{1,2}\left(\Omega, \mathbb{R}^{N}\right) .
$$

Writing the previous equation at levels $t, t+h$ we get

$$
\int_{\Omega}\left(\partial_{t}\left(\tau_{h} u_{\lambda}\right) \varphi+\tau_{h}[A(x, t, u, D u)]_{\lambda} D \varphi\right) \mathrm{d} z=0 .
$$

Here we have used the identity $\tau_{h}\left(\partial_{t} u_{\lambda}\right)=\partial_{t}\left(\tau_{h} u_{\lambda}\right)$. In (9.6) we choose the test function

$$
\varphi(x, t):=\zeta^{2}(t) \eta^{2}(x)\left(\tau_{h} u_{\lambda}\right)(x, t) .
$$

It is easy to see that $\varphi$ is admissible in (9.6) for a.e. $t \in\left(t_{0}, t_{1}\right)$, i.e. that $\varphi(\cdot, t) \in W_{0}^{1,2}\left(\Omega, \mathbb{R}^{N}\right)$. Therefore taking $\varphi(\cdot, t)$ at each level $t$ and integrating over $\left(t_{0}, t_{1}\right)$ with respect to $t$ we obtain

$$
\int_{t_{0}}^{t_{1}} \int_{\Omega} \zeta^{2} \eta^{2} \partial_{t}\left(\tau_{h} u_{\lambda}\right)\left(\tau_{h} u_{\lambda}\right) \mathrm{d} x \mathrm{~d} t=-\int_{t_{0}}^{t_{1}} \int_{\Omega} \zeta^{2} \tau_{h}[A(x, t, u, D u)]_{\lambda}\left[2 \eta \nabla \eta \otimes\left(\tau_{h} u_{\lambda}\right)+\eta^{2} D\left(\tau_{h} u_{\lambda}\right)\right] \mathrm{d} x \mathrm{~d} t .
$$

We are now in a position to treat the left-hand side in a standard way. Recalling that $\zeta(t)=0$ for $-T<t \leqslant t_{0}$ we can rewrite the left-hand side of the previous identity in the form

$$
\begin{aligned}
\int_{t_{0}}^{t_{1}} \int_{\Omega} \zeta^{2} \eta^{2} \partial_{t}\left(\tau_{h} u_{\lambda}\right)\left(\tau_{h} u_{\lambda}\right) \mathrm{d} x \mathrm{~d} t & =\int_{-T}^{t_{1}} \int_{\Omega} \zeta^{2} \eta^{2} \partial_{t}\left(\tau_{h} u_{\lambda}\right)\left(\tau_{h} u_{\lambda}\right) \mathrm{d} x \mathrm{~d} t \\
& =\frac{1}{2} \int_{\Omega} \zeta^{2}\left(t_{1}\right) \eta^{2}\left|\tau_{h} u_{\lambda}\right|^{2}\left(\cdot, t_{1}\right) \mathrm{d} x-\int_{-T}^{t_{1}} \int_{\Omega} \zeta \zeta^{\prime} \eta^{2}\left|\tau_{h} u_{\lambda}\right|^{2} \mathrm{~d} x \mathrm{~d} t .
\end{aligned}
$$

Substituting this above and letting $\lambda \rightarrow 0$ we obtain for a.e. $t_{1}$ as above

$$
\begin{aligned}
& \frac{1}{2} \int_{\Omega} \zeta^{2}\left(t_{1}\right) \eta^{2}\left|\tau_{h} u\right|^{2}\left(\cdot, t_{1}\right) \mathrm{d} x-\int_{-T \Omega}^{t_{1}} \int_{\Omega} \zeta \zeta^{\prime} \eta^{2}\left|\tau_{h} u\right|^{2} \mathrm{~d} x \mathrm{~d} t \\
& \quad+\int_{t_{0}}^{t_{1}} \int_{\Omega} \zeta^{2} \tau_{h}[A(x, t, u, D u)]\left[2 \eta \nabla \eta \otimes\left(\tau_{h} u\right)+\eta^{2} D\left(\tau_{h} u\right)\right] \mathrm{d} x \mathrm{~d} t=0 .
\end{aligned}
$$

In the sequel the contribution of the first integral appearing in (9.7) will be ignored. We shall use the following decomposition of $\tau_{h}(A)$ :

$$
\begin{aligned}
\tau_{h}[ & A(\cdot, \cdot, u(\cdot, \cdot), D u(\cdot, \cdot))](x, t) \\
= & A(x, t+h, u(x, t+h), D u(x, t+h))-A(x, t, u(x, t), D u(x, t)) \\
= & A(x, t+h, u(x, t+h), D u(x, t+h))-A(x, t+h, u(x, t+h), D u(x, t)) \\
& +A(x, t+h, u(x, t+h), D u(x, t))-A(x, t+h, u(x, t), D u(x, t)) \\
& \quad+A(x, t+h, u(x, t), D u(x, t))-A(x, t, u(x, t), D u(x, t)) \\
= & : \mathcal{A}(h)+\mathcal{B}(h)+\mathcal{C}(h) .
\end{aligned}
$$


With such a notation (9.7) turns to

$$
\begin{aligned}
& \int_{t_{0}}^{t_{1}} \int_{\Omega} \zeta^{2} \eta^{2}[\mathcal{A}(h)+\mathcal{B}(h)+\mathcal{C}(h)] D\left(\tau_{h} u\right) \mathrm{d} x \mathrm{~d} t \\
& \quad \leqslant-2 \int_{t_{0}}^{t_{1}} \int_{\Omega} \zeta^{2}[\mathcal{A}(h)+\mathcal{B}(h)+\mathcal{C}(h)] \eta \nabla \eta \otimes\left(\tau_{h} u\right) \mathrm{d} x \mathrm{~d} t+\int_{-T \Omega}^{t_{1}} \int_{\Omega} \zeta \zeta^{\prime} \eta^{2}\left|\tau_{h} u\right|^{2} \mathrm{~d} x \mathrm{~d} t .
\end{aligned}
$$

We proceed estimating the various pieces arising in (9.8).

Estimates for integrals involving $\mathcal{A}(h)$. We write

$$
\mathcal{A}(h)(x, t)=\int_{0}^{1} \frac{\partial A}{\partial p}\left(x, t+h, u(x, t+h), D u(x, t)+s \tau_{h}(D u)(x, t)\right) \mathrm{d} s \tau_{h}(D u)(x, t)=: \tilde{\mathcal{A}}(h) \tau_{h}(D u)(x, t) .
$$

Using the ellipticity of $A$, i.e. (2.3) we see that

$$
\left\langle\mathcal{A}(h), \tau_{h}(D u)\right\rangle=\tilde{\mathcal{A}}(h) \tau_{h}(D u) \cdot \tau_{h}(D u) \geqslant \lambda\left|\tau_{h} D u\right|^{2} .
$$

Therefore we obtain

$$
\int_{t_{0}}^{t_{1}} \int_{\Omega} \zeta^{2} \eta^{2} \mathcal{A}(h) D\left(\tau_{h} u\right) \mathrm{d} x \mathrm{~d} t \geqslant \lambda \int_{t_{0}}^{t_{1}} \int_{\Omega} \zeta^{2} \eta^{2}\left|\tau_{h} D u\right|^{2} \mathrm{~d} x \mathrm{~d} t
$$

From (2.2) we find that $|\tilde{\mathcal{A}}(h)| \leqslant L$ so that

$$
\begin{aligned}
2 \int_{t_{0}} \int_{\Omega} \zeta^{2} \eta|\mathcal{A}(h)|\left|\nabla \eta \| \tau_{h} u\right| \mathrm{d} x \mathrm{~d} t & \leqslant 2 L \int_{t_{0}}^{t_{1}} \int_{\Omega} \zeta^{2} \eta\left|\nabla \eta\left\|\tau_{h} D u\right\| \tau_{h} u\right| \mathrm{d} x \mathrm{~d} t \\
& \leqslant \varepsilon \int_{t_{0}}^{t_{1}} \int_{\Omega} \zeta^{2} \eta^{2}\left|\tau_{h} D u\right|^{2} \mathrm{~d} x \mathrm{~d} t+\frac{L^{2}}{\varepsilon} \int_{t_{0}}^{t_{1}} \int_{\mathrm{st} \eta} \zeta^{2}|\nabla \eta|^{2}\left|\tau_{h} u\right|^{2} \mathrm{~d} x \mathrm{~d} t \\
& \leqslant \varepsilon \int_{t_{0}}^{t_{1}} \int_{\Omega} \zeta^{2} \eta^{2}\left|\tau_{h} D u\right|^{2} \mathrm{~d} x \mathrm{~d} t+\frac{L^{2}}{\varepsilon}\|\nabla \eta\|_{L^{\infty}}^{2} \int_{t_{0}}^{t_{1}} \int_{\mathrm{spt} \eta} \zeta^{2}\left|\tau_{h} u\right|^{2} \mathrm{~d} x \mathrm{~d} t \\
& \leqslant \varepsilon \int_{t_{0}}^{t_{1}} \int_{\Omega} \zeta^{2} \eta^{2}\left|\tau_{h} D u\right|^{2} \mathrm{~d} x \mathrm{~d} t+c_{\varepsilon}\|\nabla \eta\|_{L^{\infty}}^{2}|h| \int_{Q_{T}}(1+|D u|)^{2} \mathrm{~d} z,
\end{aligned}
$$

where $c_{\varepsilon}=c\left(\frac{1}{\varepsilon}, L, \operatorname{dist}(\operatorname{spt} \eta, \partial \Omega)\right)$. In the last line we used Corollary 9.2.

Estimates for integrals involving $\mathcal{C}(h)$. Using (2.9), we have $|\mathcal{C}(h)| \leqslant L|h|^{\beta / 2}(1+|D u|)$ and so

$$
\int_{t_{0}}^{t_{1}} \int_{\Omega} \zeta^{2} \eta^{2}|\mathcal{C}(h)|\left|D\left(\tau_{h} u\right)\right| \mathrm{d} x \mathrm{~d} t \leqslant L|h|^{\beta / 2} \int_{t_{0}}^{t_{1}} \int_{\Omega} \zeta^{2} \eta^{2}(1+|D u|)\left|\tau_{h} D u\right| \mathrm{d} x \mathrm{~d} t
$$




$$
\begin{aligned}
& \leqslant \varepsilon \int_{t_{0}}^{t_{1}} \int_{\Omega} \zeta^{2} \eta^{2}\left|\tau_{h} D u\right|^{2} \mathrm{~d} x \mathrm{~d} t+\frac{L^{2}}{\varepsilon}|h|^{\beta} \int_{t_{0}}^{t_{1}} \int_{\Omega} \zeta^{2} \eta^{2}(1+|D u|)^{2} \mathrm{~d} x \mathrm{~d} t \\
& \leqslant \varepsilon \int_{t_{0}}^{t_{1}} \int_{\Omega} \zeta^{2} \eta^{2}\left|\tau_{h} D u\right|^{2} \mathrm{~d} x \mathrm{~d} t+c_{\varepsilon}|h|^{\beta} \int_{Q_{T}}(1+|D u|)^{2} \mathrm{~d} z,
\end{aligned}
$$

where $c_{\varepsilon}=c\left(\frac{1}{\varepsilon}, L\right)$. Using again Corollary 9.2 we gain

$$
\begin{aligned}
2 \int_{t_{0}} \int_{\Omega} \zeta^{2} \eta|\mathcal{C}(h)||\nabla \eta|\left|\tau_{h} u\right| \mathrm{d} x \mathrm{~d} t & \leqslant 2\left(\iint_{t_{0}}^{t_{1}} \zeta^{2}|\mathcal{C}(h)|^{2} \mathrm{~d} x \mathrm{~d} t\right)^{1 / 2}\left(\int_{t_{0}}^{t_{1}} \int_{\Omega} \zeta^{2} \eta^{2}\left|\tau_{h} u\right|^{2} \mathrm{~d} x \mathrm{~d} t\right)^{1 / 2} \\
& \leqslant L|h|^{\beta / 2}\left(\int_{Q_{T}}(1+|D u|)^{2} \mathrm{~d} z\right)^{1 / 2}\left(\int_{t_{0}}^{t_{1}} \int_{\text {spt } \eta}\left|\tau_{h} u\right|^{2} \mathrm{~d} x \mathrm{~d} t\right)^{1 / 2} \\
& \leqslant c|h|^{(1+\beta) / 2} \int_{Q_{T}}(1+|D u|)^{2} \mathrm{~d} z
\end{aligned}
$$

where now $c=c(L, \operatorname{dist}(\operatorname{spt} \eta, \partial \Omega))$.

Estimates for integrals involving $\mathcal{B}(h)$. Here we shall use the fact that there exists $\sigma \equiv \sigma(L / \lambda)>0$ such that

$$
|D u| \in L_{\text {loc }}^{2(1+\sigma)}\left(Q_{T}\right) .
$$

More precisely, see also Theorem 10.1 below: For every open subset $\mathcal{O} \Subset Q_{T}$ there exists a constant $c \equiv$ $c\left(\operatorname{dist}\left(\mathcal{O}, \partial Q_{T}\right)\right)$ such that

$$
\int_{\mathcal{O}}|D u|^{2(1+\sigma)} \mathrm{d} z \leqslant c\left(\int_{Q_{T}}\left(|D u|^{2}+1\right) \mathrm{d} z\right)^{1+\sigma} .
$$

This is a Gehring's type higher integrability result $[17,35,24]$ and we note that in general

$$
\lim _{L / \lambda \uparrow \infty} \sigma=0 .
$$

Using that $|\mathcal{B}(h)| \leqslant L \omega\left(\left|\tau_{h} u\right|\right)(1+|D u|)$, Young's inequality once again and keeping into account that $\omega \leqslant 1$ we have

$$
\begin{aligned}
\int_{t_{0}}^{t_{1}} \int_{\Omega} \zeta^{2} \eta^{2}|\mathcal{B}(h)|\left|\tau_{h} D u\right| \mathrm{d} x \mathrm{~d} t & \leqslant \varepsilon \int_{t_{0}}^{t_{1}} \int_{\Omega} \zeta^{2} \eta^{2}\left|\tau_{h} D u\right|^{2} \mathrm{~d} x \mathrm{~d} t+\frac{1}{\varepsilon} \int_{t_{0}}^{t_{1}} \int_{\Omega} \zeta^{2} \eta^{2}|\mathcal{B}(h)|^{2} \mathrm{~d} x \mathrm{~d} t \\
& \leqslant \varepsilon \int_{t_{0}}^{t_{1}} \int_{\Omega} \zeta^{2} \eta^{2}\left|\tau_{h} D u\right|^{2} \mathrm{~d} x \mathrm{~d} t+\frac{L^{2}}{\varepsilon} \int_{t_{0}}^{t_{1}} \int_{\text {spt } \eta} \omega\left(\left|\tau_{h} u\right|\right)(1+|D u|)^{2} \mathrm{~d} x \mathrm{~d} t .
\end{aligned}
$$

It remains to estimate the second integral appearing on the right-hand side. Using Hölder's inequality, the higher integrability of $D u$ twice with (9.10), Jensen's inequality and once again the estimate for the fractional time derivative of $u$ we obtain 


$$
\begin{aligned}
& \int_{t_{0} \text { spt } \eta}^{t_{1}} \omega\left(\left|\tau_{h} u\right|\right)(1+|D u|)^{2} \mathrm{~d} x \mathrm{~d} t \\
& \quad \leqslant|\operatorname{spt} \eta|\left(t_{1}-t_{0}\right)\left(f_{\operatorname{spt} \eta \times\left(t_{0}, t_{1}\right)} \omega\left(\left|\tau_{h} u\right|\right)^{\frac{1+\sigma}{\sigma}} \mathrm{d} z\right)^{\frac{\sigma}{1+\sigma}}\left(f_{\operatorname{spt} \eta \times\left(t_{0}, t_{1}\right)}(1+|D u|)^{2(1+\sigma)} \mathrm{d} z\right)^{\frac{1}{1+\sigma}} \\
& \quad \leqslant|\operatorname{spt} \eta|\left(t_{1}-t_{0}\right) \omega\left(f_{\operatorname{spt} \eta \times\left(t_{0}, t_{1}\right)}\left|\tau_{h} u\right| \mathrm{d} z\right)^{\frac{\sigma}{1+\sigma}}\left(f_{\operatorname{spt} \eta \times\left(t_{0}, t_{1}\right)}(1+|D u|)^{2(1+\sigma)} \mathrm{d} z\right)^{\frac{1}{1+\sigma}} \\
& \quad \leqslant|\operatorname{spt} \eta|\left(t_{1}-t_{0}\right)\left(\int_{\operatorname{spt} \eta \times\left(t_{0}, t_{1}\right)}\left|\tau_{h} u\right|^{2} \mathrm{~d} z\right)^{\frac{\beta \sigma}{2(1+\sigma)}}\left(f_{\operatorname{spt} \eta \times\left(t_{0}, t_{1}\right)}(1+|D u|)^{2(1+\sigma)} \mathrm{d} z\right)^{\frac{1}{1+\sigma}} \\
& \leqslant c\left(\int_{Q_{T}}(1+|D u|)^{2} \mathrm{~d} z\right)^{\frac{\beta \sigma}{2(1+\sigma)}}\left(\int_{Q_{T}}(1+|D u|)^{2} \mathrm{~d} z\right)_{|h|^{\frac{\beta \sigma}{2(1+\sigma)}}} \\
& \leqslant c\left(\beta, \sigma,\left|Q_{T}\right|, \operatorname{dist}(\operatorname{spt} \eta, \partial \Omega),\|D u\|_{L^{2}\left(Q_{T}\right)}\right)|h|^{\frac{\beta \sigma}{2(1+\sigma)}} .
\end{aligned}
$$

In the second last line we also used (9.10). For the remaining term we have

$$
\begin{aligned}
& \int_{t_{0}}^{t_{1}} \int_{\Omega} \zeta^{2} \eta|\mathcal{B}(h)||\nabla \eta|\left|\tau_{h} u\right| \mathrm{d} x \mathrm{~d} t \\
& \quad \leqslant\|\nabla \eta\|_{L^{\infty}}\left(\int_{t_{0} \mathrm{spt} \eta}^{t_{1}} \int^{2} \eta^{2}|\mathcal{B}(h)|^{2} \mathrm{~d} x \mathrm{~d} t\right)^{1 / 2}\left(\int_{t_{0}}^{t_{1}} \int_{\mathrm{spt} \eta}\left|\tau_{h} u\right|^{2} \mathrm{~d} x \mathrm{~d} t\right)^{1 / 2} .
\end{aligned}
$$

Now, the first integral appearing in the right-hand side of the previous estimate can be treated as the third integral in (9.12), while the second integral can be estimated via the estimate of the fractional time derivative for $u$ from Corollary 9.2. We finally arrive at

$$
\int_{t_{0}}^{t_{1}} \int_{\Omega} \zeta^{2} \eta|\mathcal{B}(h)||\nabla \eta|\left|\tau_{h} u\right| \mathrm{d} x \mathrm{~d} t \leqslant c|h|^{\frac{1}{2}+\frac{\beta \sigma}{4(1+\sigma)}} \leqslant c|h|^{\frac{\beta \sigma}{2(1+\sigma)}},
$$

where $c=c\left(L, \beta, \sigma,\left|Q_{T}\right|, \operatorname{dist}(\operatorname{spt} \eta, \partial \Omega),\|D u\|_{L^{2}\left(Q_{T}\right)},\|\nabla \eta\|_{L^{\infty}}\right)$.

We now turn our attention to the remaining term from the left-hand side of (9.8). Recalling that $0 \leqslant \zeta^{\prime} \leqslant \frac{4}{T+t_{0}}$ this last term is estimated again via Corollary 9.2. We obtain

$$
\int_{t_{0}}^{\tilde{t}} \int_{\mathrm{spt} \eta} \zeta \zeta^{\prime} \eta^{2}\left|\tau_{h} u\right|^{2} \mathrm{~d} x \mathrm{~d} t \leqslant \frac{4}{T+t_{0}} \int_{t_{0}}^{t_{1}} \int_{\Omega}\left|\tau_{h} u\right|^{2} \mathrm{~d} x \mathrm{~d} t \leqslant c|h| \int_{Q_{T}}(1+|D u|)^{2} \mathrm{~d} z,
$$

where $c=c\left(L, \operatorname{dist}(\operatorname{spt} \eta, \partial \Omega), t_{0}\right)$.

Connecting the previous estimates and taking $\varepsilon$ in terms of $\lambda$ suitably small we finally arrive at

$$
\int_{t_{0}}^{t_{1}} \int_{\Omega} \zeta^{2} \eta^{2}\left|\tau_{h} D u\right|^{2} \mathrm{~d} x \mathrm{~d} t \leqslant c_{1}\left(|h|+|h|^{\beta}+|h|^{\frac{1+\beta}{2}}\right)+c_{2}|h|^{\frac{\beta \sigma}{2(1+\sigma)}} \leqslant c_{1}|h|^{\beta}+c_{2}|h|^{\frac{\beta \sigma}{2(1+\sigma)}},
$$


where

$$
c_{1}=c\left(\lambda, L,\left|Q_{T}\right|, \operatorname{dist}(\operatorname{spt} \eta, \partial \Omega), t_{0},\|\nabla \eta\|_{\left.L^{\infty},\|D u\|_{L^{2}\left(Q_{T}\right)}\right)}\right.
$$

and

$$
c_{2}=c\left(\lambda, L, \beta, \sigma,\left|Q_{T}\right|, \operatorname{dist}(\operatorname{spt} \eta, \partial \Omega),\|\nabla \eta\|_{\left.L^{\infty},\|D u\|_{L^{2}\left(Q_{T}\right)}\right)} .\right.
$$

Remark 9.3. In the case of systems of the type (1.4), the above estimate holds with $c_{2}=0$. Indeed the last term in (9.13) is due to the presence of $\mathcal{B}(h)$.

\section{Letting}

$$
\delta:=\frac{\beta \sigma}{1+\sigma}
$$

and using the fact that $\widetilde{\Omega}, \eta, \zeta, t_{0}, t_{1}$ considered above were arbitrary, we can therefore conclude, by Proposition 3.4

Lemma 9.4. Let $u \in L^{2}\left(-T, 0 ; W^{1,2}\left(\Omega, \mathbb{R}^{N}\right)\right)$ be a weak solution of the nonlinear parabolic system (1.1) under the assumptions (2.1)-(2.3) and (2.9). Then for any $\widetilde{\Omega} \Subset \Omega,\left(t_{0}, t_{1}\right) \Subset(-T, 0)$ we have in the special case of coefficients $A \equiv A(x, t, D u)$ that

$$
\iint_{\widetilde{\Omega}} \int_{t_{0}}^{t_{1}} \frac{|D u(x, t)-D u(x, s)|^{2}}{|t-s|^{1+2 \gamma}} \mathrm{d} t \mathrm{~d} s \mathrm{~d} x<\infty, \quad \forall \gamma<\frac{\beta}{2},
$$

while in the general case $A \equiv A(x, t, u, D u)$ we have that

$$
\iint_{\widetilde{\Omega}} \int_{t_{0}}^{t_{1}} \int_{t_{0}}^{t_{1}} \frac{|D u(x, t)-D u(x, s)|^{2}}{|t-s|^{1+2 \gamma}} \mathrm{d} t \mathrm{~d} s \mathrm{~d} x<\infty, \quad \forall \gamma<\frac{\delta}{4} .
$$

Now we pass to the fractional space derivatives of $D u$. In many points, the following estimates will be similar to the ones for fractional time derivatives; therefore many arguments will only be sketched. Denoting by $\left\{e_{s}\right\}_{1 \leqslant s \leqslant n}$ the standard basis for $\mathbb{R}^{n}$ we shall set, as for the time derivative case

$$
\tau_{h}^{s} u \equiv\left(\tau_{h}^{s} u\right)(x, t):=u\left(x+h e_{s}, t\right)-u(x, t)
$$

whenever $\left(x+h e_{s}, t\right),(x, t) \in Q_{T}$. Let us observe that since the solution $u$ is assumed to be weakly differentiable with respect to space i.e. $u \in L^{2}\left(-T, 0 ; W^{1,2}\left(\Omega, \mathbb{R}^{N}\right)\right)$, then the space analogue of estimate (9.2) comes for free:

$$
\int_{-T \widetilde{\Omega}}^{0}\left|u\left(x+h e_{s}, t\right)-u(x, t)\right|^{2} \mathrm{~d} x \mathrm{~d} t \leqslant c|h|^{2} \int_{Q_{T}}\left(1+|D u|^{2}\right) \mathrm{d} z,
$$

whenever $\widetilde{\Omega} \Subset \Omega$ and $|h| \leqslant \frac{1}{2} \min \{\operatorname{dist}(\widetilde{\Omega}, \partial \Omega), 1\} ; c \equiv c(n)$. The previous estimate will be used in the following to replace (9.2). Once again we start from the formulation via Steklov averages in (9.5) and we select $\widetilde{\Omega}$ and $h$ as specified above. Replacing $\varphi$ by $\tau_{-h}^{s} \varphi$, where $\varphi \in W_{0}^{1,2}\left(\widetilde{\Omega}, \mathbb{R}^{N}\right)$, and using "integration by parts" we have

$$
\int_{\widetilde{\Omega}}\left(\partial_{t}\left(\tau_{h}^{s} u_{\lambda}\right) \varphi+\tau_{h}^{s}[A(x, t, u, D u)]_{\lambda} D \varphi\right) \mathrm{d} z=0 .
$$

In the previous relation we use the test function $\varphi(x, t):=\zeta^{2}(t) \eta^{2}(x)\left(\tau_{h}^{s} u_{\lambda}\right)(x, t)$ where $\zeta(t)$ is from (9.4) and $\eta \in C_{0}^{\infty}(\widetilde{\Omega})$ is a cut-off in space. We repeat the manipulations done for the time derivative case and obtain the following analogue of (9.7) 


$$
\begin{aligned}
& \frac{1}{2} \int_{\widetilde{\Omega}} \zeta^{2}\left(t_{1}\right) \eta^{2}\left|\tau_{h}^{s} u\right|^{2}\left(\cdot, t_{1}\right) \mathrm{d} x-\int_{-T} \int_{\widetilde{\Omega}}^{t_{1}} \zeta \zeta^{\prime} \eta^{2}\left|\tau_{h}^{s} u\right|^{2} \mathrm{~d} x \mathrm{~d} t \\
& \quad+\int_{t_{0}} \int_{\widetilde{\Omega}}^{t_{1}} \zeta^{2} \tau_{h}^{s}[A(x, t, u, D u)]\left[2 \eta \nabla \eta \otimes\left(\tau_{h}^{s} u\right)+\eta^{2} D\left(\tau_{h}^{s} u\right)\right] \mathrm{d} x \mathrm{~d} t=0 .
\end{aligned}
$$

Now we decompose $\tau_{h}^{s}(A)$ as follows:

$$
\begin{aligned}
\tau_{h}^{s}[ & A(\cdot, \cdot, u(\cdot, \cdot), D u(\cdot, \cdot))](x, t) \\
= & A\left(x+h e_{s}, t, u\left(x+h e_{s}, t\right), D u\left(x+h e_{s}, t\right)\right)-A(x, t, u(x, t), D u(x, t)) \\
= & A\left(x+h e_{s}, t, u\left(x+h e_{s}, t\right), D u\left(x+h e_{s}, t\right)\right)-A\left(x+h e_{s}, t, u\left(x+h e_{s}, t\right), D u(x, t)\right) \\
& +A\left(x+h e_{s}, t, u\left(x+h e_{s}, t\right), D u(x, t)\right)-A\left(x+h e_{s}, t, u(x, t), D u(x, t)\right) \\
& +A\left(x+h e_{s}, t, u(x, t), D u(x, t)\right)-A(x, t, u(x, t), D u(x, t)) \\
= & : \mathcal{A}(h)+\mathcal{B}(h)+\mathcal{C}(h) .
\end{aligned}
$$

As for the time derivative case we discard the first integral in (9.18) and estimate the remaining terms as in the time derivatives case, with suitable variants. By ellipticity (2.3) we infer

$$
\int_{t_{0}}^{t_{1}} \int_{\widetilde{\Omega}} \zeta^{2} \eta^{2} \mathcal{A}(h) D\left(\tau_{h}^{s} u\right) \mathrm{d} x \mathrm{~d} t \geqslant \lambda \int_{t_{0}}^{t_{1}} \int_{\widetilde{\Omega}} \zeta^{2} \eta^{2}\left|\tau_{h}^{s} D u\right|^{2} \mathrm{~d} x \mathrm{~d} t
$$

while using (9.17) and Young's inequality, for any $\varepsilon \in(0,1)$, we see that

$$
\left.\int_{t_{0}}^{t_{1}} \int_{\widetilde{\Omega}} \zeta^{2} \eta|\mathcal{A}(h)|\left|\nabla \eta\left\|\left.\tau_{h}^{s} u\left|\mathrm{~d} x \mathrm{~d} t \leqslant \varepsilon \int_{t_{0}}^{t_{1}} \int_{\widetilde{\Omega}} \zeta^{2} \eta^{2}\right| \tau_{h}^{s} D u\right|^{2} \mathrm{~d} x \mathrm{~d} t+c_{\varepsilon}(L)\right\| \nabla \eta \|_{L^{\infty}}^{2}\right| h\right|^{2} \int_{Q_{T}}(1+|D u|)^{2} \mathrm{~d} z .
$$

Again, using (2.9)

$$
\int_{t_{0}}^{t_{1}} \int_{\widetilde{\Omega}} \zeta^{2} \eta^{2}|\mathcal{C}(h)|\left|\tau_{h}^{s} D u\right| \mathrm{d} x \mathrm{~d} t \leqslant \varepsilon \int_{t_{0}}^{t_{1}} \int_{\widetilde{\Omega}} \zeta^{2} \eta^{2}\left|\tau_{h}^{s} D u\right|^{2} \mathrm{~d} x \mathrm{~d} t+c_{\varepsilon}(L)|h|^{2 \beta} \int_{Q_{T}}(1+|D u|)^{2} \mathrm{~d} x \mathrm{~d} t,
$$

and, using (9.17) and Hölder's inequality

$$
\int_{t_{0}}^{t_{1}} \int_{\widetilde{\Omega}} \zeta^{2} \eta^{2}|\mathcal{C}(h)||D \eta|\left|\tau_{h}^{s} u\right| \mathrm{d} x \mathrm{~d} t \leqslant c\left(n, L,\|D \eta\|_{L^{\infty}}\right)|h|^{1+\beta} \int_{Q_{T}}(1+|D u|)^{2} \mathrm{~d} x \mathrm{~d} t .
$$

Concerning the estimates for the integrals involving $\mathcal{B}(h)$ we have, as in the case of time derivatives

$$
\begin{aligned}
& \int_{t_{0}}^{t_{1}} \int_{\text {spt } \eta} \omega\left(\left|\tau_{h}^{S} u\right|\right)(1+|D u|)^{2} \mathrm{~d} x \mathrm{~d} t \leqslant c\left(\int_{Q_{T}}(1+|D u|)^{2} \mathrm{~d} x \mathrm{~d} t\right)^{\frac{\beta \sigma}{2(1+\sigma)}}\left(\int_{Q_{T}}(1+|D u|)^{2} \mathrm{~d} x \mathrm{~d} t\right)|h|^{\frac{\beta \sigma}{1+\sigma}} \\
& \leqslant c_{3}\left(\beta, \sigma,\left|Q_{T}\right|, \operatorname{dist}(\operatorname{spt} \eta, \partial \Omega),\|\nabla \eta\|_{L^{\infty}},\|D u\|_{L^{2}\left(Q_{T}\right)}\right)|h|^{\frac{\beta \sigma}{1+\sigma}} .
\end{aligned}
$$


Therefore, with $\varepsilon \in(0,1)$ we have

$$
\int_{t_{0}}^{t_{1}} \int_{\widetilde{\Omega}} \zeta^{2} \eta^{2}|\mathcal{B}(h)|\left|D \tau_{h}^{s} u\right| \mathrm{d} x \mathrm{~d} t \leqslant \varepsilon \int_{t_{0}}^{t_{1}} \int_{\widetilde{\Omega}} \zeta^{2} \eta^{2}\left|\tau_{h}^{s} D u\right|^{2} \mathrm{~d} x \mathrm{~d} t+c_{\varepsilon} c_{3}|h|^{\frac{\beta \sigma}{1+\sigma}} .
$$

Again we have

$$
\begin{aligned}
& \int_{t_{0}}^{t_{1}} \int_{\widetilde{\Omega}} \zeta^{2} \eta^{2}|\mathcal{B}(h)||D \eta|\left|\tau_{h}^{s} u\right| \mathrm{d} x \mathrm{~d} t \\
& \quad \leqslant c_{4}\left(L, \beta, \sigma,\left|Q_{T}\right| \operatorname{dist}(\operatorname{spt} \eta, \partial \Omega),\|\nabla \eta\|_{\left.L^{\infty},\|D u\|_{L^{2}\left(Q_{T}\right)}\right)|h|^{1+\frac{\beta \sigma}{2(1+\sigma)}},}\right.
\end{aligned}
$$

and, by (9.17)

$$
\int_{t_{0}}^{t_{1}} \int_{\widetilde{\Omega}} \zeta \zeta^{\prime} \eta^{2}\left|\tau_{h}^{s} u\right|^{2} \mathrm{~d} x \mathrm{~d} t \leqslant c\left(t_{0}\right)|h|^{2} \int_{Q_{T}}(1+|D u|)^{2} \mathrm{~d} x \mathrm{~d} t .
$$

Connecting all the previous estimates we finally have

$$
\int_{t_{0}}^{t_{1}} \int_{\widetilde{\Omega}} \zeta^{2} \eta^{2}\left|\tau_{h}^{s} D u\right|^{2} \mathrm{~d} x \mathrm{~d} t \leqslant \tilde{c}_{1}\left(|h|^{2}+|h|^{2 \beta}+|h|^{1+\beta}\right)+\tilde{c}_{2}|h|^{\frac{\beta \sigma}{1+\sigma}} \leqslant \tilde{c}_{1}|h|^{2 \beta}+\tilde{c}_{2}|h|^{\frac{\beta \sigma}{1+\sigma}},
$$

where the constants $\tilde{c}_{1}$ and $\tilde{c}_{2}$ enjoy the same dependencies as the constants $c_{1}$ and $c_{2}$ in (9.14) and (9.15), respectively. Arguing as for Lemma 9.4, the last estimate and Proposition 3.4 allow us to conclude with

Lemma 9.5. Let $u \in L^{2}\left(-T, 0 ; W^{1,2}\left(\Omega, \mathbb{R}^{N}\right)\right)$ be a weak solution of the nonlinear parabolic system (1.1) under the assumptions (2.1)-(2.3) and (2.9). Then for any $\widetilde{\Omega} \Subset \Omega,\left(t_{0}, t_{1}\right) \Subset(-T, 0)$ we have in the special case of coefficients $A \equiv A(x, t, D u)$ that

$$
\iint_{t_{0}} \int_{\widetilde{\Omega}} \int_{\widetilde{\Omega}} \frac{|D u(x, t)-D u(y, t)|^{2}}{|x-y|^{n+2 \gamma}} \mathrm{d} x \mathrm{~d} y \mathrm{~d} t<\infty, \quad \forall \gamma<\beta,
$$

while in the general case $A \equiv A(x, t, u, D u)$ we have that

$$
\iint_{t_{0}} \int_{\widetilde{\Omega}} \int_{\widetilde{\Omega}} \frac{|D u(x, t)-D u(y, t)|^{2}}{|x-y|^{n+2 \gamma}} \mathrm{d} x \mathrm{~d} y \mathrm{~d} t<\infty, \quad \forall \gamma<\frac{\delta}{2} .
$$

Proof of Theorem 1.3. The proof is a consequence of Lemma 9.4, Lemma 9.5 and Proposition 3.3. Indeed we have that

$$
D u \in W_{\mathrm{loc}}^{\gamma, \frac{\gamma}{2} ; 2}\left(Q_{T}, \mathbb{R}^{n N}\right), \quad \forall \gamma<\frac{\delta}{2} .
$$

On the other hand, Corollary 9.2, (9.17) and Proposition 3.4 give

$$
u \in W_{\mathrm{loc}}^{\gamma, \frac{\gamma}{2} ; 2}\left(Q_{T}, \mathbb{R}^{N}\right), \quad \forall \gamma<1,
$$

actually a better result holds, see Proposition 10.5 below. Therefore, applying Proposition 3.3 simultaneously to both $D u$ and $u$ we conclude that

$$
\operatorname{dim}_{\mathcal{P}}\left(\Sigma_{1}\right) \leqslant n+2-\delta, \quad \operatorname{dim}_{\mathcal{P}}\left(\Sigma_{2}\right) \leqslant n+2-\delta .
$$


The dependence of the $\delta$ described in (1.3) comes directly from the definition of $\delta$ in (9.16) and the property in (9.11). Let us observe that the same reasoning applied to the case of systems of the type (1.4) would give

$$
\operatorname{dim}_{\mathcal{P}}\left(\Sigma_{1}\right) \leqslant n+2-2 \beta, \quad \operatorname{dim}_{\mathcal{P}}\left(\Sigma_{2}\right) \leqslant n+2-2 \beta
$$

the aim of the next section is to improve the last inequalities coming to the full proof of Theorem 1.4.

Remark 9.6. As mentioned in Section 1, the constant $\delta$ appearing in Theorem 1.3 and (9.16), can be quantified. Indeed it directly depends on $\sigma$, defined in (9.9) and coming from the proof of Gehring's lemma in the parabolic case $[24,17]$. The exponent $\sigma$ essentially depends on the parameter $L / \lambda$ as in $(9.11)$ and can be in turn quantified. This can be done, for instance, following the lines of the proofs in [24] and seeing that in turn the exponents involved in Gehring's lemma can be quantified as well; for such issues we refer for instance to [3,43]. The same argumentation applies to Theorem 1.4.

\section{Proof of Theorem 1.4}

In this section we specialize to systems of the type in (1.4), under the assumptions already considered in Section 2, when obviously restated for such a case. Once again we shall use higher integrability properties of solutions, but this time we shall also need the fact that gradients satisfy certain Reverse-Hölder inequalities; this was not strictly needed before. To this aim we recall the following result, which in a more complete form can be found in [35], see also [17]:

Theorem 10.1. Let $v \in L^{2}\left(-T, 0 ; W^{1,2}\left(\Omega, \mathbb{R}^{N}\right)\right)$ be a weak solution to the system

$$
v_{t}-\operatorname{div} A(x, t, v, D v)=\operatorname{div} C(x, t),
$$

under the assumptions (2.1)-(2.3), where $C \in L_{\text {loc }}^{2(1+\sigma)}\left(Q_{T}, \mathbb{R}^{n N}\right)$ and $\sigma \in(0,1)$. Then there exists $\sigma_{0} \equiv$ $\sigma_{0}(n, L / \lambda) \in(0, \sigma)$ such that $|D u| \in L_{\text {loc }}^{2\left(1+\sigma_{0}\right)}\left(Q_{T}\right)$. Moreover there exists $c \equiv c(n, L / \lambda)$ such that for every $Q_{2 \varrho} \Subset Q_{T}$ the following Reverse-Hölder inequality holds:

$$
\left(f_{Q_{\varrho}}|D u|^{2\left(1+\sigma_{0}\right)} \mathrm{d} z\right)^{1 /\left(1+\sigma_{0}\right)} \leqslant c f_{Q_{2 \varrho}}(1+|D u|)^{2} \mathrm{~d} z+c\left(f_{Q_{2 \varrho}}|C(z)|^{2(1+\sigma)} \mathrm{d} z\right)^{1 /(1+\sigma)} .
$$

Remark 10.2. Inequalities similar to (10.1) can also be obtained for systems with $p$-growth following the techniques in $[24,32]$. The proof is of course much more involved than the one in the case $p=2$. We emphasize here that, the possibility to have a pure Reverse-Hölder inequality as in (10.1), that is an inequality which is homogeneous, is restricted to the case $p=2$.

We start deriving other fractional estimates, that in turn build up on (9.13)-(9.19). In the weak formulation

$$
\int_{Q_{T}}\left(u \partial_{t} \varphi-A(x, t, D u) D \varphi\right) \mathrm{d} z=0,
$$

valid for any $\varphi \in C_{0}^{\infty}\left(Q_{T}, \mathbb{R}^{n}\right)$, we switch $\varphi$ to $\tau_{-h}^{s} \varphi$, where, as usual $s \in\{1, \ldots, n\}, \widetilde{Q}:=\widetilde{\Omega} \times(-T+d,-d)$, $d \in(0, T / 4), \widetilde{\Omega} \Subset \Omega$ is an open subset, $\varphi \in C_{0}^{\infty}\left(\widetilde{Q}, \mathbb{R}^{N}\right)$ and $0<|h|<\frac{1}{2} \min \{\operatorname{dist}(\widetilde{\Omega}, \partial \Omega), 1\}$. "Integration by parts" yields

$$
\int_{Q_{T}}\left(\tau_{h}^{s} u \partial_{t} \varphi-\tau_{h}^{s}[A(x, t, D u)] D \varphi\right) \mathrm{d} z=0
$$


Similarly to the estimation of the fractional space derivatives of $D u$ in the previous section we write

$$
\begin{aligned}
\tau_{h}^{s}[A(\cdot, \cdot, D u(\cdot, \cdot))](x, t)= & A\left(x+h e_{s}, t, D u\left(x+h e_{s}, t\right)\right)-A(x, t, D u(x, t)) \\
= & A\left(x+h e_{s}, t, D u\left(x+h e_{s}, t\right)\right)-A\left(x+h e_{s}, t, D u(x, t)\right) \\
& +A\left(x+h e_{s}, t, D u(x, t)\right)-A(x, t, D u(x, t)) \\
= & : \mathcal{A}(h)+\mathcal{C}(h) .
\end{aligned}
$$

In turn

$$
\mathcal{A}(h)(x, t)=\int_{0}^{1} \frac{\partial A}{\partial p}\left(x+h e_{s}, t, D u(x, t)+s \tau_{h}^{s}(D u)(x, t)\right) \mathrm{d} s \tau_{h}^{s}(D u)(x, t)=: \tilde{\mathcal{A}}(h) \tau_{h}^{s}(D u)(x, t) .
$$

With these abbreviations (10.3) can be re-written as

$$
\int_{\widetilde{Q}}\left(\tau_{h}^{s} u \partial_{t} \varphi-\tilde{\mathcal{A}}(h) \tau_{h}^{s} D u D \varphi\right) \mathrm{d} z=-\int_{\widetilde{Q}} \mathcal{C}(h) D \varphi \mathrm{d} z,
$$

for every $\varphi \in C_{0}^{\infty}\left(\widetilde{Q}, \mathbb{R}^{N}\right)$. Therefore, if we let

$$
v_{h}:=\frac{\tau_{h}^{s} u}{|h|^{\beta}}, \quad \tilde{\mathcal{C}}(h):=-\frac{\mathcal{C}(h)}{|h|^{\beta}}
$$

and divide (10.3) by $|h|^{\beta}$, we obtain that $v_{h}$ solves a linear parabolic system with measurable and bounded coefficients i.e.

$$
\int_{\widetilde{Q}}\left(v_{h} \partial_{t} \varphi-\tilde{\mathcal{A}}(h) D v_{h} D \varphi\right) \mathrm{d} z=\int_{\widetilde{Q}} \tilde{\mathcal{C}}(h) D \varphi \mathrm{d} z
$$

for any $\varphi \in C_{0}^{\infty}\left(\widetilde{Q}, \mathbb{R}^{N}\right)$. Indeed, using the (2.2) and (2.3) we infer that $\mathcal{A}(h)$ satisfies the uniform (in $h$ ) bounds

$$
\lambda|p|^{2} \leqslant \mathcal{A}(h) p \cdot p, \quad|\mathcal{A}(h)| \leqslant L, \quad \forall p \in \mathbb{R}^{n N} .
$$

Moreover, using the fact that $|\mathcal{C}(h)| \leqslant L|h|^{\beta}(1+|D u|)$ and that $|D u| \in L_{\text {loc }}^{2(1+\sigma)}\left(Q_{T}\right)$ we see that

$$
|\tilde{\mathcal{C}}(h)| \in L_{\text {loc }}^{2(1+\sigma)}\left(Q_{T}\right) .
$$

Therefore we are able to apply to the results of Theorem 10.1 to the linear parabolic system (10.4) to infer the existence of $0<\sigma_{0} \equiv \sigma_{0}(n, L / \lambda)<\sigma$, independent of $h$, such that, after a routine estimation

$$
\begin{aligned}
\left(f_{Q_{\varrho} / 2}\left|D v_{h}\right|^{2\left(1+\sigma_{0}\right)} \mathrm{d} z\right)^{1 /\left(1+\sigma_{0}\right)} & \leqslant c f_{Q_{\varrho}}\left|D v_{h}\right|^{2} \mathrm{~d} z+c\left(f_{Q_{\varrho}}(1+|\tilde{\mathcal{C}}(h)|)^{2(1+\sigma)} \mathrm{d} z\right)^{1 /(1+\sigma)} \\
& \leqslant c f_{Q_{\varrho}}\left|D v_{h}\right|^{2} \mathrm{~d} z+c\left(f_{\widetilde{Q}_{\varrho}}(1+|D u|)^{2(1+\sigma)} \mathrm{d} z\right)^{1 /(1+\sigma)}
\end{aligned}
$$

whenever $Q_{2 \varrho} \Subset \widetilde{Q}$; in the last line we used the fact that $|\tilde{\mathcal{C}}(h)| \leqslant L(1+|D u|)$, which is a direct consequence of (2.9). We explicitly observe that all the constants involved in the previous estimates are independent of $h$. Using (9.19) (with $\tilde{c}_{2}=0$, see Remark 9.3) to estimate the second integral (with a suitable choice of $\eta$ and $\zeta$ ) we have 


$$
\begin{aligned}
\left(\int_{Q_{\varrho / 2}}\left|D v_{h}\right|^{2\left(1+\sigma_{0}\right)} \mathrm{d} z\right)^{1 /\left(1+\sigma_{0}\right)} & \leqslant c \varrho^{-(n+2)} \tilde{c}_{1}+\left(f_{Q_{\varrho}}(1+|D u|)^{2(1+\sigma)} \mathrm{d} z\right)^{1 /(1+\sigma)} \\
& \leqslant \frac{\tilde{c}_{1}}{\varrho^{n+2}}+\frac{c\left(\operatorname{dist}\left(Q_{\varrho}, Q_{T}\right)\right)}{\varrho^{n+2}} \int_{Q_{T}}(1+|D u|)^{2} \mathrm{~d} z
\end{aligned}
$$

where in the last line we made use of (9.10). The previous estimate and the definition of $v_{h}$ now give

$$
\int_{Q_{2 \varrho}}\left|\tau_{h}^{s} D u\right|^{2\left(1+\sigma_{0}\right)} \mathrm{d} z \leqslant c|h|^{2 \beta\left(1+\sigma_{0}\right)},
$$

where $c$ exhibits the same dependencies as $\tilde{c}_{1}$ and depends also on $\varrho$ and $\operatorname{dist}\left(Q_{2 \varrho}, \widetilde{Q}\right)$. Therefore, keeping into account that the choice of $Q_{2 \varrho} \Subset \widetilde{\Omega}$ was arbitrary, by a standard covering argument we can conclude that, for every open subset $\mathcal{O} \Subset \widetilde{\Omega}$ there exists a constant $c$, essentially depending on $L / \lambda$ and $\operatorname{dist}(\mathcal{O}, \widetilde{Q})$, such that

$$
\int_{\mathcal{O}}\left|\tau_{h}^{s} D u\right|^{2\left(1+\sigma_{0}\right)} \mathrm{d} z \leqslant c|h|^{2 \beta\left(1+\sigma_{0}\right)}, \quad \forall s \in\{1, \ldots, n\} .
$$

In turn, using the fact that $\widetilde{Q}$ was arbitrary, the previous inequality and Proposition 3.4 yield the following improvement of Lemma 9.5:

Lemma 10.3. Let $u \in L^{2}\left(-T, 0 ; W^{1,2}\left(\Omega, \mathbb{R}^{N}\right)\right)$ be a weak solution of the nonlinear parabolic system (1.4) under the assumptions (2.1)-(2.3) and (2.9). Then for any $\widetilde{\Omega} \Subset \Omega,\left(t_{0}, t_{1}\right) \Subset(-T, 0)$ we have that

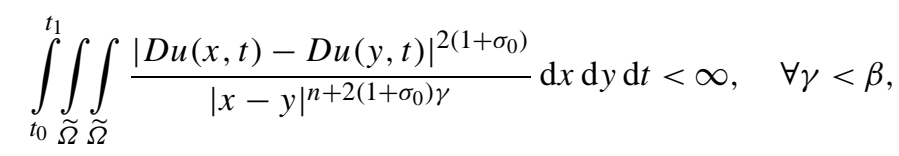

where

$$
\sigma_{0} \equiv \sigma_{0}(L / \lambda)>0 .
$$

Now we are going to derive the time analogue of the last result. Keeping the above notations, substituting $\tau_{-h} \varphi$ in (10.2), where $h$ is as in (9.3) and furthermore satisfies $|h| \leqslant d / 4$, we find

$$
\int_{Q_{T}}\left(\tau_{h} u \partial_{t} \varphi-\tau_{h}[A(x, t, D u)] D \varphi\right) \mathrm{d} z=0 .
$$

Arguing as above we split this time

$$
\begin{aligned}
\tau_{h}[A(\cdot, \cdot, D u(\cdot, \cdot))](x, t)= & A(x, t+h, D u(x, t+h))-A(x, t, D u(x, t)) \\
= & A(x, t+h, D u(x, t+h))-A(x, t+h, D u(x, t)) \\
& +A(x, t+h, D u(x, t))-A(x, t, D u(x, t)) \\
= & : \mathcal{A}(h)+\mathcal{D}(h),
\end{aligned}
$$

and write

$$
\mathcal{A}(h)(x, t)=\int_{0}^{1} \frac{\partial A}{\partial p}\left(x, t+h, D u(x, t)+s \tau_{h}(D u)(x, t)\right) \mathrm{d} s \tau_{h}(D u)(x, t)=: \tilde{\mathcal{A}}(h) \tau_{h}(D u)(x, t) .
$$


Letting now

$$
v_{h}:=\frac{\tau_{h} u}{|h|^{\beta / 2}}, \quad \widetilde{\mathcal{D}}(h):=-\frac{\mathcal{D}(h)}{|h|^{\beta / 2}},
$$

as before, we see that $v_{h}$ is a solution of the linear parabolic system

$$
\int_{\widetilde{Q}}\left(v_{h} \partial_{t} \varphi-\tilde{\mathcal{A}}(h) D v_{h} D \varphi\right) \mathrm{d} z=\int_{\widetilde{Q}} \widetilde{\mathcal{D}}(h) D \varphi \mathrm{d} z
$$

for every $\varphi \in C_{0}^{\infty}\left(\widetilde{Q}, \mathbb{R}^{N}\right)$. Then we note that $|\widetilde{\mathcal{D}}(h)| \leqslant L(1+|D u|)$ so that $|\widetilde{\mathcal{D}}(h)| \in L_{\text {loc }}^{2(1+\sigma)}\left(Q_{T}\right)$. Therefore we may proceed as for the spatial case i.e.: using Theorem 10.1 to derive the time derivative analogue of (10.6), but this time via the use of (9.13) with $c_{2} \equiv 0$, see Remark 9.3. Then, using Proposition 3.4 as above we come up with the following improvement of Lemma 10.3:

Lemma 10.4. Let $u \in L^{2}\left(-T, 0 ; W^{1,2}\left(\Omega, \mathbb{R}^{N}\right)\right)$ be a weak solution of the nonlinear parabolic system (1.4) under the assumptions (2.1)-(2.3) and (2.9). Then for any $\widetilde{\Omega} \Subset \Omega,\left(t_{0}, t_{1}\right) \Subset(-T, 0)$ we have that

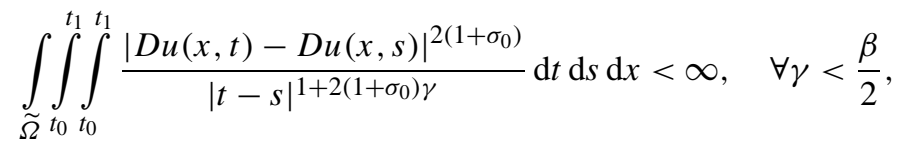

where $\sigma_{0}$ is as in Lemma 10.3 .

Proof of Theorem 1.4. The proof is similar as the one of Theorem 1.3 but this time we shall refer to Theorem 8.2 rather than Theorem 8.1, therefore we have to estimate the simpler sets $\widetilde{\Sigma}_{1}$ and $\widetilde{\Sigma}_{2}$. By Lemmas 10.3 and 10.4 we have that

$$
D u \in W_{\text {loc }}^{\gamma, \frac{\gamma}{2} ; 2\left(1+\sigma_{0}\right)}\left(Q_{T}, \mathbb{R}^{n N}\right), \quad \forall \gamma<\beta .
$$

Therefore applying Proposition 3.3 to $D u$ we conclude that

$$
\operatorname{dim}_{\mathcal{P}}\left(\widetilde{\Sigma}_{1}\right) \leqslant n+2-2 \beta-\delta, \quad \operatorname{dim}_{\mathcal{P}}\left(\widetilde{\Sigma}_{2}\right) \leqslant n+2-2 \beta-\delta,
$$

where this time

$$
\delta:=2 \beta \sigma_{0}
$$

and $\sigma_{0}$ is from Lemma 10.3. The finishes the proof.

As a coda to this paper we report a result that could be interesting in itself since it improves a very classical property concerning the fractional time differentiability of solutions in the case of systems with Hölder continuous coefficients; its statement does not seem to appear elsewhere in the literature.

Proposition 10.5. Let $u \in L^{2}\left(-T, 0 ; W^{1,2}\left(\Omega, \mathbb{R}^{N}\right)\right)$ be a weak solution to the system (1.1) under the assumptions (2.1)-(2.3) and (2.9). Then there exists $\tilde{\delta} \equiv \tilde{\delta}(\beta, L / \lambda) \in(0,1)$ such that

$$
u \in W_{\mathrm{loc}}^{1+\tilde{\delta},(1+\tilde{\delta}) / 2}\left(Q_{T}, \mathbb{R}^{N}\right) .
$$

Proof. The part concerning the higher fractional differentiability in space is a consequence of Lemma 9.5 above, therefore we concentrate on the time derivatives. The idea is to use (9.13) to improve the estimate of the fractional time derivative of $u$ from Lemma 9.1. We only have to modify the argument for the estimate of the term II there. Using (9.13) we find 


$$
\begin{aligned}
|I I| & \leqslant\left(\int_{t_{0}}^{t_{1}} \int_{\Omega} \eta^{2}\left|[a(x, t, u, D u)]_{h}\right|^{2} \mathrm{~d} x \mathrm{~d} t\right)^{1 / 2}\left(\int_{t_{0}}^{t_{1}} \int_{\Omega} \eta^{2}\left|D \tau_{h} u\right|^{2} \mathrm{~d} x \mathrm{~d} t\right)^{1 / 2} \\
& \leqslant L\left(\int_{t_{0}}^{t_{1}} \int_{\Omega} \eta^{2}\left[(1+|D u|)^{2}\right]_{h} \mathrm{~d} x \mathrm{~d} t\right)^{1 / 2}\left(c_{1}|h|^{\beta}+c_{2} h^{\frac{\beta \sigma}{2(1+\sigma)}}\right)^{1 / 2} \\
& \leqslant L\left(\int_{Q_{T}}(1+|D u|)^{2} \mathrm{~d} z\right)^{1 / 2}\left(c_{1}|h|^{\beta}+c_{2}|h|^{\frac{\beta \sigma}{2(1+\sigma)}}\right)^{1 / 2} .
\end{aligned}
$$

Combining the estimates for $I$ and $I I$ with (9.1) and proceeding as in the proof of Lemma 9.1, we obtain that for every open subset $\widetilde{\Omega} \Subset \Omega$ and $\left(t_{0}, t_{1}\right) \Subset(-T, 0)$, there exists a constant $c$ such that

$$
\int_{t_{0}} \int_{\widetilde{\Omega}}^{t_{1}}\left|\tau_{h} u\right|^{2} \mathrm{~d} x \mathrm{~d} t \leqslant c\left(c_{1}|h|^{\beta}+c_{2}|h|^{\frac{\beta \sigma}{2(1+\sigma)}}\right)^{1 / 2}|h|,
$$

yielding the desired improvement for the time derivative of $u$, which is now a consequence of the previous estimate and Proposition 3.4. Indeed, also comparing Lemma 9.5, it comes out that as a number working for the statement we can pick

$$
\tilde{\delta}:=\frac{\beta \sigma}{4(1+\sigma)},
$$

and the dependence announced in the statement follows from the one of $\sigma$ in (9.9).

\section{Acknowledgements}

Part of this paper was written during G.M's stay at the University of Erlangen-Nürnberg as "Von Staudt Professor" in April-May 2004: he wishes to thank people from the Department of Mathematics for the warm hospitality and support. G.M.'s research has been financially supported by MIUR via the project "Calcolo delle Variazioni" (PIN 2002), by INDAM-CNR via the project "Immersioni minimali e mappe p-armoniche" and by the "Otto and Edith Haupt foundation" in Erlangen. Both the authors wish to thank Klaus Steffen for a careful reading of a preliminary draft of the paper and for generous comments leading to nice improvements.

\section{References}

[1] E. Acerbi, G. Mingione, G.A. Seregin, Regularity results for parabolic systems related to a class of non-Newtonian fluids, Ann. Inst. H. Poincaré Anal. Non Linéaire 21 (2004) 25-60.

[2] A.A. Arkhipova, On a partial regularity up to the boundary of weak solutions to quasilinear parabolic systems with quadratic growth, J. Math. Sci. (New York) 101 (2000) 3385-3397 (1997 in Russian).

[3] B. Bojarski, T. Iwaniec, Analytical foundations of the theory of quasiconformal mappings in $R^{n}$, Ann. Acad. Sci. Fenn. Ser. A I 8 (1983) 257-324.

[4] S. Campanato, Equazioni paraboliche del secondo ordine e spazi $\mathfrak{L}^{2, \theta}(\Omega, \delta)$, Ann. Mat. Pura Appl. 73 (4) (1996) 55-102.

[5] S. Campanato, On the nonlinear parabolic systems in divergence form. Hölder continuity and partial Hölder continuity of the solutions, Ann. Mat. Pura Appl. 137 (4) (1984) 83-122.

[6] G. Da Prato, Spazi $\mathcal{L}^{(p, \vartheta)}(\Omega, \delta)$ e loro proprietà, Ann. Mat. Pura Appl. 69 (4) (1965) 383-392.

[7] E. De Giorgi, Frontiere orientate di misura minima, Sem. Scuola Normale Superiore Pisa (1960-1961).

[8] E. De Giorgi, Un esempio di estremali discontinue per un problema variazionale di tipo ellittico, Boll. Un. Mat. Ital. 1 (4) (1968) $135-137$.

[9] E. DiBenedetto, Degenerate Parabolic Equations, Universitext, vol. XV, Springer-Verlag, New York, NY, 1993, p. 387. 
[10] F. Duzaar, A. Gastel, G. Mingione, Elliptic systems, singular sets and Dini continuity, Comm. Partial Differential Equations 29 (2004).

[11] F. Duzaar, J.F. Grotowski, Optimal interior partial regularity for nonlinear elliptic systems: the method of $A$-harmonic approximation, Manuscripta Math. 103 (2000) 267-298.

[12] F. Duzaar, G. Mingione, The $p$-harmonic approximation and the regularity of $p$-harmonic maps, Calc. Var. Partial Differential Equations 20 (2004) 235-256.

[13] F. Duzaar, G. Mingione, Regularity for degenerate elliptic problems via $p$-harmonic approximation, Ann. Inst. H. Poincaré Anal. Non Linéaire 21 (2004).

[14] F. Duzaar, G. Mingione, K. Steffen, Second order parabolic systems with $p$-growth and regularity, in press.

[15] F. Duzaar, K. Steffen, Optimal interior and boundary regularity for almost minimizers to elliptic variational integrals, J. Reine Angew. Math. 546 (2002) 73-138.

[16] M. Giaquinta, Multiple Integrals in the Calculus of Variations and Non Linear Elliptic Systems, Ann. of Math. Stud., vol. 105, Princeton University Press, 1983.

[17] M. Giaquinta, M. Struwe, On the partial regularity of weak solutions of nonlinear parabolic systems, Math. Z. 179 (1982) $437-451$.

[18] M. Giaquinta, M. Struwe, An optimal regularity result for a class of quasilinear parabolic systems, Manuscripta Math. 36 (1981) $223-239$.

[19] E. Giusti, Direct Methods in the Calculus of Variations, World Scientific, Singapore, 2003.

[20] W. Hao, S. Leonardi, J. Necas, An example of irregular solution to a nonlinear Euler-Lagrange elliptic system with real analytic coefficients, Ann. Scuola Norm. Sup. Pisa Cl. Sci. 23 (4) (1996) 57-67.

[21] O. John, J. Stará, Some (new) counterexamples of parabolic systems, Comment. Math. Univ. Carolin. 36 (1995) 503-510.

[22] O. John, J. Stará, On the regularity of weak solutions to parabolic systems in two spatial dimensions, Comm. Partial Differential Equations 23 (1998) 1159-1170.

[23] O. John, J. Stará, On the existence of time derivative of weak solutions to parabolic systems, in: Navier-Stokes Equations. Theory and Numerical Methods, Proceedings of the International Conference, Varenna, 1997, R. Salvi (Ed.), in: Pitman Res. Notes Math. Ser., vol. 388, Longman, Harlow, 1998, pp. 193-200.

[24] J. Kinnunen, J.L. Lewis, Higher integrability for parabolic systems of p-Laplacian type, Duke Math. J. 102 (2000) $253-271$.

[25] A. Koshelev, Regularity Problem for Quasilinear Elliptic and Parabolic Systems, Lecture Notes in Math., vol. 1614, Springer, 1995.

[26] M. Kronz, Partial regularity results for minimizers of quasiconvex functionals of higher order, Ann. Inst. H. Poincaré Anal. Non Linéaire 19 (2002) 81-112.

[27] O.A. Ladyzhenskaya, V.A. Solonnikov, N.N. Ural'tseva, Linear and quasi-linear equations of parabolic type, Transl. Math. Monographs, vol. 23, Amer. Math. Soc., 1968.

[28] J.L. Lions, Quelques methodes des resolution des problemes aux limites non lineaires, Gauthier-Villars, Paris, 1969.

[29] J. Malý, J. Stará, O. John, A note on the regularity of autonomous quasilinear elliptic and parabolic systems, Comm. Partial Differential Equations 13 (1988) 895-903.

[30] G. Mingione, The singular set of solutions to non-differentiable elliptic systems, Arch. Ration. Mech. Anal. 166 (2003) $287-301$.

[31] G. Mingione, Bounds for the singular set of solutions to non linear elliptic systems, Calc. Var. Partial Differential Equations 18 (2003) 373-400.

[32] M. Misawa, Partial regularity results for evolutional $p$-Laplacian systems with natural growth, Manuscripta Math. 109 (2002) $419-454$.

[33] M. Misawa, Local Hölder regularity of gradients for evolutional p-Laplacian systems, Ann. Mat. Pura Appl. 181 (4) (2002) $389-405$.

[34] R. Moser, Regularity for the approximated harmonic map equation and application to the heat flow for harmonic maps, Math. Z. 243 (2003) 263-289.

[35] J. Naumann, J. Wolf, Interior integral estimates on weak solutions of nonlinear parabolic systems, Preprint 94-11, Inst. für Math., Humboldt University, 1994.

[36] J. Naumann, J. Wolf, M. Wolff, On the Hölder continuity of weak solutions to nonlinear parabolic systems in two space dimensions, Comment. Math. Univ. Carolin. 39 (1998) 237-255.

[37] J. Necas, V. Sverák, On regularity of solutions of nonlinear parabolic systems, Ann. Scuola Norm. Super. Pisa Cl. Sci. 18 (4) (1991) 1-11.

[38] G.A. Seregin, Interior regularity for solutions to the modified Navier-Stokes equations, J. Math. Fluid Mech. 1 (1999) $235-281$.

[39] J. Simon, Compact sets in the space $L^{p}(0, T ; B)$, Ann. Mat. Pura Appl. 146 (4) (1987) 65-96.

[40] L. Simon, Lectures on Geometric Measure Theory, Proc. Centre Math. Anal., Austr. Nat. Univ., Canberra, 1983.

[41] L. Simon, Theorems on Regularity and Singularity of Energy Minimizing Maps, Lectures in Math., ETH Zürich, Birkhäuser, Basel, 1996.

[42] J. Stará, O. John, J. Malý, Counterexample to the regularity of weak solution of the quasilinear parabolic system, Comment. Math. Univ. Carolin. 27 (1986) 123-136.

[43] E.W. Stredulinsky, Higher integrability from reverse Hölder inequalities, Indiana Univ. Math. J. 29 (1980) 407-413.

[44] M. Struwe, On the Hölder continuity of bounded weak solutions of quasilinear parabolic systems, Manuscripta Math. 35 (1981) $125-145$.

[45] M. Struwe, A counterexample in regularity theory for parabolic systems, Czech. Math. J. 34 (1984) 183-188.

[46] V. Sverák, X. Yan, Non Lipschitz minimizers of smooth uniformly convex variational integrals, Proc. Nat. Acad. Sci. USA 99 (24) (2002) 15268-15276.

[47] T. Zhong, $C^{1, \alpha}$-partial regularity of nonlinear parabolic systems, J. Partial Differential Equations 5 (1992) 23-34. 